\title{
DYNAMIQUE DES APPLICATIONS RATIONNELLES DES ESPACES MULTIPROJECTIFS
}

\author{
CHARLES FAVRE \& VINCENT GUEDJ
}

\begin{abstract}
We study the dynamics of rational mappings $f$ of $\mathbb{C}^{k}$ by compactifying them in multiprojective spaces $\mathbb{P}^{n_{1}} \times \cdots \times \mathbb{P}^{n_{s}}$. We focus on maps of the surface $\mathbb{P}^{1} \times \mathbb{P}^{1}$. We follow the approach of [Si 99] and associates to any algebraically stable $f$ an invariant positive closed $(1,1)$ current. We then consider the existence of an $f^{*}$ invariant measure using the theory of pluripositive currents, and relates it to the measure of Russsakovskii-Shiffman describing the distribution of preimages of points. Our point of view enables us to treat new classes of examples: we consider in particular polynomial skew products with varying degrees, and birational polynomial mappings of $\mathbb{C}^{2}$. We also describe the compact convex set of $f^{*}$ invariant currents for monomial and birational maps of $\mathbb{C}^{2}$.
\end{abstract}

\section{INTRODUCTION}

Ce travail a pour objet l'étude de l'itération des endomorphismes rationnels $f$ de $\mathbb{C}^{k}$. Il est naturel de considérer l'extension de ces applications à l'espace projectif complexe $\mathbb{P}^{k}$ pour tirer profit de leur algébricité ; c'est le point de vue qui a été adopté par tous les auteurs jusqu'à présent, sous l'impulsion notamment des travaux de Fornaess et Sibony (voir [F$\mathrm{S}$ 94], [F-S 95] et [Si 99]). Un des premiers résultats de la théorie est la construction d'un "courant de Green", c'est un courant positif fermé de bidegré $(1,1)$ dans $\mathbb{P}^{k}$ dont le support concide -dans les bons cas- avec l'ensemble de Julia et qui est invariant par $f$. Il faut cependant supposer que l'application est "algébriquement stable" dans $\mathbb{P}^{k}$, ce qui revient à dire que $\operatorname{deg}\left(f^{j}\right)=(\operatorname{deg} f)^{j}$, où deg $f$ désigne le degré algébrique de $f$.

Il semble difficile d'amorcer une théorie générale des endomorphismes rationnels de $\mathbb{C}^{k}$ qui ne sont pas algébriquement stables dans $\mathbb{P}^{k}$. Nous avons observé qu'il est parfois préférable de considérer l'extension de $f$ dans une compactification $X$ différente de $\mathbb{P}^{k}$. Une compactification de $\mathbb{C}^{k}$ est la donnée d'une variété complexe compacte $X$-que nous supposerons lisse, projective- et d'un diviseur $Y$ de $X$-non nécessairement irréductible- tels que $X \backslash Y$ est biholomorphe à $\mathbb{C}^{k}$ (voir e.g. [P-S 91]). Nous considérons ici le cas d'un espace multiprojectif $X=\mathbb{P}^{n_{1}} \times \cdots \times \mathbb{P}^{n_{s}}$. De nombreux endomorphismes rationnels de $\mathbb{C}^{n_{1}+\cdots+n_{s}}$ ne sont pas algébriquement stables dans $\mathbb{P}^{n_{1}+\cdots+n_{s}}$ mais le sont dans $X=\mathbb{P}^{n_{1}} \times \cdots \times \mathbb{P}^{n_{s}}$ (voir définition 1.8 et proposition 1.9 ci-dessous). Nous construisons pour ces applications un courant de Green et montrons qu'il possède des propriétés similaires à celles

1991 Mathematics Subject Classification. Primary: 32H50, Secondary: 32H04, 32C30.

Key words and phrases. complex dynamics, multiprojective spaces, skew products, birational mappings, pluripositive currents. 
des courants de Green d'applications algébriquement stables de $\mathbb{P}^{k}$; nous nous appuyons de façon essentielle sur le récent survey de Sibony [Si 98].

Il est notable que la plupart des endomorphismes rationnels $f$ de $\mathbb{C}^{k}$ que nous considérons ne sont pas algébriquement stables dans $\mathbb{P}^{k}$, ni même birationnellement conjugués à des applications algébriquement stables de $\mathbb{P}^{k}$. Le premier degré dynamique $\lambda_{1}(f)$ (voir définition 4.1 et lemme 4.4) constitue en effet un invariant par conjugaison birationnelle qui est nécessairement entier si $f$ est algébriquement stable dans $\mathbb{P}^{k}$; nous étudions des applications dont le premier degré dynamique est dans $\frac{1}{2} \mathbb{Z}[\sqrt{k}]$ avec $k \in \mathbb{N}$.

Un de nos objectifs était de tenter de préciser la nature de la mesure limite $\mu_{f}$ construite par Russakovskii-Shiffman [R-S 97] qui rend compte de l'équidistribution des points par image inverse (voir théorème 4.6). Nous proposons une construction alternative qui permet d'exprimer $\mu_{f}$ comme la courbure d'un courant "pluripositif" (au sens de [Si 85]) qui ne charge pas les ensembles pluripolaires. Cela nous permet de montrer que $\mu_{f}$ ne charge pas -en général- l'ensemble de normalité (théorème 4.15) et qu'elle est mélangeante dans le cas des produits croisés (section 6). Notons que la construction de courants pluripositifs intervient également dans [G-S 99] pour définir certaines mesures mélangeantes pour des automorphismes polynomiaux de $\mathbb{C}^{k}$.

Par commodité, nous nous sommes restreints au cas où $X$ ne comporte que deux facteurs et, très souvent, au cas $X=\mathbb{P}^{1} \times \mathbb{P}^{1}$. Nous avons en effet préféré insister sur les nouvelles familles d'exemples que l'on peut traiter ainsi, ce qui jusitifie l'importance relative des deux dernières sections.

Détaillons à présent le contenu de l'article.

Après avoir brièvement rappeler la forme des endomorphismes rationnels $f$ des espaces multiprojectifs, nous introduisons dans la section 1 les notions dynamiques fondamentales pour la suite: ensemble d'indétermination, applications algébriquement stables, ensembles de Fatou, Julia et de normalité.

Nous construisons le courant de Green $T_{f}$ de $f$ dans la section 2 (théorème 2.2). C'est la limite de $K^{-j}\left(f^{j}\right)^{*}(\omega)$ où $\omega$ est une forme de Kähler normalisée et $K$ est la plus grande valeur propre de la matrice des degrés de $f$. Le courant $T_{f}$ est à support dans l'ensemble de Julia -avec égalité si $f$ est normale (corollaire 2.7)- et ne charge pas les hypersurfaces (théorème 2.8). Il est extrémal dans le convexe compact $\mathcal{K}_{f}$ des courants positifs fermés $S$ de bidegré $(1,1)$ sur $X$ tels que $f^{*} S=K \cdot S$ (proposition 2.4).

Dans la section 3 nous explicitons par des arguments d'estimées volumiques les éléments extrémaux du convexe $\mathcal{K}_{f}$ lorsque $f=\left(z^{\alpha} w^{\beta}, z^{\gamma} w^{\delta}\right)$ est une application monomiale de $\mathbb{C}^{2}$ (théorème 3.1 ). Ces estimations serviront également à établir des résultats de convergence dans la section 7 .

Nous rappelons dans la section 4 la définition des degrés dynamiques $\lambda_{l}(f)$ due à Friedland [F 91]. Nous transportons dans $\mathbb{P}^{1} \times \mathbb{P}^{1}$ le résultat de Russakovskii-Shiffman [R-S 97] qui assure, sous l'hypothèse $\lambda_{2}(f)>\lambda_{1}(f)$, l'existence d'une mesure de probabilité $\mu_{f}$ et d'un ensemble pluripolaire $\mathcal{E}_{f}$ dans $\mathbb{P}^{1} \times \mathbb{P}^{1}$ tels que

$$
\text { (E) } \frac{1}{\lambda_{2}\left(f^{j}\right)}\left(f^{j}\right)^{*}\left(\varepsilon_{a}\right) \longrightarrow \mu_{f}, \forall a \in \mathbb{P}^{1} \times \mathbb{P}^{1} \backslash \mathcal{E}_{f},
$$


où $\varepsilon_{a}$ désigne la masse de Dirac au point $a$. Lorsque $\mu_{f}$ ne charge pas les ensembles pluripolaires, $(E)$ implique que $\mu_{f}$ est mélangeante pour $f$ (lemme 4.11). Nous montrons que $\mu_{f}$ admet un potentiel continu pluripositif dans l'ensemble de normalité et nous établissons des inégalités de Chern-LevineNirenberg pour ces courants afin de montrer que $\mu_{f}$ ne charge pas certains ensembles pluripolaires. Cette technique sera appliquée notamment aux produits croisés (section 6).

La section 5 traite le cas des applications polynomiales de $\mathbb{C}^{2}$ qui admettent une extension méromorphe algébriquement stable dans $\mathbb{P}^{1} \times \mathbb{P}^{1}$. Lorsque l'application n'est pas un produit croisé, la fonction de Green (potentiel de $T_{f}$ ) est continue et positive dans $\mathbb{C}^{2}$, c'est la fonction d'échappement vers le point $(\infty, \infty)$ qui est superattractif (théorème 5.2). Nous exhibons des exemples d'applications polynomiales non normales de $\mathbb{C}^{2}$ pour lesquelles un disque holomorphe est isolé dans leur ensemble de Julia.

Nous considérons dans la section 6 le cas des "produits croisés" polynomiaux de $\mathbb{C}^{2}, f=(P(z), Q(z, w))$. Lorsque $f$ n'est pas semi-linéaire, nous montrons que la mesure limite $\mu_{f}$ est mélangeante, qu'elle admet des exposants de Lyapunov strictement positifs et nous précisons certains aspects de la dynamique. La fonction de Green peut être discontinue. Nous obtenons les premiers exemples explicites d'applications polynomiales de $\mathbb{C}^{2}$ dont l'ensemble des points d'orbite positive bornée n'est pas fermé et l'ensemble récurrent non borné dans $\mathbb{C}^{2}$. Des applications similaires ont été étudiées par différents auteurs, nous nous sommes inspirés de Jonsson [Jo 99] qui considère le cas des produits croisés polynomiaux de $\mathbb{C}^{2}$ admettant une extension holomorphe dans $\mathbb{P}^{2}$.

La section 7 est consacrée à la dynamique des applications birationnelles algébriquement stables dans $\mathbb{P}^{2}$ ou $\mathbb{P}^{1} \times \mathbb{P}^{1}$. Nous caractérisons tout d'abord tous les courants $T$ positifs fermés de bidegré $(1,1)$ t.q. $\quad f^{*} T=\lambda_{1}(f) T$. Etant donné $S$ un courant positif fermé de bidegré $(1,1)$, nous donnons une condition optimale sur les nombres de Lelong de $S$ pour que la suite de courants $\lambda_{1}(f)^{-j}\left(f^{j}\right)^{*} S$ converge vers $T_{f}$ (théorème 7.6). La démonstration s'appuie sur des estimées volumiques fines. Elle reprend les idées développées par Bedford-Smillie ([B-S 91],[B-S 92]), Fornaess-Sibony ([F-S 92],[Si 98]) et Diller [Di 96] qui obtiennent des résultats plus faibles de convergence. Enfin nous étudions précisément la dynamique d'une classe $\mathcal{H}$ d'applications birationnelles polynomiales de $\mathbb{C}^{2}$. Nous conjecturons que toute application birationnelle polynomiale $f$ de $\mathbb{C}^{2}$ avec $\lambda_{1}(f)>1$ est conjuguée à un élément de cette classe. Toute application de $\mathcal{H}$ se compactifie de manière algébriquement stable soit dans $\mathbb{P}^{2}$, soit dans $\mathbb{P}^{1} \times \mathbb{P}^{1}$, et nous construisons une mesure mélangeante qui lui est naturellement associée.

\section{Notations, DÉFInitions}

Dans toute la suite, $X$ désignera un espace multiprojectif complexe, i.e. un produit d'espaces projectifs complexes. Pour simplifier les notations, nous nous limiterons au cas d'un produit de deux espaces $X=\mathbb{P}^{n} \times \mathbb{P}^{m}$. Un point $x$ est défini par ses coordonnées homogènes $([z],[w])$ où $[z]=\left[z_{0}\right.$ : $\left.\cdots: z_{n}\right]$ et $[w]=\left[w_{0}: \cdots: w_{m}\right]$. Une application méromorphe $f: X \rightarrow X$ 
est nécessairement rationnelle puisque $X$ est projective et s'écrit alors

$$
f=\left(\left[P_{0}: \cdots: P_{n}\right],\left[Q_{0}: \cdots: Q_{m}\right]\right),
$$

où les $P_{i}$ sont des polynômes bihomogènes de bidegré $(\alpha, \beta)$ en $(z, w)$ sans facteur commun, et les $Q_{j}$ sont des polynômes bihomogènes de bidegré $(\gamma, \delta)$ sans facteur commun également. On notera

$$
A_{f}=A:=\left[\begin{array}{ll}
\alpha & \gamma \\
\beta & \delta
\end{array}\right]
$$

la matrice des degrés de $f$. On associe à $f$ une application $F=(P, Q)$ de $\mathbb{C}^{n+1} \times \mathbb{C}^{m+1}$ dans $\mathbb{C}^{n+1} \times \mathbb{C}^{m+1}$. Si

$$
\pi:\left(\mathbb{C}^{n+1} \backslash\{0\}\right) \times\left(\mathbb{C}^{m+1} \backslash\{0\}\right) \rightarrow X
$$

est la projection canonique, on a la propriété de commutation $\pi \circ F=f \circ \pi$. On notera $J(F)$ le jacobien de $F$.

La matrice $A$ étant fixée, l'espace des applications polynomiales $F=$ $(P, Q)=\left(P_{0}, \ldots, P_{n}, Q_{0}, \ldots, Q_{m}\right)$ où les $P_{i}$ (resp. $\left.Q_{j}\right)$ sont des polynômes bihomogènes de bidegré $(\alpha, \beta)$ (resp. $(\gamma, \delta))$ en $(z, w)$ s'identifie à $\mathbb{C}^{N_{A}+1} \times$ $\mathbb{C}^{M_{A}+1}$ avec

$$
\begin{aligned}
& N_{A}=(n+1)\left(\begin{array}{c}
\alpha+n \\
n
\end{array}\right)\left(\begin{array}{c}
\beta+m \\
m
\end{array}\right)-1, \\
& M_{A}=(m+1)\left(\begin{array}{c}
\gamma+n \\
n
\end{array}\right)\left(\begin{array}{c}
\delta+m \\
m
\end{array}\right)-1 .
\end{aligned}
$$

La condition sur les $P_{i}$ (resp. sur les $Q_{j}$ ) de ne pas avoir de facteurs communs s'exprime comme la non annulation de leur résultant. L'espace Rat $_{A}$ des applications méromorphes de $X$ dans lui-même de degré exactement $A$ s'identifie donc canoniquement à un ouvert de Zariski de $\mathbb{P}^{N_{A}} \times \mathbb{P}^{M_{A}}$.

Définition 1.1. On notera $\mathcal{M}_{A}$ le sous ensemble de $\mathbb{P}^{N_{A}} \times \mathbb{P}^{M_{A}}$ constitué des applications rationnelles de $X$ dans lui même de degrés A qui sont dominantes i.e. génériquement de rang $n+m$.

Proposition 1.2. L'ensemble $\mathcal{M}_{A}$ est vide uniquement dans l'un des cas suivants:

- $\alpha=\gamma=0$,

- $\beta=\delta=0$,

- $\alpha=0$ et $n>m$,

- $\delta=0$ et $n<m$.

On notera $\mathcal{M}_{2}(\mathbb{N})$ l'ensemble des matrices $2 \times 2$ à coefficients dans $\mathbb{N}$ telles qu'aucun de ces cas n'ait lieu. On a $A, B \in \mathcal{M}_{2}(\mathbb{N}) \Rightarrow A \cdot B \in \mathcal{M}_{2}(\mathbb{N})$.

Si $A \in \mathcal{M}_{2}(\mathbb{N})$, l'ensemble $\mathcal{M}_{A}$ est un ouvert de Zariski dense de $\mathbb{P}^{N_{A}} \times$ $\mathbb{P}^{M_{A}}$. En particulier $\mathcal{M}_{A}$ est connexe.

Démonstration. La preuve de la première assertion est élémentaire. Il

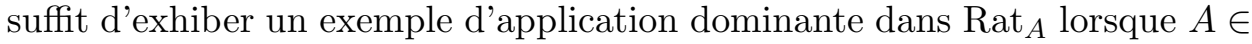
$\mathcal{M}_{2}(\mathbb{N})$ (voir exemple 1.12 à la fin de cette section). Le reste est une conséquence élémentaire du théorème de projection propre de Remmert (voir proposition I.1.1 dans [Si 99] qui traite le cas $X=\mathbb{P}^{k}$ ). 
Etant donnée $f \in \mathcal{M}_{A}$, on note $\mathcal{C}_{f}$ son ensemble critique, le sous-ensemble analytique propre de $X$ défini par l'annulation du jacobien de $f$ dans un système de coordonnées locales.

Proposition 1.3. Soit $A \in \mathcal{M}_{2}(\mathbb{N})$ et $f \in \mathcal{M}_{A}$.

L'ensemble critique $\mathcal{C}_{f}$ est une hypersurface de $X$ de bidegré

$$
((n+1)(\alpha-1)+(m+1) \gamma,(n+1) \beta+(m+1)(\delta-1)) .
$$

Si $\operatorname{det} A \neq 0, \mathcal{C}_{f}$ est donné par

$$
\mathcal{C}_{f}=\left\{([z],[w]) \in \mathbb{P}^{n} \times \mathbb{P}^{m} / J(F)(z, w)=0\right\} .
$$

Démonstration. Par 1.2, l'ouvert de Zariski $\mathcal{M}_{A}$ est connexe donc le bidegré de $J(f)$ qui dépend continument de $f$ est constant dans $\mathcal{M}_{A}$. Il suffit de le calculer sur des applications monomiales pour obtenir la valeur annoncée (voir exemple 1.12).

Considérons l'application monomiale de $\mathbb{C}^{2}$ dans lui-même définie par $H(x, y):=\left(x^{\alpha} y^{\beta}, x^{\gamma} y^{\delta}\right)$. Fixons $p_{0} \in X$ et des trivialisations locale de $\pi$ dans des voisinages $U$ de $p_{0}$ et $V$ de $f\left(p_{0}\right)$. L'application $F$ se réécrit dans ces cartes $F: U \times\left(\mathbb{C}^{*}\right)^{2} \rightarrow V \times\left(\mathbb{C}^{*}\right)^{2}$ sous la forme $F(p, x, y)=$ $(f(p), \psi(p) H(x, y))$ où $\psi$ est une fonction holomorphe dans $U$ qui ne s'annule pas. On en déduit que pour tout $(x, y)$ fixé, $J(F)(p, x, y) / J(f)(p)=\psi(p)$. $J_{H}(x, y)$ est une fonction qui ne s'annule pas dans $U$, ce qui donne l'égalité $\mathcal{C}_{f}=\left\{([z],[w]) \in \mathbb{P}^{n} \times \mathbb{P}^{m} / J(F)(z, w)=0\right\}$.

La cohomologie des espaces multiprojectifs est très simple à décrire. Le second groupe de de Rham à coefficients dans $\mathbb{R}$ est égal a $H^{1,1}(X, \mathbb{R})$ qui, dans le cas d'un biproduit, est isomorphe à $\mathbb{R}^{2}$. En particulier, tout courant positif fermé $T$ de bidegré $(1,1)$ sur $X$ est cohomologue à $a \omega_{1}+b \omega_{2}$ avec $a=\int_{X} T \wedge \omega_{1}^{n-1} \wedge \omega_{2}^{m}$ et $b=\int_{X} T \wedge \omega_{1}^{n} \wedge \omega_{2}^{m-1}$, où $\omega_{1}$ (resp. $\omega_{2}$ ) désigne le pull-back de la forme de Fubini-Study de $\mathbb{P}^{n}$ (resp. $\mathbb{P}^{m}$ ) par la projection sur le premier (resp. second) facteur. Notons que $\omega_{1}^{j} \equiv 0$ si $j \geq n+1$ (resp. $\omega_{2}^{j} \equiv 0$ si $\left.j \geq m+1\right)$ et $\int_{X} \omega_{1}^{n} \wedge \omega_{2}^{m}=1$.

On notera $\omega:=\omega_{1}+\omega_{2}$ la forme de Kähler sur $X$ par rapport à laquelle nous calculerons les masses des courants positifs dans la suite de cet article.

Définition 1.4. Soit $A \in \mathcal{M}_{2}(\mathbb{N})$ et $f=([P],[Q]) \in \mathcal{M}_{A}$. L'ensemble d'indétermination de $f$ est le sous-ensemble analytique de codimension au moins 2 défini par $I_{f}=I_{P} \cup I_{Q}$, où

$$
\begin{aligned}
& I_{P}=\left\{([z] ;[w]) \in X \text { t.q. } P_{i}(z, w)=0 \text { pour } i=0, \cdots, n\right\}, \\
& I_{Q}=\left\{([z] ;[w]) \in X \text { t.q. } Q_{j}(z, w)=0 \text { pour } j=0, \cdots, m\right\} .
\end{aligned}
$$

On notera $E_{f}:=\overline{\cup_{k \in \mathbb{N}} I_{f^{k}}}$ l'adhérence de l'ensemble des points d'indétermination des itérés de $f$.

\section{Proposition 1.5.}

(1) Soit $A \in \mathcal{M}_{2}(\mathbb{N})$ et $f \in \mathcal{M}_{A}$. Alors $I_{P}$ est non vide sauf si $\beta=0$ et $[z] \rightarrow[P(z)]$ est holomorphe dans $\mathbb{P}^{n}$ ou si $\alpha=0, m=n$ et $[w] \rightarrow[P(w)]$ est holomorphe dans $\mathbb{P}^{n}$.

(2) Soit $A \in \mathcal{M}_{2}(\mathbb{N})$. L'ensemble $\left\{f \in \mathcal{M}_{A}\right.$ t.q. $\left.I_{P} \cap I_{Q}=\emptyset\right\}$ est un ouvert de Zariski dense de $\mathbb{P}^{N_{A}} \times \mathbb{P}^{M_{A}}$. 


\section{Démonstration.}

1. L'ensemble $I_{P}$ est l'intersection des $n+1$ hypersurfaces $\left\{P_{j}=0\right\}$. Elles sont toutes de bidegré $(\alpha, \beta)$, donc le courant d'intégration sur $\left\{P_{j}=0\right\}$, que l'on note $\left[P_{j}=0\right]$ est cohomologue à $\alpha \omega_{1}+\beta \omega_{2}$. Si $I_{P}$ est vide le courant $S:=\left[P_{0}=0\right] \wedge \ldots \wedge\left[P_{n}=0\right]$ est bien défini, identiquement nul, et cohomologue à $\left(\alpha \omega_{1}+\beta \omega_{2}\right)^{n+1}$ donc

$$
0=\int_{X} S \wedge \omega_{2}^{m-1}=\int_{X}\left(\alpha \omega_{1}+\beta \omega_{2}\right)^{n+1} \wedge \omega_{2}^{m-1}=n \alpha^{n} \beta .
$$

Si $\beta=0, P_{j}$ est alors indépendant de $w$ et $I_{P}$ est vide ssi $[z] \in \mathbb{P}^{n} \rightarrow[P(z)] \in$ $\mathbb{P}^{n}$ est holomorphe. Si $\alpha=0$, nécessairement $m \geq n$ car $f$ est dominante. Or l'intersection de $n+1$ hypersurfaces de $\mathbb{P}^{m}$ ne peut être vide que si $m \leq n$ (théorème de Bezout), donc $m=n$ et $[w] \in \mathbb{P}^{n} \rightarrow[P(w)] \in \mathbb{P}^{n}$ doit être holomorphe.

2. Soit $\Sigma^{\prime}=\left\{(f, x) \in\left(\mathbb{P}^{N_{A}} \times \mathbb{P}^{M_{A}}\right) \times X\right.$ t.q. $\left.P(x)=Q(x)=0\right\}$ et $\Sigma=p\left(\Sigma^{\prime}\right)$ où $p:\left(\mathbb{P}^{N_{A}} \times \mathbb{P}^{M_{A}}\right) \times X \rightarrow \mathbb{P}^{N_{A}} \times \mathbb{P}^{M_{A}}$ désigne la projection holomorphe propre sur le premier facteur. Le théorème de Remmert assure que $\Sigma$ est un sous-ensemble analytique de $\mathbb{P}^{N_{A}} \times \mathbb{P}^{M_{A}}$. L'ensemble $\left\{f \in \mathcal{M}_{A} / I_{P} \cap I_{Q}=\emptyset\right\}=\mathcal{M}_{A} \backslash \Sigma$ est donc un ouvert de Zariski de $\mathbb{P}^{N_{A}} \times \mathbb{P}^{M_{A}}$ qui n'est pas vide comme le montre l'exemple 1.12.

Remarque 1.6. Il résulte de i) que les seules applications holomorphes dominantes de $\mathbb{P}^{n} \times \mathbb{P}^{m}$ dans lui même sont du type $([z] ;[w]) \mapsto([P(z)] ;[Q(w)])$ avec $P$ et $Q$ holomorphes, ou du type $([z],[w]) \mapsto([P(w)] ;[Q(z)])$ avec $P$ et $Q$ holomorphes, lorsque de plus $n=m$.

Si $A, B \in M_{2}(\mathbb{N})$, on notera $A \leq B$ si tous les coefficients de $A$ sont plus petits que ceux de $B$.

Proposition 1.7. Soit $A, B \in \mathcal{M}_{2}(\mathbb{N}), f \in \mathcal{M}_{A}$ et $g \in \mathcal{M}_{B}$.

Alors $f \circ g \in \mathcal{M}_{C}$, où $C \leq B \cdot A$ avec égalité ssi il n'existe pas d'hypersurface $V$ de $X$ t.q. $g\left(V \backslash I_{g}\right) \subset I_{f}$.

Démonstration. Il s'agit d'une adaptation immédiate du cas $X=\mathbb{P}^{k}$ (voir [F-S 95]).

Définition 1.8. Soit $A \in \mathcal{M}_{2}(\mathbb{N})$. On dit que $f \in \mathcal{M}_{A}$ est algébriquement stable s'il n'existe pas d'entier $k \geq 1$ ni d'hypersurface $V$ tels que $f^{k}(V \backslash$ $\left.I_{f^{k}}\right) \subset I_{f}$.

Il résulte de la proposition précédente que $f$ est algébriquement stable ssi pour tout $k \in \mathbb{N}, f^{k} \in \mathcal{M}_{A^{k}}$. Si $F$ est un relèvement de $f$ dans $\mathbb{C}^{n+1} \times \mathbb{C}^{m+1}$, alors $F^{k}=\left(P_{k} ; Q_{k}\right)$ est un relèvement de $f^{k}$. On démontre comme dans [Si 99] (proposition I.4.5) la

Proposition 1.9. Les applications non algébriquement stables de $\mathcal{M}_{A}$ sont contenues dans une réunion dénombrable de sous-ensembles analytiques propres de $\mathcal{M}_{A}$.

Définition 1.10. Soit $f \in \mathcal{M}_{A}$ une application algébriquement stable. 
- Un point $x$ est dans $\mathcal{F}(f)$ l'ensemble de Fatou de $f$ s'il existe un voisinage $U$ de $x$ tel que la famille $\left(\left.f\right|_{U}\right)^{k}$ soit équicontinue. L'ensemble de Julia, noté $J(f)$, est le complémentaire de l'ensemble de Fatou.

- Un point est dit normal s'il existe un voisinage $U$ de $x$ et un voisinage $V$ de $I_{f}$ tels qu'on ait $f^{j}(U) \cap V=\emptyset$ pour tout $j \in \mathbb{N}$. On note $\mathcal{N}_{f}$ l'ensemble des points normaux de $f$.

- Enfin $f$ est normale si $\mathcal{N}_{f}=X \backslash E_{f}$.

Terminons cette section introductive par quelques exemples.

\section{Exemples 1.11.}

1. Soit $f:\left(z_{1} ; w_{1}\right) \in \mathbb{C} \times \mathbb{C} \rightarrow\left(U\left(z_{1}, w_{1}\right) ; V\left(z_{1}, w_{1}\right)\right) \in \mathbb{C} \times \mathbb{C}$ où $U$ et $V$ sont des polynômes tels que $\operatorname{deg}_{z_{1}} U=\alpha, \operatorname{deg}_{w_{1}} U=\beta, \operatorname{deg}_{z_{1}} V=\gamma$ et $\operatorname{deg}_{w_{1}} V=\delta$. Alors $f$ s'étend en une application rationnelle $\widetilde{f}$ de $\mathbb{P}^{1} \times \mathbb{P}^{1}$,

$$
\widetilde{f}=\left(\left[z_{0}^{\alpha} w_{0}^{\beta}: z_{0}^{\alpha} w_{0}^{\beta} U\left(z_{1} / z_{0}, w_{1} / w_{0}\right)\right],\left[z_{0}^{\gamma} w_{0}^{\delta}: z_{0}^{\gamma} w_{0}^{\delta} V\left(z_{1} / z_{0}, w_{1} / w_{0}\right)\right]\right)
$$

dont la matrice de degrés est

$$
A=\left[\begin{array}{ll}
\alpha & \gamma \\
\beta & \delta
\end{array}\right]
$$

On vérifie aisément que $\widetilde{f}$ est algébriquement stable ssi le degré (total) de $U$ est égal à $\alpha+\beta$ et celui de $V$ est égal à $\gamma+\delta$.

2. L'éclatement de $\mathbb{C}^{2}$ en $0: f(z, w)=(w, z w)$ s'étend en

$$
\widetilde{f}([z],[w])=\left(\left[w_{0}, w_{1}\right],\left[z_{0} w_{0}, z_{1} w_{1}\right]\right) .
$$

On a $I_{P}=\emptyset, I_{\widetilde{f}}=I_{Q}=\{([0,1],[1,0]) ;([1,0],[0,1])\}$. Les seules courbes contractées par $\widetilde{f}$ sont $\left\{w_{0}=0\right\},\left\{w_{1}=0\right\},\left\{z_{0}=0\right\}$ et $\left\{z_{1}=0\right\}$; elles sont envoyées sur des points fixes qui ne sont pas d'indétermination, donc $\widetilde{f}$ est algébriquement stable et on vérifie que $E_{\widetilde{f}}=I_{\widetilde{f}}$. On vérifie que $\tilde{f}$ est normale dans $\mathbb{P}^{1} \times \mathbb{P}^{1}$ (voir section 3). Notons que l'on peut également considérer l'extension méromorphe de $f$ dans $\mathbb{P}^{2}$, mais l'extension obtenue ne sera pas algébriquement stable!

3. Une application de Hénon $f\left(z_{1}, w_{1}\right) \in \mathbb{C}^{2} \rightarrow\left(w_{1}, a z_{1}+w_{1}^{d}\right) \in \mathbb{C}^{2}$ s'étend méromorphiquement à $\mathbb{P}^{1} \times \mathbb{P}^{1}$ par

$$
\widetilde{f}([z],[w])=\left(\left[w_{0}, w_{1}\right],\left[z_{0} w_{0}^{d}, a z_{1} w_{0}^{d}+z_{0} w_{1}^{d}\right]\right) .
$$

On a $A_{f}=\left[\begin{array}{ll}0 & 1 \\ 1 & d\end{array}\right], I_{P}=\emptyset, I_{f}=I_{Q}=\{([0,1],[0,1])\}$. L'application $\tilde{f}$ contracte l'hypersurface $\left\{w_{0}=0\right\}$ sur le point $([0,1],[0,1]) \in I_{\widetilde{f}}$ donc $\widetilde{f}$ n'est pas algébriquement stable.

Exemples 1.12. Soit $a, a^{\prime}, b, b^{\prime} \in \mathbb{C}$ et considérons l'application rationnelle $\widetilde{f}$, extension à $\mathbb{P}^{1} \times \mathbb{P}^{1}$ de l'application polynomiale:

$$
f(z, w)=\left((z-a)^{\alpha}(w-b)^{\beta},\left(z-a^{\prime}\right)^{\gamma}\left(w-b^{\prime}\right)^{\delta}\right) .
$$

La matrice des degrés de $\widetilde{f}$ est donnée par

$$
A_{\tilde{f}}=\left[\begin{array}{ll}
\alpha & \gamma \\
\beta & \delta
\end{array}\right] \text {. }
$$


On vérifie aisément les propriétés suivantes:

(1) $\tilde{f}$ est toujours algébriquement stable;

(2) $I_{P} \cap I_{Q}=\emptyset$ dès que $b \neq b^{\prime}$ et $a \neq a^{\prime}$;

(3) $\widetilde{f}$ est dominante dès que $\alpha \beta \neq \gamma \delta$, ou $\alpha \delta a^{\prime} b \neq \beta \gamma b^{\prime} a$, ou $b^{\prime} \beta \gamma \neq b \alpha \delta$, ou $a^{\prime} \alpha \delta \neq a \beta \gamma$.

\section{Courant de Green}

Soit $A \in \mathcal{M}_{2}(\mathbb{N})$ et $f \in \mathcal{M}_{A}$ une application méromorphe dominante dans $X=\mathbb{P}^{n} \times \mathbb{P}^{m}$. Nous allons lui associer un courant positif fermé $T=T_{f}$ de bidegré $(1,1)$ dans $X$ dont les propriétés sont liées à la dynamique de $f$. On note $\mathcal{T}(X)$ le cône des courants positifs fermés de bidegré $(1,1)$ sur $X$.

Rappelons que si $S \in \mathcal{T}(X)$ est cohomologue à $a \omega_{1}+b$ inputdeuxioomega $a_{2}$, il existe une unique fonction plurisousharmonique $\varphi \in$ $P S H\left(\mathbb{C}^{n+1} \times \mathbb{C}^{m+1}\right)$ telle que

$$
\varphi(\lambda z, \mu w)=a \log |\lambda|+b \log |\mu|+\varphi(z, w), \forall(\lambda, \mu) \in \mathbb{C}^{*} \times \mathbb{C}^{*},
$$

avec $\pi^{*} S=d d^{c} \varphi$ et $\sup _{|z|=|w|=1} \varphi=0$; réciproquement la donnée d'une telle fonction $\varphi$ définit de manière unique un courant $S=\mathcal{L}(\varphi) \in \mathcal{T}(X)$ (voir [G 97] pour une démonstration et des propriétés analogues de représentation des courants sur les variétés homogènes du groupe linéaire). On appelera potentiel de $T \in \mathcal{T}(X)$ toute fonction $\mathcal{L}^{-1}(T)+c, c \in \mathbb{R}$. Avec ces notations, on a $\omega_{1}=\mathcal{L}(\log |z|)$ et $\omega_{2}=\mathcal{L}(\log |w|)$. La convergence faible des courants est alors équivalente à la convergence de leurs potentiels dans $L_{l o c}^{1}$.

Soit $F=(P, Q)$ un relèvement de $f$ dans $\mathbb{C}^{n+1} \times \mathbb{C}^{m+1}$ normalisé de telle sorte que $\sup _{|z|=|w|=1}|P|=\sup _{|z|=|w|=1}|Q|=1$ et soit $S=\mathcal{L}(\varphi) \in \mathcal{T}(X)$. On peut définir $f^{*}(S):=\mathcal{L}(\varphi \circ F)$. Comme $f$ est dominante, l'image d'un ouvert n'est pas contenue dans un sous-ensemble analytique et l'opération $S \rightarrow f^{*}(S)$ est continue pour la topologie des courants.

Dans toute la suite, $f$ sera de bidegrés $(\alpha, \gamma)$ et $(\beta, \delta)$, et on notera $A$ la matrice des degrés de $f$. Le réel $\rho_{+}$désignera la plus grande valeur propre de $A$ et $\rho_{-}$la plus petite. On a donc

$$
\rho_{+}=\frac{\alpha+\delta+\sqrt{(\alpha-\delta)^{2}+4 \beta \gamma}}{2} \text { et } \rho_{-}=\frac{\alpha+\delta-\sqrt{(\alpha-\delta)^{2}+4 \beta \gamma}}{2} .
$$

Lemme 2.1. Soit $A \in \mathcal{M}_{2}(\mathbb{N})$ avec $\beta \gamma \neq 0$. Alors

i) $\rho_{+}>1$ sauf si $\alpha=\delta=0$ et $\beta=\gamma=1$;

ii) $\rho_{+}>\left|\rho_{-}\right|$sauf si $\alpha=\delta=0$;

iii) il existe un unique vecteur propre $(a, b)$ de A tel que $a>0, b>0$ et $a+b=1$. C'est un vecteur propre associé à $\rho_{+}$et c'est l'unique vecteur propre de $A$ à coordonnées non-négatives -normalisé par a + $b=1$;

iv) il existe $c>1$ telle que pour tout $v \in \mathbb{R}^{+} \times \mathbb{R}^{+}$on ait

$$
c^{-1} \rho_{+}^{j}\|v\| \leq\left\|A^{j} \cdot v\right\| \leq c \rho_{+}^{j}\|v\| .
$$

Démonstration. Immédiate. On vérifiera que $a=\gamma /\left(\gamma+\rho_{+}-\alpha\right)$ et $b=\left(\rho_{+}-\alpha\right) /\left(\gamma+\rho_{+}-\alpha\right)$. 
Nous ne perdons rien à supposer $\rho_{+}>1$ (l'égalité correspond à des applications linéaires de $X$ dont la dynamique est triviale). Le cas particulier des produits croisés (i.e. $\beta \gamma=0$ ) sera traité à la section 6 .

Lorsque $f$ est algébriquement stable, il résulte de 1.6 et 2.1.iv) que la masse de $\left(f^{j}\right)^{*} \omega / \rho_{+}^{j}$ est bornée inférieurement et supérieurement.

Théorème 2.2. Soit $A \in \mathcal{M}_{2}(\mathbb{N})$ avec $\beta \gamma \neq 0$ et $\rho_{+}>1$. Soit $\omega^{\prime}:=a \omega_{1}+$ $b \omega_{2}$, où $(a, b)$ l'unique vecteur propre positif de $A$ normalisé par $a+b=1$ (i.e. $\left.\left\|\omega^{\prime}\right\|:=\int_{X} \omega^{\prime} \wedge \omega^{n+m-1}=1\right)$. Soit $f \in \mathcal{M}_{A}$ une application méromorphe dominante algébriquement stable de $\mathbb{P}^{n} \times \mathbb{P}^{m}$.

La suite de courants $\rho_{+}^{-j}\left(f^{j}\right)^{*}\left(\omega^{\prime}\right)$ converge vers un courant positif fermé $T$ de bidegré $(1,1)$ cohomologue à $\omega^{\prime}$ qui vérifie

$$
f^{*}(T)=\rho_{+} \cdot T
$$

Si $F$ est un relèvement de $f, T$ admet un potentiel $G$ qui vérifie

$$
\begin{aligned}
G(\lambda z, \mu w) & =a \log |\lambda|+b \log |\mu|+G(z, w), \\
G(F(z, w)) & =\rho_{+} \cdot G(z, w) .
\end{aligned}
$$

De plus toute fonction $v$ vérifiant ces deux propriétés est telle que $v \leq G$.

Démonstration. Soit $F=(P, Q)$ un relèvement de $f$; les applications $P$ et $Q$ étant définies à une constante multiplicative près, on peut choisir ces constantes de telle sorte que

$$
|P(z, w)| \leq|z|^{\alpha}|w|^{\beta} \text { et }|Q(z, w)| \leq|z|^{\gamma}|w|^{\delta},
$$

d'où en posant $F^{j}=\left(P_{j}, Q_{j}\right)$,

$$
\left|P_{j+1}\right|=\left|P\left(P_{j}, Q_{j}\right)\right| \leq\left|P_{j}\right|^{\alpha}\left|Q_{j}\right|^{\beta} \text { et }\left|Q_{j+1}\right| \leq\left|P_{j}\right|^{\gamma}\left|Q_{j}\right|^{\delta} .
$$

Posons $G_{j}=\rho_{+}^{-j}\left[a \log \left|P_{j}\right|+b \log \left|Q_{j}\right|\right]$; les inégalités précédentes assurent, puisque $A \cdot{ }^{t}(a, b)=\rho_{+} \cdot{ }^{t}(a, b)$, que $G_{j}$ est une suite décroissante de fonctions psh. Donc $G_{j}$ converge vers une fonction $G$ qui est soit psh soit identiquement $-\infty$.

Supposons que $G \not \equiv-\infty$. Puisque $f$ est algébriquement stable, $F^{j}$ est un relèvement de $f^{j}$ pour tout $j$ et $G_{j}$ est donc un potentiel de $\rho_{+}^{-j}\left(f^{j}\right)^{*}\left(\omega^{\prime}\right)$; cette dernière suite converge donc au sens des courants vers un courant $T$ qui admet $G$ pour potentiel. Puisque $G_{j} \circ F=\rho_{+} \cdot G_{j+1}$ et $G_{j}(\lambda z, \mu w)=$ $a \log |\lambda|+b \log |\mu|+G_{j}(z, w)$, on en déduit les relations annoncées pour $G$ qui impliquent que $T$ est cohomologue à $\omega^{\prime}$ et $f^{*}(T)=\rho_{+} \cdot T$.

Montrons qu'on n'a pas $G \equiv-\infty$. Considérons

$$
\sigma_{p}=\frac{1}{p} \sum_{j=1}^{p} \frac{1}{\rho_{+}^{j}}\left[a\left(f^{j}\right)^{*}\left(\omega_{1}\right)+b\left(f^{j}\right)^{*}\left(\omega_{2}\right)\right] .
$$

Alors $\left(\sigma_{p}\right)$ est une suite bornée de courants positifs fermés de bidegré $(1,1)$ ; on peut en extraire une sous-suite convergeant vers $\sigma$. Il résulte de la définition des $\sigma_{p}$ et de la continuité de l'opération $f^{*}$ ( $f$ est dominante) que $f^{*}(\sigma)=\rho_{+} \cdot \sigma$. Soit $u$ un potentiel de $\sigma$ dans $\mathbb{C}^{n+1} \times \mathbb{C}^{m+1} ;$ l'égalité précédente implique $\rho_{+}^{-1}(u \circ F)=u+c^{\prime}$, où $c^{\prime}$ est une constante. Soit $v:=u+c^{\prime}\left(\rho_{+}-1\right)^{-1} \in P S H\left(\mathbb{C}^{n+1} \times \mathbb{C}^{m+1}\right)$, alors $v \circ F=\rho_{+} \cdot v$ et on va 
montrer que $v$ minore $G$. Comme $\sigma$ est cohomologue à $\omega^{\prime}=a \omega_{1}+b \omega_{2}$, on a $v(z, w) \leq a \log |z|+b \log |w|+C$; on en déduit

$$
v(z, w)=\frac{1}{\rho_{+}^{j}} v \circ F^{j}(z, w) \leq G_{j}(z, w)+\frac{C}{\rho_{+}^{j}},
$$

d'où $v \leq G$ et $G \not \equiv-\infty$.

Remarque 2.3. Dans le cas d'un espace multiprojectif ayant $s \geq 3$ facteurs, le théorème de Perron-Frobenius assure que toute matrice de degrés $A \in$ $\mathcal{M}_{s}(\mathbb{N})$ admet une valeur propre $\rho_{+} \geq 1$ qui majore le module de toutes les autres et un vecteur propre à coordonnées non-négatives associé à $\rho_{+}$. $L$ 'existence du courant de Green se montre alors de la même manière que dans le cas de deux facteurs, lorsque $\rho_{+}>1$.

Proposition 2.4. Soit $\mathcal{K}_{f}$ l'ensemble des courants $S$ positifs fermés de bidegré $(1,1)$ sur $\mathbb{P}^{n} \times \mathbb{P}^{m}$ tq $f^{*}(S)=\rho_{+} \cdot S$ et $\|S\|=1$. Alors le courant de Green $T$ est extrémal dans le convexe compact $\mathcal{K}_{f}$.

Démonstration. Soit $S \in \mathcal{K}_{f}$ qui est cohomologue à $x \omega_{1}+y \omega_{2} ;$ l'équation $f^{*}(S)=\rho_{+} \cdot S$ implique que $(x, y)$ est une vecteur propre associé à $\rho_{+}$et la normalisation $\|S\|=1$ entraîne $(x, y)=(a, b)$, i.e. $S$ est cohomologue à $T$.

Supposons que $T=\left(T_{1}+T_{2}\right) / 2$ avec $T_{i} \in \mathcal{K}_{f}$. On peut choisir des potentiels $G_{i}$ des $T_{i}$ tels que $G_{i} \circ F=\rho_{+} \cdot G_{i}$. La démonstration du théorème précédent montre que l'on a nécessairement $G_{i} \leq G$. Or $G$ et $G^{\prime}=\left(G_{1}+G_{2}\right) / 2$ sont des potentiels de $T$ invariants par rotation, ils diffèrent d'une constante qui doit être nulle puisque $G \circ F=\rho_{+} \cdot G, G^{\prime} \circ F=\rho_{+} \cdot G^{\prime}$ et $\rho_{+}>1$. Cela entraîne $G \equiv G_{1} \equiv G_{2}$.

Exemples 2.5. Soit $f$ l'application monomiale de $\mathbb{P}^{1} \times \mathbb{P}^{1}$ définie par

$$
\begin{aligned}
f: \mathbb{P}^{1} \times \mathbb{P}^{1} & \rightarrow \mathbb{P}^{1} \times \mathbb{P}^{1} \\
([z],[w]) & \mapsto\left(\left[z_{0}^{\alpha} w_{0}^{\beta}: z_{1}^{\alpha} w_{1}^{\beta}\right],\left[z_{0}^{\gamma} w_{0}^{\delta}: z_{1}^{\gamma} w_{1}^{\delta}\right]\right) .
\end{aligned}
$$

$C$ 'est une application algébriquement stable qui est dominante si $\operatorname{det} A \neq 0$. On peut calculer explicitement les itérés $f^{j}$ de $f$ (voir section 3 plus loin) On obtient, lorsque $\beta \gamma \neq 0$,

$$
G=\log \max \left(\left|z_{0}\right|^{a}\left|w_{0}\right|^{b} ;\left|z_{1}\right|^{a}\left|w_{1}\right|^{b}\right) \text { avec } a=\gamma / c \text { et } b=\left(\rho_{+}-\alpha\right) / c,
$$

où $c=\gamma+\left(\rho_{+}-\alpha\right)$ est telle que $\int\left(a \omega_{1}+b \omega_{2}\right) \wedge \omega^{n-m+1}=1$. Le convexe $\mathcal{K}_{f}$ sera décrit au paragraphe 3.2.

Théorème 2.6. Soit $A \in \mathcal{M}_{2}(\mathbb{N})$ avec $\beta \gamma \neq 0$ et $\rho_{+}>1$. Soit $f \in \mathcal{M}_{A}$ une application algébriquement stable. Alors

i) Le courant $T$ est à support dans l'ensemble de Julia qui est donc non vide.

ii) Pour tout compact $F$ de l'ensemble de normalité $\mathcal{N}$, on a

$$
\forall k \geq 1, \quad\left|G_{k+j}-G_{j}\right| \leq \frac{C_{F}}{\rho_{+}^{j}} \text { dans } F,
$$

et $\mathcal{N} \cap(X \backslash$ Supp $T)$ est contenu dans l'ensemble de Fatou. En particulier la fonction de Green est continue dans $\pi^{-1}(\mathcal{N})$. 
Démonstration. Il s'agit d'une adaptation immédiate de la preuve du théorème I.6.5 de [Si 99].

Corollaire 2.7. Si $f$ est normale alors le support de $T$ coïncide avec l'ensemble de Julia $J$ de $f$ qui est donc connexe.

Démonstration. Il suffit de remarquer que le complémentaire du support de $T$ est Stein dés que $T$ est cohomologue à $a \omega_{1}+b \omega_{2}$ avec $a, b>0$. Cela implique que le support de $T$ (et donc $J$ ) est connexe.

Théorème 2.8. Soit $A \in \mathcal{M}_{2}(\mathbb{N})$ avec $\beta \gamma \neq 0$ et $\rho_{+}>1$. Soit $f \in \mathcal{M}_{A}$ une application algébriquement stable. Alors $T$ ne charge pas les hypersurfaces.

Démonstration. Supposons que $T$ donne de la masse à une hypersurface $V$ de $X$. Le théorème de Siu [S 74] permet de décomposer $T=T_{1}+T_{2}$ où $T_{1}$ ne charge pas les hypersurfaces et $T_{2}=\sum_{k} c_{k}\left[V_{k}\right]$, les $V_{k}$ étant des hypersurfaces et les $c_{k}$ des constantes non-négatives non toutes nulles. Le courant $\rho_{+}^{-1} f^{*}\left(T_{2}\right)$ est supporté par une réunion d'hypersurfaces de $X$ tandis que $\rho_{+}^{-1} f^{*}\left(T_{1}\right)$ se décompose en $\rho_{+}^{-1} f^{*}\left(T_{1}\right)=T_{1}^{\prime}+T_{2}^{\prime}$ où $T_{1}^{\prime}$ ne charge aucune hypersurface et $T_{2}^{\prime}=\sum_{k} c_{k}^{\prime}\left[V_{k}^{\prime}\right]$. L'invariance de $T$ entraîne $T_{1}=T_{1}^{\prime}$ et

$$
T_{2}=T_{2}^{\prime}+\frac{1}{\rho_{+}} f^{*}\left(T_{2}\right) \geq \frac{1}{\rho_{+}} f^{*}\left(T_{2}\right)
$$

On peut itérer cette inégalité et prendre une moyenne de Césaro pour obtenir $T_{2} \geq \sigma_{p}:=p^{-1} \sum_{j=1}^{p} \rho_{+}^{-j}\left(f^{j}\right)^{*}\left(T_{2}\right)$. Cette suite de courants est de masse uniformément bornée supérieurement et inférieurement (cf lemme 2.1.iv), si $\sigma$ est une valeur d'adhérence, elle va vérifier $f^{*} \sigma=\rho_{+} \sigma \leq T_{2} \leq T$. L'extrémalité du courant $T$ (proposition 2.3) implique $\sigma=c \cdot T$ où $c$ est une constante strictement positive, mais alors $T \leq c^{-1} T_{2}$ et donc $T_{1} \equiv 0$.

Ainsi $T=T_{2}$ et on obtient la contradiction cherchée en reprenant pas à pas la fin de la démonstration du théorème I.8.1 de [Si 98].

\section{Applications monomiales}

Nous étudions dans cette section la dynamique des applications monomiales de $\mathbb{P}^{1} \times \mathbb{P}^{1}$ définies dans $\mathbb{C}^{2}$ par

$$
f(z, w)=\left(z^{\alpha} w^{\beta}, z^{\gamma} w^{\delta}\right)
$$

Une application de ce type est dominante ssi $\operatorname{det} A \neq 0$, elle est algébriquement stable dans $\mathbb{P}^{1} \times \mathbb{P}^{1}$. On supposera de plus que $f$ n'est ni holomorphe, ni un produit croisé i.e. $\beta \gamma \neq 0$ et $\alpha+\delta \neq 0$. La dynamique de ces applications est simple à décrire. Lorsqu'on les restreint au tore invariant $\mathbb{T}=\{|z|=|w|=1\}$, on obtient des endomorphismes linéaires dont la dynamique a été abondamment étudiée (cf e.g. [K-H 95]).

La fonction de Green définie par le théorème 2.2 se calcule explicitement

$$
G=\log \max \left(\left|z_{0}\right|^{a}\left|w_{0}\right|^{b} ;\left|z_{1}\right|^{a}\left|w_{1}\right|^{b}\right) .
$$

Notons $T=\mathcal{L}(G)$ le courant de Green de $f$. Dans ce cas Supp $T$ coïncide avec l'ensemble de Julia $J_{f}$. 
Les fonctions $G_{0}=\log \left(\left|z_{0}\right|^{a}\left|w_{0}\right|^{b}\right)$ et $G_{1}=\log \left(\left|z_{1}\right|^{a}\left|w_{1}\right|^{b}\right)$ vérifient également l'équation fonctionnelle $G_{i} \circ F=\rho_{+} \cdot G_{i}$. On note $T_{i}=\mathcal{L}\left(G_{i}\right)$ les courants de $\mathcal{T}\left(\mathbb{P}^{1} \times \mathbb{P}^{1}\right)$ correspondants.

Théorème 3.1. Les courants $T, T_{0}, T_{1}$ sont les points extrémaux du convexe compact

$$
\mathcal{K}_{f}:=\left\{S \in \mathcal{T}\left(\mathbb{P}^{1} \times \mathbb{P}^{1}\right) / f^{*} S=\rho_{+} \cdot S \text { et }\|S\|=1\right\} .
$$

Autrement dit, tout courant positif fermé $S$ de bidegré $(1,1)$ sur $\mathbb{P}^{1} \times \mathbb{P}^{1}$ qui vérifie $f^{*} S=\rho_{+} \cdot S$ est combinaison linéaire à coefficients non-négatifs de ces trois courants.

Démonstration. Soit $S \in \mathcal{K}_{f}$ et $\varphi$ un potentiel associé dans $\mathbb{C}^{2} \times \mathbb{C}^{2}$ t.q. $\varphi \circ F=\rho_{+} \cdot \varphi$. On ne perd rien à supposer $S$ extrémal. Supposons que $\nu(S,([1: 0],[1: 0]))=t>0$, où $\nu(S, p)$ désigne le nombre de Lelong de $S$ au point $p$. On a toujours l'inégalité $\nu\left(f^{*} S, p\right) \geq \nu(S, f(p))$. Si $p \in\left\{z_{1} w_{1}=0\right\}$, $f(p)=([1: 0],[1: 0])$, et donc $\nu(S, p) \geq \rho_{+}^{-1} \nu(S,([1: 0],[1: 0]))>0$. Donc $S$ charge $\left\{z_{1}=0\right\}$ et $\left\{w_{1}=0\right\}$. L'extrémalité et l'invariance de $S$ assurent alors que $S=T_{1}$. On obtiendra de même $S=T_{0}$ si $\nu(S,([0: 1],[0: 1]))>0$.

On suppose à présent que ces deux nombres de Lelong sont nuls. On note $\varphi_{0}$ la restriction de $\varphi$ dans la carte $\left\{z_{0}=w_{0}=1\right\} \simeq \mathbb{C}^{2}$, i.e. $\varphi_{0}(z, w)=$ $\varphi(1, z, 1, w)$. Soit $\Delta^{2}=\left\{(z, w) \in \mathbb{C}^{2} /|z|,|w|<1\right\}$, clairement $\Delta^{2} \subset \Omega_{0}$. On va prouver, par des arguments d'estimées volumiques, que $\varphi_{0}$ est nulle dans $\Delta^{2}$. L'équation fonctionnelle $\varphi_{0} \circ f=\rho_{+} \cdot \varphi_{0}$ implique alors que $\varphi_{0} \equiv 0$ dans le bassin d'attraction $\Omega_{0}$ de l'origine. En considérant $\varphi_{1}(z, w)=\varphi(z, 1, w, 1)$ la restriction de $\varphi$ dans la carte $\left\{z_{1}=w_{1}=1\right\}$, un raisonnement similaire donne $\varphi_{1} \equiv 0$ dans $\Omega_{\infty}$ puisque $\nu(S,([0,1] ;[0,1]))=0$. On conclut grâce à la bihomogénéité de $\varphi$ que $\varphi \equiv G$, d'où $S=T$.

Rappelons que $\varphi \leq G$ dans $\mathbb{C}^{2} \times \mathbb{C}^{2}$ puisque $\varphi$ vérifie l'équation fonctionnelle $\varphi \circ f=\rho_{+} \cdot \varphi$ et $\varphi$ a la même bihomogénéité que $G$ (cf théorème 2.2). On a donc $\varphi_{0}(z, w) \leq 0=G(1, z, 1, w)$ dans $\Delta^{2} \subset \Omega_{0}$. Notons pour tout $t>0, E_{t}=\left\{(z, w) \in \Delta^{2}, \varphi(z, w)<-t\right\}$. On veut donc montrer que $\operatorname{Vol}\left(E_{t}\right)=0$ pour tout $t>0$. Pour cela nous utilisons l'estimation classique de théorie du pluripotentiel (voir e.g. [Ki 00]):

$$
\forall \varepsilon>0, \exists C_{\varepsilon}>0 \text { t.q. } \forall t>0, \quad \operatorname{Vol}\left(E_{t}\right) \leq C_{\varepsilon} \exp (-t / \varepsilon),
$$

ainsi que le lemme suivant.

Lemme 3.2. Soit $E \subset \Delta^{2}$ un borélien.

- Si $\alpha+\beta \neq \gamma+\delta$, il existe des constantes $C_{1}, C_{2}>0$, telles que pour tout $n \in \mathbb{N}$,

$$
\operatorname{Vol}\left(f^{n}(E)\right) \geq\left(C_{1} \operatorname{Vol}(E)\right)^{C_{2} \rho_{+}^{n}} .
$$

- Si $\alpha+\beta \neq \gamma+\delta$, il existe des constantes $C_{1}, C_{2}>0$, telles que pour tout $n \in \mathbb{N}$,

$$
\operatorname{Vol}\left(f^{n}(E)\right) \geq\left(C_{1} T\right)^{C_{2} \rho_{+}^{n}},
$$

avec $1>T>0$ determiné par la relation $-T \log T=\operatorname{Vol}(E)$. 
Achevons la démonstration du théorème munis de ces estimations. L'invariance de $\varphi$ assure que pour tout $n \in \mathbb{N}$, on a $f^{n}\left(E_{t}\right) \subset E_{t \rho_{+}^{n}}$. On en déduit, pour $t>0$ fixé:

$$
\begin{aligned}
C_{\varepsilon} \exp \left(-t \rho_{+}^{n} / \varepsilon\right) & \geq \operatorname{Vol}\left(E_{t \rho_{+}^{n}}\right) \\
& \geq \operatorname{Vol}\left(f^{n}\left(E_{t}\right)\right) \\
& \geq\left(C_{1} x\left(E_{t}\right)\right)^{C_{2} \rho_{+}^{n}},
\end{aligned}
$$

avec $x\left(E_{t}\right)=\operatorname{Vol}\left(E_{t}\right)$ dans le cas $\alpha+\beta \neq \gamma+\delta$, et $x\left(E_{t}\right)=T$ défini par la relation $-T \log T=\operatorname{Vol}\left(E_{t}\right)$ sinon. Dans les deux cas, on a $x\left(E_{t}\right)=0$ ssi $\operatorname{Vol}\left(E_{t}\right)=0$. Si l'on choisit $\varepsilon \ll 1$ assez petit, la comparaison des croissances, lorsque $n$ tend vers l'infini, implique $\operatorname{Vol}\left(E_{t}\right)=0$, ce qui termine la démonstration.

Démonstration du lemme 3.2. Dans le cours de la preuve nous aurons besoin du lemme suivant, dont nous laissons la preuve au lecteur.

Lemme 3.3. Fixons $a, b>0, R>0$ et $t>0$. Notons $\tau=a / b$, et $V_{R}(a, b, t)=\operatorname{Vol}\left(\left\{|z|<R,|w|<R,|z|^{a}|w|^{b}<t\right\}\right)$. On a alors

- Si $\tau \neq 1$,

$$
V_{R}(a, b, t)=\frac{\pi^{2}}{1-\tau^{-1}} R^{2\left(1-\tau^{-1}\right)} t^{2 / a}+\frac{\pi^{2}}{1-\tau} R^{2(1-\tau)} t^{2 / b}
$$

- si $\tau=1(a=b)$,

$$
V_{R}(a, a, t)=\pi^{2} t^{2 / a}\left(1+\log \left(R^{4} / t^{2 / a}\right)\right) .
$$

Soit $E \subset \Delta^{2}$ un borélien et $n \in \mathbb{N}$. On note $\lambda$ la mesure de Lebesgue de $\mathbb{C}^{2}, J f$ le déterminant jacobien de $f$ et $f^{n}(z, w)=\left(z^{\alpha_{n}} w^{\beta_{n}}, z^{\gamma_{n}} w^{d_{n}}\right)$. On a

$$
\begin{aligned}
\operatorname{Vol}\left(f^{n}(E)\right) & =\int_{f^{n}(E)} d \lambda=d_{t}^{-n} \int_{E}\left|J f^{n}\right|^{2} d \lambda \\
& =d_{t}^{-n} \int_{0}^{\infty} \lambda\left(E \cap\left\{\left|J f^{n}\right|^{2}>t\right\}\right) d t \\
& \geq d_{t}^{-n} \int_{0}^{T_{0}} \operatorname{Vol}(E)-\lambda\left(E \cap\left\{\left|J f^{n}\right|^{2} \leq t\right\}\right) d t \\
& \geq d_{t}^{-n} \int_{0}^{T_{0}} \operatorname{Vol}(E)-\lambda\left(\left\{\left|J f^{n}\right|^{2} \leq t\right\}\right) d t,
\end{aligned}
$$

où $T_{0}>0$ sera choisi ultérieurement de sorte que $\operatorname{Vol}(E) \simeq \lambda\left\{\left|J f^{n}\right|^{2} \leq T_{0}\right\}$. Il nous faut à présent majorer $\lambda\left(\left\{\left|J f^{n}\right|^{2} \leq t\right\}\right)$. Un calcul immédiat donne

$$
J f^{n}(z, w)=(\operatorname{det} A)^{n} \times z^{\alpha_{n}+\gamma_{n}-1} w^{\beta_{n}+\delta_{n}-1} .
$$

Donc

$$
\lambda\left\{\left|J f^{n}\right|^{2} \leq t\right\}=V_{1}\left(\alpha_{n}+\gamma_{n}-1, \beta_{n}+\delta_{n}-1, \sqrt{t} /|\operatorname{det} A|^{n}\right) .
$$

On peut donc estimer $\lambda\left\{\left|J f^{n}\right|^{2} \leq t\right\}$ grâce au lemme 3.4. Comme on travaille dans $\Delta^{2}$, il suffit d'estimer $\lambda\left\{\left|J f^{n}\right|^{2} \leq t\right\}$ pour $t /|\operatorname{det} A|^{2 n}<1$.

Fixons $D_{2}>0$ telle que $\max \left\{\alpha_{n}+\gamma_{n}-1, \beta_{n}+\delta_{n}-1\right\} \leq D_{2} \rho_{+}^{n}$ et soit $\tau_{n}=\left(\alpha_{n}+\gamma_{n}-1\right)\left(\beta_{n}+\delta_{n}-1\right)^{-1}$. Deux cas se présentent. 
Lorsque $\alpha+\gamma \neq \beta+\delta$, la suite $\tau_{n}$ tend vers une valeur $\tau_{\infty} \neq 1$, et ne prend jamais la valeur 1. Par 3.4, il existe donc $D_{1}>1$ telle que

$$
\lambda\left\{\left|J f^{n}\right|^{2} \leq t\right\} \leq D_{1}\left(\frac{t}{|\operatorname{det} A|^{2 n}}\right)^{1 / D_{2} \rho_{+}^{n}} .
$$

On choisit maintenant $T_{0}>0$ tel que

$$
D_{1}|\operatorname{det} A|^{-2 n / D_{2} \rho_{+}^{n}} T_{0}^{1 / D_{2} \rho_{+}^{n}}=\operatorname{Vol}(E) .
$$

On obtient

$$
\begin{aligned}
\operatorname{Vol}\left(f^{n}(E)\right) & \geq d_{t}^{-n}\left(T_{0} \operatorname{Vol}(E)-D_{1}|\operatorname{det} A|^{-2 n / D_{2} \rho_{+}^{n}} \int_{0}^{T_{0}} t^{1 / D_{2} \rho_{+}^{n}} d t\right) \\
& =d_{t}^{-n} T_{0}\left(\operatorname{Vol}(E)-\frac{D_{1}|\operatorname{det} A|^{-2 n / D_{2} \rho_{+}^{n} T_{0}^{1 / D_{2} \rho_{+}^{n}}}}{1+1 / D_{2} \rho_{+}^{n}}\right) \\
& =d_{t}^{-n} \operatorname{Vol}(E) T_{0} \times\left(D_{2} \rho_{+}^{n}+1\right)^{-1} \\
& =\frac{|\operatorname{det} A|^{n} \operatorname{Vol}(E)}{1+D_{2} \rho_{+}^{n}}\left(\frac{\operatorname{Vol}(E)}{D_{1}}\right)^{D_{2} \rho_{+}^{n}} \\
& \geq\left(\frac{\operatorname{Vol}(E)}{D_{1}^{\prime}}\right)^{D_{2}^{\prime} \rho_{+}^{n}}
\end{aligned}
$$

ce qui prouve l'estimée 2 .

Lorsque $\alpha+\gamma=\beta+\delta$, on a pour tout $n \in \mathbb{N}, \tau_{n}=1$. On notera dans la suite $g(u):=-\pi^{2} u \log (u / e)$. La fonction $g$ est positive sur $[0,1]$ et strictement croissante. Par 3.4, on a

$$
\lambda\left\{\left|J f^{n}\right|^{2} \leq t\right\} \leq g\left(\left(t /|\operatorname{det} A|^{2 n}\right)^{1 / D_{2} \rho_{+}^{n}}\right) .
$$

On fixe alors $T_{0}>0$ tel que $D_{1} g\left(\left(T_{0} /|\operatorname{det} A|^{2 n}\right)^{1 / D_{2} \rho_{+}^{n}}\right)=\operatorname{Vol}(E)$. On obtient de manière analogue au cas précédent

$$
\begin{aligned}
\operatorname{Vol}\left(f^{n}(E)\right) & \geq d_{t}^{-n}\left(T_{0} \operatorname{Vol}(E)-\int_{0}^{T_{0}} D_{1} g\left(\left(t /|\operatorname{det} A|^{2 n}\right)^{1 / D_{2} \rho_{+}^{n}}\right) d t\right) \\
& \geq C d_{t}^{-n} \operatorname{Vol}(E) T_{0} \times \frac{1}{\rho_{+}^{n}+1} .
\end{aligned}
$$

Si $T_{1}>0$ est défini par l'équation $-\pi^{2} D_{1} T_{1} \log \left(T_{1} / e\right)=\operatorname{Vol}(E)$, on a

$$
T_{0}=T_{1}^{D_{2} \rho_{+}^{n}}|\operatorname{det} A|^{2 n} .
$$

Si $T>0$ est solution de $-T \log T=\operatorname{Vol}(E)$, on a donc pour un choix adéquat de $C_{1}>0$,

$$
\operatorname{Vol}\left(f^{n}(E)\right) \geq\left(C_{1} T\right)^{D_{2}^{\prime} \rho_{+}^{n}},
$$

ce qui conclut la preuve de 3.2 . 


\section{Mesure limite dans $\mathbb{P}^{1} \times \mathbb{P}^{1}$}

\subsection{Degrés dynamiques.}

Soit $g: X \rightarrow X$ une application méromorphe dominante sur une variété projective $X$. L'application $g$ induit une application linéaire sur le sousespace de $H^{l, l}(X)$ engendré par les cycles analytiques de dimension $l$ (cf [F 91]). On note $r_{l}(g)$ le rayon spectral de cette application linéaire. Si $f: X \rightarrow X$ est une autre application méromorphe dominante, on vérifie (cf lemma 3 p.364 [F 91], voir également lemma 4.6 p.916 [R-S 97]) que

$$
r_{l}(g \circ f) \leq r_{l}(f) \cdot r_{l}(g) .
$$

En particulier la suite $\left\{r_{l}\left(g^{j}\right)\right\}_{j \in \mathbb{N}}$ est sous-multiplicative, ce qui assure la convergence de la suite $\left(r_{l}\left(g^{j}\right)\right)^{1 / j}$.

Définition 4.1. Soit $g: X \rightarrow X$ une application méromorphe dominante sur une variété projective $X$ et $1 \leq l \leq k=\operatorname{dim}_{\mathbb{C}} X$.

On définit le lième degré dynamique de $g$ par:

$$
\lambda_{l}(g):=\lim _{j \rightarrow+\infty}\left(r_{l}\left(g^{j}\right)\right)^{1 / j} .
$$

Lorsque $l=k=\operatorname{dim}_{\mathbb{C}} X, \lambda_{k}(g)=r_{k}(g)$ est le degré topologique de $f$ (i.e. le nombre de préimages par $f$ d'un point générique) que l'on notera $d_{t}(f)$.

Exemples 4.2. Soit $g: \mathbb{P}^{k} \rightarrow \mathbb{P}^{k}$ une application méromorphe dominante et $\omega_{F S}$ la forme de Kähler de Fubini-Study sur $\mathbb{P}^{k}$. Alors $g^{*}\left(\omega_{F S}^{l}\right)$ est une forme lisse de bidegré $(l, l)$ bien définie dans $\mathbb{P}^{k} \backslash I_{g}$ dont la masse est finie. On note encore $g^{*}\left(\omega_{F S}^{l}\right)$ son extension triviale à travers $I_{g}$, c'est un courant positif fermé de bidegré (l,l) sur $\mathbb{P}^{k}$ (cf [Sk 82]). La classe de cohomologie du courant $g^{*}\left(\omega_{F S}^{l}\right)$ est précisément $r_{l}(g)\left[\omega_{F S}^{l}\right]$. Russakovskii et Shiffman ont établi certaines inégalités entre les différents degrés dynamiques.

Exemples 4.3. Soit $A \in \mathcal{M}_{2}(\mathbb{N})$ et $f \in \mathcal{M}_{A}$ une application méromorphe dominante de $\mathbb{P}^{1} \times \mathbb{P}^{1}$. L'application linéaire induite sur l'espace $H^{1,1}\left(\mathbb{P}^{1} \times\right.$ $\left.\mathbb{P}^{1}, \mathbb{R}\right) \simeq \mathbb{R}^{2}$ est donnée -dans la base $\left(\omega_{1}, \omega_{2}\right)$ - par la matrice $A$. On a donc $r_{1}(f)=\rho_{+}$, et $\lambda_{1}(f)=\rho_{+}$si et seulement si $f$ est algébriquement stable (cf proposition 1.6).

Lemme 4.4. Les degrés dynamiques $\lambda_{l}$ sont invariants par conjugaison birationnelle.

Démonstration. Soit $g: X \rightarrow X$ une application méromorphe dominante, $\Phi: X \rightarrow X$ une application birationnelle et $f:=\Phi \circ g \circ \Phi^{-1}$. On a $f^{j}=\Phi \circ g^{j} \circ \Phi^{-1}$ donc $r_{l}\left(f^{j}\right) \leq r_{l}(\Phi) r_{l}\left(\Phi^{-1}\right) r_{l}\left(g^{j}\right)$ (d'après (4)) d'où $\lambda_{l}(f) \leq \lambda_{l}(g)$. On obtient l'inégalité inverse en intervertissant les rôles de $f$ et $g$ et en changeant $\Phi$ en $\Phi^{-1}$.

Définition 4.5. Soit $f: X \rightarrow X$ une application méromorphe dominante, et $\mu$ une mesure borélienne de masse finie sur $X$. Soit $V \supset I_{f}$ le sousensemble des points de $X$ en lesquels $f$ n'est pas finie. On définit $f^{*} \mu$ comme l'extension triviale à travers $V$ de $\left(\left.f\right|_{X \backslash V}\right)^{*}\left(\left.\mu\right|_{X \backslash f(V)}\right)$. 
Soit $g: \mathbb{P}^{2} \rightarrow \mathbb{P}^{2}$ une application méromorphe dominante qui vérifie $\lambda_{1}(g)<d_{t}(g)$. Russakovski et Shiffman ont montré (theorem 1.1 p.899 [R-S 97]) l'existence d'une mesure de probabilité $\mu_{g}$ sur $\mathbb{P}^{2}$ et d'un ensemble pluripolaire $\mathcal{E}_{g}$ de $\mathbb{P}^{2}$ tels que

$$
\forall a \in \mathbb{P}^{2} \backslash \mathcal{E}_{g}, \frac{1}{\left(d_{t}(g)\right)^{j}}\left(g^{j}\right)^{*}\left(\varepsilon_{a}\right) \rightarrow \mu_{g},
$$

où $\varepsilon_{a}$ désigne la masse de Dirac au point $a$. On peut transporter ce résultat dans $\mathbb{P}^{1} \times \mathbb{P}^{1}$ via des conjugaisons birationnelles:

Théorème 4.6 (Russakovskii-Shiffman). Soit $A \in \mathcal{M}_{2}(\mathbb{N})$ et $f \in \mathcal{M}_{A}$ une application méromorphe dominante de $\mathbb{P}^{1} \times \mathbb{P}^{1}$.

Si $\lambda_{1}(f)<d_{t}(f)$ alors il existe une mesure de probabilité $\mu_{f}$ sur $\mathbb{P}^{1} \times \mathbb{P}^{1}$ et un ensemble pluripolaire $\mathcal{E}_{f}$ de $\mathbb{P}^{1} \times \mathbb{P}^{1}$ tels que

$$
\frac{1}{\left(d_{t}(f)\right)^{j}}\left(f^{j}\right)^{*}\left(\varepsilon_{a}\right) \rightarrow \mu_{f},
$$

pour tout $a \in \mathbb{P}^{1} \times \mathbb{P}^{1} \backslash \mathcal{E}_{f}$.

Proposition 4.7. Soit $A \in \mathcal{M}_{2}(\mathbb{N})$ et $f \in \mathcal{M}_{A}$ une application méromorphe dominante de $\mathbb{P}^{1} \times \mathbb{P}^{1}$. On note $d_{t}(f)$ son degré topologique, i.e. le nombre de préimages par $f$ d'un point générique. Alors

$$
\begin{cases}d_{t}(f)=\alpha \delta+\beta \gamma & \text { si } I_{P} \cap I_{Q}=\emptyset \\ d_{t}(f)<\alpha \delta+\beta \gamma & \text { si } I_{P} \cap I_{Q} \neq \emptyset .\end{cases}
$$

En particulier $d_{t}(f)<\rho_{+}^{2}$ à moins que $\alpha=\delta=0$ ou $\alpha=\delta \geq 1$ et $\beta \gamma=0$.

Démonstration. Soit $p=(x, y)$ un point générique de $\mathbb{P}^{1} \times \mathbb{P}^{1}$ et $\varepsilon_{p}=$ $\omega_{1}^{x} \wedge \omega_{2}^{y}$ la masse de Dirac au point $p$; le degré topologique de $f$ est égal à la masse de la mesure $f^{*}\left(\varepsilon_{p}\right)$ dans $\mathbb{P}^{1} \times \mathbb{P}^{1}$. Cette mesure est égale à la mesure $f^{*}\left(\omega_{1}^{x}\right) \wedge f^{*}\left(\omega_{2}^{y}\right)$ dans $\left(\mathbb{P}^{1} \times \mathbb{P}^{1}\right) \backslash I_{f}$, mais il se peut que cette dernière charge $I_{f}$. En fait elle charge précisément les points d'indétermination du second type $I_{P} \cap I_{Q}$. Comme $\omega_{1}^{x}\left(\right.$ resp. $\left.\omega_{2}^{y}\right)$ est cohomologue à $\omega_{1}\left(\right.$ resp. $\left.\omega_{2}\right)$, on a

$$
d_{t}(f) \leq \int_{\mathbb{P}^{1} \times \mathbb{P}^{1}} f^{*}\left(\omega_{1}^{x}\right) \wedge f^{*}\left(\omega_{2}^{y}\right)=\int_{\mathbb{P}^{1} \times \mathbb{P}^{1}} f^{*}\left(\omega_{1}\right) \wedge f^{*}\left(\omega_{2}\right)=\alpha \delta+\beta \gamma,
$$

avec égalité si et seulement si $I_{P} \cap I_{Q}=\emptyset$. On a donc

$\rho_{+}^{2} \geq\left((\alpha+\delta)^{2}+(\alpha-\delta)^{2}+4 \beta \gamma\right) / 4=\left(\alpha^{2}+\delta^{2}\right) / 2+\beta \gamma \geq \alpha \delta+\beta \gamma \geq d_{t}(f)$ avec inégalité stricte à moins que $\alpha=\delta=0$ (on a alors $d_{t}(f)=\rho_{+}^{2}=\beta \gamma$ ) ou $\alpha=\delta \geq 1$ et $\beta \gamma=0$ (on a alors $d_{t}(f)=\rho_{+}^{2}=\alpha^{2}$ ).

Remarque 4.8. Lorsque $f$ est algébriquement stable, on est tenté de construire une mesure dynamiquement intéressante en cherchant à définir $T_{f} \wedge$ $T_{f}$. Une telle mesure serait portée par l'ensemble $E_{f}$ lorsque $d_{t}(f)<\rho_{+}^{2}$.

Proposition 4.9. Soit $A \in \mathcal{M}_{2}(\mathbb{N})$ et $f \in \mathcal{M}_{A}$ une application méromorphe dominante algébriquement stable de $\mathbb{P}^{1} \times \mathbb{P}^{1}$ telle que $I_{P} \cap I_{Q}=\emptyset$. Alors $d_{t}(f)>\lambda_{1}(f)$ sauf dans les situations suivantes:

(1) $f$ est un produit croisé $(\beta \gamma=0)$ et $\min (\alpha, \delta)=1$;

(2) $\alpha \delta=0$ et $\beta \gamma \leq \max (\alpha, \delta)+1$; 
(3) $\alpha=\beta=\gamma=\delta=1$.

En particulier si $A \in \mathcal{M}_{2}\left(\mathbb{N}^{*}\right)$ est de déterminant non nul, l'ensemble des $f \in \mathcal{M}_{A}$ telles que $d_{t}(f) \leq \lambda_{1}(f)$ est inclus dans une réunion dénombrable d'hypersurfaces de $\mathcal{M}_{A}$.

Démonstration. Immédiat.

Exemples 4.10. Soit $f \in \mathcal{M}_{A}$ l'application monomiale définie en section 3 avec $\operatorname{det} A \neq 0$. On a alors $d_{t}(f)=|\alpha \delta-\beta \gamma|=\rho_{+} \cdot\left|\rho_{-}\right|$. Dans ce cas l'inégalité $d_{t}(f)>\lambda_{1}(f)$ équivaut à $\left|\rho_{-}\right|>1$ et la mesure limite $\mu_{f}$ est la mesure de Lebesgue sur le tore $\mathbb{T}=\{|z|=|w|=1\}$.

On a malheureusement très peu d'informations sur la mesure limite $\mu_{f}$ définie au théorème 4.4. Par exemple on ne sait pas si elle est invariante (au sens $f^{*} \mu_{f}=d_{t}(f) \cdot \mu_{f}$, cf question 2 p.908 [R-S 97]). L'exemple 4 p.907 de [R-S 97] suggère en effet que le support de $\mu_{f}$ peut contenir des points d'indétermination. Nous exhiberons de tels exemples lorsque nous analyserons le cas des produits croisés (section 6). Dans ce cas, une construction alternative de $\mu_{f}$ nous assurera qu'elle ne charge pas les ensembles pluripolaires, ce qui, grâce à la propriété d'équidistribution du théorème 4.4, impliquera que $\mu_{f}$ est mélangeante. En effet, on a le

Lemme 4.11. Soit $f: \mathbb{P}^{1} \times \mathbb{P}^{1} \rightarrow \mathbb{P}^{1} \times \mathbb{P}^{1}$ une application méromorphe dominante, et soit $\mu$ une mesure de probabilité qui ne charge pas les ensembles pluripolaires vérifiant $f^{*} \mu=d_{t} \mu$ ( $\mu$ est donc invariante).

Si $\mu$ satisfait (5) alors elle est mélangeante.

Démonstration. Etant données deux fonctions test $\chi$ et $\theta$ dans $\mathbb{P}^{1} \times \mathbb{P}^{1}$, il s'agit de démontrer que

$$
<\theta \cdot \chi \circ f^{j}, \mu>:=\int \theta \cdot \chi \circ f^{j} d \mu \longrightarrow \int \chi d \mu \cdot \int \theta d \mu .
$$

Or

$$
\begin{aligned}
<\theta \cdot \chi \circ f^{j}, \mu> & =<\theta, d_{t}^{-j}\left(f^{j}\right)^{*}(\chi \mu)> \\
& =<d_{t}^{-j}\left(f^{j}\right)_{*}(\theta), \chi \mu>,
\end{aligned}
$$

et $d_{t}^{-j}\left(f^{j}\right)_{*}(\theta)(a)=<d_{t}^{-j}\left(f^{j}\right)^{*}\left(\varepsilon_{a}\right), \theta>\rightarrow \int \theta d \mu, \forall a \in \mathbb{P}^{1} \times \mathbb{P}^{1} \backslash \mathcal{E}_{f}$. Comme $\mu$ ne charge pas $\mathcal{E}_{f}$ qui est pluripolaire, on obtient la convergence souhaitée. 


\subsection{Théorie du pluripotentiel des courants pluripositifs.}

Nous allons construire une mesure mélangeante $\mu$ pour les produits croisés polynomiaux de $\mathbb{C}^{2}$ sous la forme $\mu:=d d^{c}(v T)$ où $T$ est un courant positif fermé de bidegré $(1,1)$ et $v$ est une fonction s.c.s. et localement bornée définie seulement sur le support de $T$. La fonction $v$ sera limite décroissante de fonctions psh globales ce qui garantit $d d^{c}(v T) \geq 0$. La mesure $\mu$ définit un courant "pluripositif" (voir [Si 85]).

Nous allons établir pour ces courants quelques résultats analogues aux propriétés classiques de la théorie du pluripotentiel initiée par Bedford et Taylor [B-T 82]. Bien qu'il s'agisse d'une adaptation immédiate de la théorie telle qu'elle est présentée e.g. dans [De 93], nous en donnons des démonstrations pour la commodité du lecteur.

Dans tout ce paragraphe, $T$ est un courant positif fermé de bidegré $(1,1)$ défini sur un ouvert $\Omega$ de $\mathbb{C}^{2}$. On notera $|T|:=T \wedge d d^{c} \psi$ la mesure trace du courant $T$ pour une fonction $\psi$ lisse strictement psh donnée.

Définition 4.12. Une fonction $v$ s.c.s définie sur le support de $T$ est dite $T$-psh si la condition suivante est satisfaite. Il existe une suite $\left\{v_{j}\right\}_{j}>0$ de fonctions psh dans $\Omega$ t.q. la suite $\left.v_{j}\right|_{\text {Supp } T}$ est décroissante et $\left.v_{j}\right|_{\text {Supp } T} \rightarrow v$ simplement.

Lemme 4.13. Soit $v$ une fonction T-psh localement bornée. Alors $v \in$ $L^{1}(|T|)$ et on peut donc définir le courant $d d^{c}(v T)$. Celui-ci définit une mesure positive dans $\Omega$.

Démonstration du lemme 4.13 La fonction $v$ étant localement bornée, on a bien $v \in L^{1}(|T|)$. Le courant $T$ peut être identifié localement à une forme différentielle dont les coefficients sont des mesures. Leur masse est dominée par la mesure trace $|T|$ (voir [De 93]). On peut donc définir le produit $v T$, et donc la mesure $d d^{c}(v T)$.

Quitte à régulariser les fonctions psh $v_{j}$ on peut supposer que $v_{j} \in \mathcal{C}^{\infty}(\Omega)$. Pour tout $j \geq 0$, on a $v_{j} \in L^{1}(|T|)$. Par convergence monotone, on a $v_{j} \rightarrow v$ dans $L^{1}(|T|)$ donc $v_{j} T$ converge faiblement vers $v T$. On a donc $d d^{c}\left(v_{j} T\right) \rightarrow d d^{c}(v T)$ au sens des mesures. Comme $v_{j} \in P S H(\Omega)$ on a $d d^{c}\left(v_{j} T\right)=d d^{c} v_{j} \wedge T \geq 0$. Il s'ensuit $d d^{c}(v T) \geq 0$.

Il résulte de la définition que si $v_{1}, v_{2}$ sont deux fonctions bornées $T$-psh, alors la fonction $\max \left\{v_{1}, v_{2}\right\}$ est encore $T$-psh bornée.

On va démontrer un analogue des inégalités de Chern-Levine-Nirenberg pour les fonctions $T$-psh.

Proposition 4.14. Soit $\varphi \in P S H(\Omega)$ et $v$ une fonction $T$-psh localement bornée. Fixons $K \subset \subset \Omega$ un compact.

Pour tout compact $F \subset \operatorname{Int}(K)$, il existe une constante $C>0$ indépendante de $T$ et $v$ telle que

$$
\left\|\varphi d d^{c}(v T)\right\|_{F} \leq C\|v\|_{L^{\infty}(K \cap \operatorname{Supp} T)} \cdot \int_{K} \varphi|T| .
$$

En particulier $d d^{c} v \wedge T$ ne charge pas les ensembles pluripolaires si $T$ ne les charge pas (e.g. lorsque $T=d d^{c} u$ et u est localement bornée).

\section{Démonstration de la proposition 4.14}


Quitte à réduire $\Omega$, on peut toujours supposer $K=\Omega$. Pour des raisons techniques on est amené à travailler dans $\mathbb{C}^{3}$; on note $\widetilde{T}, \widetilde{v}, \widetilde{\varphi}$ les courants et fonctions obtenus à partir de $T, v, \varphi$ en ajoutant une coordonnée indépendante, et on pose $\widetilde{F}:=F \times \Delta(1)$ et $\widetilde{\Omega}=\Omega \times \Delta(2)$. On notera abusivement $\psi$ une fonction lisse psh d'exhaustion de $\widetilde{\Omega}$. On ne perd rien à supposer que $\widetilde{\varphi} \leq 0$ et $-1 \leq \psi \leq 0$ sur $\widetilde{\Omega}$. Pour $\alpha>0$ on notera $\widetilde{\Omega}_{\alpha}:=\{\psi<-\alpha\} \subset \subset \widetilde{\Omega}$.

Fixons $\varepsilon>0$ assez petit. Quitte à retrancher $\|v\|_{L^{\infty}(\Omega \cap S u p p T)}+\varepsilon$ à $\widetilde{v}$ on peut toujours supposer que $v<-\varepsilon \operatorname{sur} \operatorname{Supp} T$.

Fixons $\delta>0$ petit t.q. $\widetilde{F} \subset \subset \widetilde{\Omega}_{\delta}$ et posons $A:=\delta^{-1}\|v\|_{L^{\infty}(\Omega \cap \operatorname{Supp} T)}$. Définissons la fonction psh dans $\widetilde{\Omega}$ par

$$
V:=\max \{\widetilde{v}, A \psi\}
$$

Notons que

- $\operatorname{sur}(\widetilde{\Omega} \backslash \widetilde{\Omega} \eta) \cap \operatorname{Supp} \widetilde{T}$, on a $V=A \psi$ avec $\eta:=\varepsilon / A$;

- $\operatorname{sur} \widetilde{\Omega}_{\delta} \cap \operatorname{Supp} \widetilde{T}$, on a $V=\widetilde{v}$.

On fixe de plus une fonction lisse $\chi$ à support compact dans $\widetilde{\Omega}$ t.q. $\chi \equiv$ $\psi+1 \geq 0$ dans $\widetilde{\Omega}_{\eta}$.

On a alors la suite d'inégalités:

$$
\begin{aligned}
0 & \leq \int_{F}-\varphi d d^{c} v \wedge T \leq C_{1} \int_{\widetilde{\Omega}_{\delta}}-\widetilde{\varphi} d d^{c} \widetilde{v} \wedge \widetilde{T} \wedge d d^{c} \psi \\
& =C_{1} \int_{\widetilde{\Omega}_{\delta}}-\widetilde{\varphi} d d^{c} V \wedge \widetilde{T} \wedge d d^{c} \psi \leq C_{1} \int_{\widetilde{\Omega}_{\eta}}-\widetilde{\varphi} d d^{c} V \wedge \widetilde{T} \wedge d d^{c} \psi \\
& =C_{1} \int_{\widetilde{\Omega}_{\eta}}-\widetilde{\varphi} d d^{c} V \wedge \widetilde{T} \wedge d d^{c} \chi=C_{1} \int_{\widetilde{\Omega}}+C_{1} \int_{\widetilde{\Omega} \backslash \widetilde{\Omega}_{\eta}} \\
& =C_{1} \int_{\widetilde{\Omega}}-\chi d d^{c} \widetilde{\varphi} \wedge d d^{c} V \wedge \widetilde{T}+C_{1} A \int_{\widetilde{\Omega} \backslash \widetilde{\Omega}_{\eta}} \widetilde{\varphi} d d^{c} \psi \wedge \widetilde{T} \wedge d d^{c} \chi \\
& \leq 0+C_{1} \frac{\|v\|_{L^{\infty}(\operatorname{Supp} T \cap \Omega)}}{\delta} \times\|\chi\|_{\mathcal{C}^{2}(\widetilde{\Omega})}\left|\int_{\Omega} \varphi T \wedge\left(d d^{c} \psi\right)^{2}\right|
\end{aligned}
$$

ce qui conclut la preuve.

Lorsque $T=d d^{c} u$ avec $u$ localement bornée, les inégalités standard de Chern-Levine-Nirenberg assurent que $d d^{c} u$ ne charge pas les ensembles pluripolaires (cf [De 93]).

Proposition 4.15. Soit $\left(u_{j}\right)$ une suite de fonctions psh dans $\Omega$ qui con verge vers $u$ dans $L_{l o c}^{1}(\Omega)$. Soit $\left(v_{j}\right)$ une suite de fonctions continues dans $\Omega$ qui décrot vers une fonction $v$, continue dans $\Omega$. Alors

$$
v_{j} d d^{c} u_{j} \rightarrow v d d^{c} u \text { au sens des courants, }
$$

et donc $d d^{c} u_{j} \wedge d d^{c} v_{j} \rightarrow d d^{c} u \wedge d d^{c} v$.

\section{Démonstration de la proposition 4.15}

Quitte à retrancher une constante, on peut supposer que $v_{j}, v \leq 0$. Soit $\theta$ une forme test positive de bidegré $(1,1)$ dans $\Omega$, il suffit de montrer que $\int_{\Omega} v_{j} d d^{c} u_{j} \wedge \theta \rightarrow \int_{\Omega} v d d^{c} u \wedge \theta$. La suite de courants $v_{j} d d^{c} u_{j}$ est de masse 
localement uniformément bornée dans $\Omega$, soit $R=\lim v_{j_{k}} d d^{c} u_{j_{k}}$ une valeur d'adhérence. On a

$$
0 \geq \int_{\Omega} v_{j_{k}} d d^{c} u_{j_{k}} \wedge \theta \geq \int_{\Omega} v d d^{c} u_{j_{k}} \wedge \theta .
$$

Or $d d^{c} u_{j_{k}} \wedge \theta$ tend vers $d d^{c} u \wedge \theta$ au sens des mesures de Radon et $v$ est continue, donc le membre de droite de l'inégalité tend vers $\int_{\Omega} v d d^{c} u \wedge \theta$. Ainsi $R \geq v d d^{c} u$. Réciproquement, on a

$$
\int_{\Omega} v_{j_{k}} d d^{c} u_{j_{k}} \wedge \theta \leq \int_{\Omega} v_{p} d d^{c} u_{j_{k}} \wedge \theta
$$

pour tout $p \leq j_{k}$ fixé. Comme $v_{p}$ est continue, le membre de droite tend vers $\int_{\Omega} v_{p} d d^{c} u \wedge \theta$; le théorème de la convergence monotone assure que ce dernier tend vers $\int_{\Omega} v d d^{c} u \wedge \theta$ lorsque $p \rightarrow+\infty$. On en déduit $R \leq v d d^{c} u$.

\subsection{Application à la dynamique dans $\mathbb{P}^{1} \times \mathbb{P}^{1}$.}

Nous allons montrer, sous certaines hypothèses, que la mesure $\mu_{f}$ ne charge pas l'ensemble de normalité.

Théorème 4.16. Soit $A \in \mathcal{M}_{2}(\mathbb{N})$ telle que $\alpha \neq \delta$ et $\beta \gamma \neq 0$. Soit $f \in \mathcal{M}_{A}$ une application méromorphe dominante algébriquement stable de $\mathbb{P}^{1} \times \mathbb{P}^{1}$.

i) Si $\rho_{-}>1$ alors $(\operatorname{det} A)^{-j}\left(f^{j}\right)^{*}\left(\omega_{1} \wedge \omega_{2}\right)$ converge dans l'ensemble de normalité $\mathcal{N}_{f}$ vers une mesure positive $\mu$ qui vérifie $f^{*} \mu=(\operatorname{det} A) \cdot \mu$. En particulier si $\operatorname{det} A \neq d_{t}(f)$, soit $\mu$ est de masse infinie, soit $\mu \equiv 0$.

ii) Si $\rho_{-}<-1$ alors $(\operatorname{det} A)^{-j}\left(f^{j}\right)^{*}\left(\omega_{1} \wedge \omega_{2}\right)$ converge vers 0 dans $\mathcal{N}_{f}$.

On déduit alors de 4.6 et 4.9 le

Corollaire 4.17. Supposons que $\rho_{+}<\min \left\{|\operatorname{det} A|, d_{t}(f)\right\}, \alpha \neq \delta, \beta \gamma \neq 0$. Alors

$$
\left.\mu_{f}\right|_{\mathcal{N}_{f}}=0 .
$$

Démonstration. Soit $U$ un ouvert relativement compact de l'ensemble de normalité $\mathcal{N}_{f}$. Il existe $C>1$ telle que pour tout $(z, w) \in U$,

$$
\begin{aligned}
C^{-1}|z|^{\alpha}|w|^{\beta} & \leq|P(z, w)| \leq|z|^{\alpha}|w|^{\beta}, \\
C^{-1}|z|^{\gamma}|w|^{\delta} & \leq|Q(z, w)| \leq|z|^{\gamma}|w|^{\delta},
\end{aligned}
$$

où $F=(P, Q)$ désigne un relèvement de $f$ dans $\mathbb{C}^{2} \times \mathbb{C}^{2}$. Rappelons que le courant de Green $T$ est cohomologue à $a \omega_{1}+b \omega_{2}$ où $(a, b)$ est un vecteur propre positif associé à la valeur propre $\rho_{+}$de $A$ normalisé de telle sorte que $\int T \wedge\left(\omega_{1}+\omega_{2}\right)=1$. Soit $(x, y)$ un vecteur propre associé à la valeur propre $\rho_{-}$de $A$; c'est un multiple de $\left(\gamma, \rho_{-}-\alpha\right)$ (et $(a, b)$ est un multiple de $\left.\left(\gamma, \rho_{+}-\alpha\right)\right)$ donc $a y+b x$ est un multiple de $\gamma(\delta-\alpha)$ que l'on a supposé non nul, on choisit $(x, y)$ de telle sorte que $a y+b x=1$. Soit

$$
v_{j}(z, w):=\frac{1}{\left(\rho_{-}\right)^{j}}\left(x \log \left|P_{j}(z, w)\right|+y \log \left|Q_{j}(z, w)\right|\right) .
$$

Les inégalités (7) et (8) entranent

$$
\begin{aligned}
-\log C+\alpha \log \left|P_{j}\right|+\beta \log \left|Q_{j}\right| & \leq \log \left|P_{j+1}\right| \leq \alpha \log \left|P_{j}\right|+\beta \log \left|Q_{j}\right|, \\
-\log C+\gamma \log \left|P_{j}\right|+\delta \log \left|Q_{j}\right| & \leq \log \left|Q_{j+1}\right| \leq \gamma \log \left|P_{j}\right|+\delta \log \left|Q_{j}\right|,
\end{aligned}
$$


et ainsi $\forall(z, w) \in U$,

$$
\left|v_{j+1}(z, w)-v_{j}(z, w)\right| \leq \frac{\max (|x|,|y|) \log C}{\left|\rho_{-}\right|^{j}} .
$$

Puisque $\left|\rho_{-}\right|>1$ on en déduit la convergence uniforme dans $U$ de la suite $\left(v_{j}\right)$ vers une fonction $v$ continue mais non psh $(x y<0)$.

On note $S_{j}=\left(\rho_{-}\right)^{-j}\left(f^{j}\right)^{*}\left(x \omega_{1}+y \omega_{2}\right)$, ainsi $S_{j}$ converge au sens des courants vers un courant fermé de bidegré $(1,1) S$ tel que $\pi^{*} S=d d^{c} v$. Soit $T_{j}:=\rho_{+}^{-j}\left(f^{j}\right)^{*}\left(a \omega_{1}+b \omega_{2}\right)$ la suite de courants positifs fermés qui converge vers le courant de Green $T ; \mu_{j}:=T_{j} \wedge S_{j}=\left(\rho_{+} \rho_{-}\right)^{-j}(a y+b x)\left(f^{j}\right)^{*}\left(\omega_{1} \wedge \omega_{2}\right)$ est une suite de mesures qui converge dans $U$ vers $\mu=S \wedge T$ d'après la proposition 4.14. Si $\rho_{-}<-1$ on obtient $\mu_{2 j} \geq 0$ et $\mu_{2 j+1} \leq 0$ donc $S \wedge T \equiv 0$. Si $\rho_{-}>1, \mu$ est une mesure positive dans $U$ qui vérifie $f^{*} \mu=\rho_{+} \rho_{-} \mu=$ $\operatorname{det} A \cdot \mu$. Si $\mu$ est de masse finie $<\mu, 1><+\infty$, alors

$$
\begin{aligned}
\operatorname{det} A<\mu, 1> & =<f^{*} \mu, 1>=<\mu, f_{*} 1> \\
& =d_{t} \cdot<\mu, 1>,
\end{aligned}
$$

ce qui implique $\mu \equiv 0$ lorsque $d_{t} \neq \operatorname{det} A$. 


\section{Applications polynomiales De $\mathbb{C}^{2}$}

Soit $f:(z, w) \in \mathbb{C}^{2} \rightarrow(P(z, w), Q(z, w)) \in \mathbb{C}^{2}$ une application polynomiale de $\mathbb{C}^{2}$, où $P$ et $Q$ sont des polynômes tels que $\operatorname{deg}_{z} P=\alpha, \operatorname{deg}_{w} P=\beta$ et $\operatorname{deg}_{z} Q=\gamma, \operatorname{deg}_{w} Q=\delta$. On note $\mathcal{P}_{A}$ l'ensemble de ces applications où

$$
A=\left[\begin{array}{ll}
\alpha & \gamma \\
\beta & \delta
\end{array}\right]
$$

désigne comme d'habitude la matrice des degrés.

On peut considérer l'extension $\tilde{f}$ de $f$ à $\mathbb{P}^{1} \times \mathbb{P}^{1}$ et on vérifie aisément que le point $q=([0: 1],[0: 1])$ est un point d'indétermination sur lequel l'une des droites $\left\{z_{0}=0\right\}$ ou $\left\{w_{0}=0\right\}$ est contractée par $\widetilde{f}$ si $\operatorname{deg} P<\alpha+\beta$ ou $\operatorname{deg} Q<\gamma+\delta$. Réciproquement si $\operatorname{deg} P=\alpha+\beta$ et $\operatorname{deg} Q=\gamma+\delta$ alors $q$ est un point fixe de $\widetilde{f}$ qui n'est pas d'indétermination. Or les droites $\left\{z_{0}=0\right\}$ et $\left\{w_{0}=0\right\}$ ne peuvent être contractées que sur $q$, donc $\widetilde{f}$ est algébriquement stable. Notons que $\left\{f \in \mathcal{P}_{A} / \tilde{f}\right.$ non algébriquement stable \} est une hypersurface de $\mathcal{P}_{A}$.

Proposition 5.1. Soit $A \in \mathcal{M}_{2}(\mathbb{N})$ et $f \in \mathcal{P}_{A}$ qui s'étend en une application méromorphe algébriquement stable $\widetilde{f} \in \mathcal{M}_{A}$ de $\mathbb{P}^{1} \times \mathbb{P}^{1}$. Alors $q=([0: 1],[0$ : 1]) est un point fixe superattractif de $\widetilde{f}$ à part dans l'un des cas suivants

(1) $\alpha=\delta=0$ et $\beta=\gamma=1$, dans ce cas $\tilde{f}$ est un automorphisme linéaire ;

(2) $\beta=0$ et $\alpha=1$;

(3) $\gamma=0$ et $\delta=1$.

La preuve est immédiate. Remarquons que les deux derniers cas correspondent à des produits croisés semi-linéaires dont la dynamique se ramène essentiellement à de la dynamique en une variable. Notons également que tout un voisinage de $\left\{z_{0} w_{0}=0\right\} \backslash I_{\widetilde{f^{2}}}$ est envoyé sur $q$ par $\widetilde{f^{2}}$, lorsque $f$ n'est pas un produit croisé, ce qui implique $E_{\widetilde{f}}=I_{\widetilde{f^{2}}}$. Nous verrons à la section 6 que l'ensemble $E_{\widetilde{f}}$ peut être beaucoup plus gros dans le cas des produits croisés (cf proposition 6.8).

\subsection{Fonction de Green.}

Comme précédemment, on notera $\rho_{+}>1$ la plus grande valeur propre de $A$, et $(a, b)$ un vecteur propre à coefficients positifs associé, normalisé par $a+b=1$. On notera aussi pour $j \in \mathbb{N}, f^{j}=\left(P^{j}, Q^{j}\right)$. Enfin, on notera dans toute cette partie $\|(z, w)\|:=|z|+|w|$ si $(z, w) \in \mathbb{C}^{2}$. De même, pour $\zeta \in \mathbb{C},\|\zeta\|:=\|(1, \zeta)\|=1+|\zeta|$.

Théorème 5.2. Soit $f \in \mathcal{P}_{A}$ une application polynomiale dominante de $\mathbb{C}^{2}$ qui s'étend en une application méromorphe algébriquement stable $\widetilde{f}$ de $\mathbb{P}^{1} \times$ $\mathbb{P}^{1}$ qui n'est ni holomorphe ni un produit croisé. Soit $g(z, w):=G_{\widetilde{f}}(1, z, 1, w)$ la fonction de Green de $f$ dans $\mathbb{C}^{2}$ et $\Omega_{q}:=\left\{(z, w) \in \mathbb{C}^{2} / f^{j}(z, w) \rightarrow q=\right.$ $([0: 1],[0: 1])\}$ le bassin d'attraction de $q$ dans $\mathbb{C}^{2}$. Alors

$$
g(z, w)=\lim _{j \rightarrow \infty} \frac{1}{\rho_{+}^{j}}\left(a \log \left\|P^{j}(z, w)\right\|+b \log \left\|Q^{j}(z, w)\right\|\right),
$$


et

$$
\Omega_{q}=\left\{(z, w) \in \mathbb{C}^{2} / g(z, w)>0\right\} .
$$

En particulier la fonction de Green est continue dans $\mathbb{C}^{2}$.

Démonstration. Sous les hypothèses du théorème, on peut écrire $f=$ $(P, Q)$ avec $\left.P(z, w)=B_{1} z^{\alpha} w^{\beta}+O(\|(z, w)\|)^{\alpha+\beta-1}\right)$ et $Q(z, w)=B_{2} z^{\gamma} w^{\delta}+$ $\left.O(\|(z, w)\|)^{\gamma+\delta-1}\right)$, et $B_{1} B_{2} \neq 0$. On peut donc trouver $R>0$ assez grand, et $C>0$ tels que $\forall(z, w) \in U_{R}:=\left\{(z, w) \in \mathbb{C}^{2}, \min \{|z|,|w|\}>R\right\}$,

$$
\begin{aligned}
& C^{-1}\|z\|^{\alpha}\|w\|^{\beta} \leq\|P(z, w)\| \leq C\|z\|^{\alpha}\|w\|^{\beta}, \\
& C^{-1}\|z\|^{\gamma}\|w\|^{\delta} \leq\|Q(z, w)\| \leq C\|z\|^{\gamma}\|w\|^{\delta} .
\end{aligned}
$$

Définissons

$$
g^{j}(z, w)=\frac{1}{\rho_{+}^{j}}\left(a \log \left\|P^{j}\right\|+b \log \left\|Q^{j}\right\|\right)+\sum_{k \geq j+1} \rho_{+}^{-j-1} \log C .
$$

Les inégalités (9) et (10) impliquent que la suite $g^{j}$ est décroissante et $\mid g^{j+1}-$ $g^{j} \mid \leq \log C / \rho_{+}^{j+1}$. On en déduit que $g^{j}$ converge uniformément vers $g$ dans $U_{R}$; de plus

$$
|g-a \log \|z\|-b \log \|w\|| \leq \log C /\left(\rho_{+}-1\right) \text { dans } U_{R},
$$

donc $U_{R} \subset \Omega_{q}$ et l'invariance de $g$ entraîne $\Omega_{q} \subset\{g>0\}$.

On va prouver que $g(z, w)=0$ si $(z, w) \notin \Omega_{q}$. Quitte à augmenter $R>0$, on a $\forall|z| \leq R, \forall|w|>R$,

$$
|P(z, w)| \leq C R^{\alpha}|w|^{\beta} \text { et }|Q(z, w)| \leq C R^{\gamma}|w|^{\delta} .
$$

De même, $\forall|z|>R, \forall|w| \leq R$,

$$
|P(z, w)| \leq C R^{\beta}|z|^{\alpha} \text { et }|Q(z, w)| \leq C R^{\delta}|w|^{\gamma} .
$$

Nous utiliserons le lemme suivant, dont nous laissons la preuve au lecteur.

Lemme 5.3. Soit $k>\max (\alpha, \delta, \sqrt{\beta \gamma})$. Pour des choix de constantes $C_{1}$, $C_{2}>0$ assez grandes vérifiant $C_{1} / C_{2}=\sqrt{\gamma / \beta}$, les suites $a_{j}=C_{1} k^{j}$, et $b_{j}=C_{2} k^{j}$ satisfont, pour tout $j \in \mathbb{N}$,

$$
\begin{aligned}
a_{j}, b_{j} & \geq 1 \\
a_{j+1} & \geq \max \left\{\beta+\alpha a_{j}, \alpha+\beta b_{j}\right\}+1, \\
b_{j+1} & \geq \max \left\{\gamma+\delta b_{j}, \delta+\gamma a_{j}\right\}+1 .
\end{aligned}
$$

Fixons maintenant $(z, w) \notin \Omega_{q}$. Soit $M_{1}:=\sup _{\Delta^{2}(R)}\{\max (|P|,|Q|)\}$ et $\tau:=\max (\alpha, \beta, \gamma, \delta)$. On pose $M:=\max \left(M_{1}, R, C,|z|,|w|\right)$ et on note $\left(z_{j}, w_{j}\right)=f^{j}(z, w)$. On a donc $\forall j \geq 1,\left|z_{j}\right| \leq R$ ou $\left|w_{j}\right| \leq R$. Vérifions par récurrence que $\forall j \geq 1,\left|z_{j}\right| \leq M^{a_{j}}$ et $\left|w_{j}\right| \leq M^{b_{j}}$. L'assertion est claire pour $j=1$ puisque $a_{1}, b_{1} \geq 1$ et $M \geq \max (|z|,|w|)$. Supposons la vérifiée au rang $j \geq 1$.

Supposons $\max \left\{\left|z_{j}\right|,\left|w_{j}\right|\right\} \leq R$. Alors $\left|z_{j+1}\right| \leq M_{1} \leq M \leq M^{a_{j+1}}$ et similairement $\left|w_{j}\right| \leq M^{b_{j+1}}$.

Supposons $\left|z_{j}\right| \leq R$ et $\left|w_{j}\right|>R$. Alors $\left|z_{j+1}\right|=\left|P\left(z_{j}, w_{j}\right)\right| \leq C R^{\alpha}\left|w_{j}\right|^{\beta} \leq$ $C M^{\alpha+\beta b_{j}-1} \leq M^{a_{j+1}}$ et $\left|w_{j+1}\right|=\left|Q\left(z_{j}, w_{j}\right)\right| \leq C R^{\gamma}\left|w_{j}\right|^{\delta} \leq C M^{\gamma+\delta b_{j}-1} \leq$ $M^{b_{j+1}}$.

Enfin le cas $\left|z_{j}\right|>R$ et $\left|w_{j}\right| \leq R$ se traite de manière similaire. 
Il suffit à présent de remarquer que

$$
\rho_{+}=\frac{\alpha+\delta+\sqrt{(\alpha-\delta)^{2}+4 \beta \gamma}}{2}>\max (\alpha, \delta, \sqrt{\beta \gamma}),
$$

lorsque $\tilde{f}$ n'est ni holomorphe ni un produit croisé. En fixant $k<\rho_{+}$dans la majoration précédente, on obtient ainsi que $a_{j} / \rho_{+}^{j} \rightarrow 0$ et $b_{j} / \rho_{+}^{j} \rightarrow 0$ et donc $g(z, w)=0$.

Remarque 5.4. Bien que $g$ soit continue, l'application $f$ n'est pas nécessairement normale. Si par exemple $f(z, w)=\left(z^{\alpha} w^{\beta}+z^{\alpha}, z^{\gamma} w^{\delta}\right)$, on a $f(z, 0)=$ $\left(z^{\alpha}, 0\right)$. Un voisinage de $\left\{(z, w) \in \mathbb{C}^{2} /|z|>1\right.$ et $\left.w=0\right\}$ est attiré par le point d'indétermination $([0: 1],[1: 0])$. Cependant la croissance vers les points d'indétermination est négligeable.

Théorème 5.5. Soit $\left(f_{\lambda}\right)$ une famille d'applications polynomiales dominantes algébriquement stables de $\mathcal{P}_{A}$ dépendant continument d'un paramètre $\lambda \in \Lambda$ ( $\Lambda$ étant un espace métrique). Soit $\left(T_{\lambda}\right)$ la famille associée des courants de Green. Alors $\lambda \rightarrow T_{\lambda}$ est continue.

Démonstration. La continuité de la famille des courants de Green est équivalente à celle des potentiels $\left(g_{\lambda}\right)$ dans $\mathbb{C}^{2}$ pour la topologie $L_{l o c}^{1}$. Puisque les degrés $A$ sont fixés indépendemment de $\lambda$, il découle de la construction des potentiels que $\forall \lambda \in \Lambda, g_{\lambda}(z, w) \leq a \log \|z\|+b \log \|w\|$, donc la famille $g_{\lambda}$ est localement uniformément majorée.

Soit $\lambda_{0} \in \Lambda$ et $v$ une valeur d'adhérence de la famille $\left(g_{\lambda}\right)$ au point $\lambda_{0}$. Montrons tout d'abord que $v \leq g_{\lambda_{0}}$. Rappelons que $g_{\lambda}$ est la limite décroissante des fonctions $g^{j}(\cdot, \lambda)$ et $\forall j \in \mathbb{N}, \lambda \rightarrow g^{j}(\cdot, \lambda)$ est continue. Or un infimum de fonctions s.c.s. est s.c.s., donc $\lambda \rightarrow g(., \lambda)$ est s.c.s. et ainsi $v \leq g_{\lambda_{0}}$. Notons que cette inégalité n'a lieu a priori que pour presque tout $(z, w) \in \mathbb{C}^{2}$, mais $v$ et $g_{\lambda_{0}}$ sont psh donc $v(z, w) \leq g_{\lambda_{0}}(z, w), \forall(z, w) \in \mathbb{C}^{2}$.

L'inégalité inverse requière véritablement le fait que les $f_{\lambda}$ sont polynomiales dans $\mathbb{C}^{2}$. Les coefficients des polynômes définissant $f_{\lambda}$ varient continument avec $\lambda$. Pour $\varepsilon>0$ fixé, on peut trouver $R>0$ et une constante $C>0$ tels que $\forall \lambda \in B\left(\lambda_{0}, \varepsilon\right), \forall(z, w) \in U_{R}$,

$$
\begin{aligned}
& C^{-1}\|z\|^{\alpha}\|w\|^{\beta} \quad \leq\left|P_{\lambda}(z, w)\right| \leq C\|z\|^{\alpha}\|w\|^{\beta}, \\
& C^{-1}\|z\|^{\gamma}\|w\|^{\delta} \leq\left|Q_{\lambda}(z, w)\right| \leq C\|z\|^{\gamma}\|w\|^{\delta} .
\end{aligned}
$$

On en déduit que la suite $g^{j}(z, \lambda)$ converge uniformément par rapport à $(z, \lambda)$ dans $U_{R} \times B\left(\lambda_{0}, \varepsilon\right)$. Donc $v \equiv g_{\lambda_{0}}$ dans $U_{R}$. Mais $v$ et $g_{\lambda_{0}}$ satisfont la même équation fonctionnelle $v \circ f_{\lambda_{0}}=\rho_{+} \cdot v$, et $g_{\lambda_{0}} \circ f_{\lambda_{0}}=\rho_{+} \cdot g_{\lambda_{0}}$. On en déduit, puisque le bassin d'attraction $\Omega_{q}\left(f_{\lambda_{0}}\right)$ de $q$ relatif à $f_{\lambda_{0}}$ est égal à $\Omega_{q}\left(f_{\lambda_{0}}\right)=\bigcup_{j \geq 1} f_{\lambda_{0}}^{-j}\left(U_{R}\right)$, que $v \equiv g_{\lambda_{0}}$ dans ce bassin. Enfin on a $0 \leq v \leq g_{\lambda_{0}}$ et $g_{\lambda_{0}} \equiv 0$ à l'extérieur de $\Omega_{q}\left(f_{\lambda_{0}}\right)$, donc $v \equiv g_{\lambda_{0}}$ dans tout $\mathbb{C}^{2}$.

Nous utliserons dans la partie 7 l'estimation suivante.

Proposition 5.6. Soit $f \in \mathcal{P}_{A}$ une application polynomiale dominante de $\mathbb{C}^{2}$ qui s'étend en une application méromorphe algébriquement stable $\widetilde{f}$ de $\mathbb{P}^{1} \times \mathbb{P}^{1}$ qui n'est ni holomorphe ni un produit croisé. 
Alors, au voisinage de tout point $p_{0} \in I_{\tilde{f}}$, le courant invariant $T$ associé $\grave{a} \tilde{f}$ admet un potentiel $G$ psh t.q.

$$
G(p) \geq c \log \operatorname{dist}\left(p, p_{0}\right)+O(1)
$$

pour une constante $c>0$.

Démonstration. On peut supposer que $p_{0}=([1: 0],[0: 1])$ et, quitte à remplacer $f$ par $f^{2}$, que $\alpha, \beta, \gamma, \delta \geq 1$. Notons $G(x, y):=G_{\widetilde{f}}(1, x, y, 1)$ un potentiel de $T$ au voisinage de $p_{0}$. On cherche une constante $c>0$ t.q. pour tout $x, y$ assez petits

$$
G(x, y) \geq c \max \{\log |x|, \log |y|\}+O(1) .
$$

On remarque tout d'abord que

$$
\begin{aligned}
G(x, y)=G_{\widetilde{f}}(1, x, y, 1) & =G_{\widetilde{f}}(1, x, 1,1 / y)+b \log |y| \\
& =g(x, 1 / y)+b \log |y| \geq b \log |y|,
\end{aligned}
$$

car la fonction $g$ est positive (voir théorème 5.2). Soit $N \in \mathbb{N}^{*}$ que nous fixerons précisément par la suite, on obtient dans $\left\{|y| \geq|x|^{N}\right\}$ l'estimation

$$
G(x, y) \geq b \log |y| \geq b N^{-1} \max \{\log |x|, \log |y|\} .
$$

Il reste à minorer $G$ dans $\left\{|y| \leq|x|^{N}\right\}$. Comme $q=([0: 1],[0: 1])$ est superattractif, la fonction $G_{\widetilde{f}}$ est pluriharmonique dans un voisinage de $\pi^{-1}\{q\}$. On peut donc trouver $C_{1}>0$ t.q. pour $u, v$ assez petits

$$
G_{\widetilde{f}}(u, 1, v, 1) \geq C_{1} .
$$

Or le point $p_{0}$ est isolé dans $I_{\widetilde{f}}$, d'où $P(1, x, 0,1)=0$ ssi $x=0$. On peut donc trouver des constantes $C_{2}>0, M \in \mathbb{N}$ t.q. $|P(1, x, 0,1)| \geq C_{2}|x|^{M}$. De même, il existe $C_{2}^{\prime}>0$ et $M^{\prime} \in \mathbb{N}$ tels que $|Q(1, x, 0,1)| \geq C_{2}^{\prime}|x|^{M^{\prime}}$. On choisit $N>\max \left(M, M^{\prime}\right)$ et $\varepsilon \ll 1$. La formule de Taylor donne pour tout point de $\left\{|y| \leq|x|^{N},|x|+|y| \leq \varepsilon\right\}$

$$
|P(1, x, y, 1)| \geq C_{2}|x|^{M}-C_{3}|y| \geq C_{4}|x|^{M},
$$

pour des constantes $C_{3}, C_{4}>0$. De même $|Q(1, x, y, 1)| \geq C_{4}|x|^{M^{\prime}}$. Donc

$$
\begin{aligned}
\sup _{|y| \leq|x|^{N},|x|+|y| \leq \varepsilon}\left\{\left|\frac{y^{\beta}}{P(1, x, y, 1)}\right|, \mid\right. & \left.\frac{y^{\delta}}{Q(1, x, y, 1)} \mid\right\} \\
& \leq C_{4}^{-1}|x|^{\min \left(\beta N-M, \delta N-M^{\prime}\right)} \ll 1 .
\end{aligned}
$$

On utilise alors l'équation fonctionnelle $G_{\widetilde{f}} \circ F=\rho_{+} G_{\widetilde{f}}$. On déduit de $F(1, x, y, 1)=\left(y^{\alpha}, P(1, x, y, 1), y^{\delta}, Q(1, x, y, 1)\right)$ que pour tous les points de $\left\{|y| \leq|x|^{N},|x|+|y| \leq \varepsilon\right\}$,

$$
\begin{aligned}
G(x, y) & =\rho_{+}^{-1} G_{\widetilde{f}}\left(y^{\beta}, P, 1, y^{\delta}, Q\right) \\
& =\rho_{+}^{-1} G_{\widetilde{f}}\left(\frac{y^{\beta}}{P}, 1, \frac{y^{\delta}}{Q}, 1\right)+\rho_{+}^{-1}(a \log |P|+b \log |Q|) \\
& \geq \rho_{+}^{-1} C_{1}+\rho_{+}^{-1}(a \log |P|+b \log |Q|) \\
& \geq c \log |x|+C_{5} \geq c \max \{\log |x|, \log |y|\}+C_{5},
\end{aligned}
$$


ce qui achève la démonstration.

\subsection{Exemples.}

Nous exhibons ici une famille d'applications polynomiales pour lesquelles le support du courant de Green est strictement inclus dans l'ensemble de Julia, ces applications ne sont donc pas normales (cf corollaire 2.7). Plus précisément, il y a un disque holomorphe isolé dans l'ensemble de Julia que le courant de Green ne peut pas charger (cf théorème 2.8). Ce phénomène est surprenant pour des applications qui ne sont pas des produits croisés.

Proposition 5.7. Considérons

$$
f:(z, w) \in \mathbb{C}^{2} \mapsto\left(z\left(w^{2}-\lambda^{2}\right), w^{2}\left(z w^{p}+Q(w)\right)\right) \in \mathbb{C}^{2},
$$

où $Q$ est un polynôme de degré $m \leq p$ et $|\lambda|>1$. On note encore $f$ l'extension à $\mathbb{P}^{1} \times \mathbb{P}^{1}$ qui est algébriquement stable. Soit $a=([0: 1],[1$ : $0]) \in I_{f}$. On note $\Omega_{a}$ l'ensemble des points attirés par a sous l'itération de $f$.

Alors il existe $r_{0}>0$ tel que

$$
\left\{(z, w) \in \mathbb{C}^{2} / 0<|z|<r_{0}^{-1} \text { et }|w|<r_{0}^{2}\right\} \subset \Omega_{a} .
$$

Le disque $\{0\} \times \Delta\left(r_{0}^{2}\right)$ est isolé dans l'ensemble de Julia $J_{f}$. En particulier Supp $T$ est strictement contenu dans $J_{f}$.

La proposition est une conséquence immédiate du lemme suivant.

Lemme 5.8. Considérons pour $\varepsilon, r>0$,

$$
W_{\varepsilon}(r):=\left\{(z, w) \in \mathbb{C}^{2} / \varepsilon<|z|<r^{-1} \text { et }|w|<r^{2}\right\} .
$$

Fixons $t, t^{\prime} \in \mathbb{R}$ tels que $1<t<|\lambda|^{2}<t^{\prime}$. Alors il existe $r_{0}>0$ tel que $\forall \varepsilon>0, \forall 0<r<r_{0}$,

$$
f\left(W_{\varepsilon}(r)\right) \subset W_{t \varepsilon}\left(r / t^{\prime}\right) .
$$

Démonstration. On fixe $r_{0}>0$ assez petit pour que $|\lambda|^{2}-r_{0}^{2} \geq t,|\lambda|^{2}+$ $r_{0}^{2} \leq t^{\prime}$ et $(1+M)\left(t^{\prime}\right)^{2} r_{0}<1$, où $M=\sup _{|w| \leq 1}|Q(w)|$. On a alors, pour $0<r<r_{0},(z, w) \in W_{\varepsilon}(r)$ et $\left(z_{1}, w_{1}\right)=f(z, w)$, les inégalités

$$
\begin{aligned}
& \left|z_{1}\right| \geq\left(\left|\lambda^{2}\right|-r^{2}\right)|z|>t \varepsilon \\
& \left|z_{1}\right| \leq\left(|\lambda|^{2}+r^{2}\right)|z|<\frac{|\lambda|^{2}+r^{2}}{r}<\frac{t^{\prime}}{r} \\
& \left|w_{1}\right| \leq|w|^{2}\left(|z||w|^{p}+|Q(w)|\right) \leq r^{4} \frac{1+M}{r}<\left(\frac{r}{t^{\prime}}\right)^{2},
\end{aligned}
$$

d'où le résultat.

Notons que le point $a=([0: 1],[1: 0])$ est un point d'indétermination, l'application $f$ n'est donc pas normale. 


\section{Produits Croisés}

Soit $A \in \mathcal{M}_{2}(\mathbb{N})$ et $f \in \mathcal{M}_{A}$ une méromorphe dominante de $\mathbb{P}^{1} \times \mathbb{P}^{1}$ de bidegrés $(\alpha, \beta)$ et $(\gamma, \delta)$. Nous avons jusqu'à présent laissé de côté certains cas particuliers.

Lorsque $\beta=\gamma=0$ et $\alpha, \delta \geq 2, f$ est holomorphe et sa dynamique s'étudie séparément sur chacun des facteurs. Lorsque $\alpha=\delta=0$ et $\beta \gamma \geq 2, f$ est également holomorphe et $f^{2}$ est du type précédent.

Lorsque $\beta \gamma=0$ et $\alpha=1$ (ou $\delta=1$ ), $f$ est semi-linéaire et sa dynamique se ramène essentiellement à de la dynamique unidimensionnelle.

Il reste donc à considérer le cas $\beta=0, \gamma \geq 1$ et $\alpha, \delta \geq 2$ (le cas $\gamma=0$, $\beta \geq 1$ et $\alpha, \delta \geq 2$ se traite symétriquement). Ce type d'applications a été étudié par plusieurs auteurs (voir [He 96], [Jo 99], [Se 97]). Notre point de vue est à rapprocher de celui de Jonsson [Jo 99] dont nous nous sommes très largement inspirés. Il considère des produits croisés polynomiaux de $\mathbb{C}^{2}$ qui admettent une extension holomorphe dans $\mathbb{P}^{2}$ et dispose dans ce cas d'une mesure mélangeante à support compact dans $\mathbb{C}^{2}$ contruite par FornaessSibony [F-S 95]. Nous construisons ici une mesure mélangeante associée à n'importe quel produit croisé polynomial de $\mathbb{C}^{2} f=(P(z), Q(z, w))$ qui n'est pas semi-linéaire. En particulier le degré en $w$ peut chuter et le support de la mesure peut être non borné dans $\mathbb{C}^{2}$. On ne peut donc pas réaliser $f$ comme une application continument fibrée au dessus de $J_{P}$, contrairement à la situation étudiée dans [Jo 00]. Il est possible d'étudier également les produits croisés rationnels, nous le ferons dans un prochain article.

Comme précedemment, on notera $\|(z, w)\|:=|z|+|w|$ si $(z, w) \in \mathbb{C}^{2}$. De même, pour $\zeta \in \mathbb{C},\|\zeta\|:=\|(1, \zeta)\|=1+|\zeta|$.

\subsection{Mesure mélangeante.}

Théorème 6.1. Soit $f:(z, w) \in \mathbb{C}^{2} \mapsto(P(z), Q(z, w)) \in \mathbb{C}^{2}$, où $P$ et $Q$ sont des polynômes tels que $\operatorname{deg} P=\alpha \geq 2, \operatorname{deg}_{w} Q=\delta \geq 2$. Soit $A(z)$ le coefficient de $w^{\delta}$. On note encore $f$ l'extension méromorphe à $\mathbb{P}^{1} \times \mathbb{P}^{1}$.

(i) La suite de courants $\alpha^{-j}\left(f^{j}\right)^{*}\left(\omega_{1}\right)$ converge vers un courant $T_{P} \in$ $\mathcal{T}\left(\mathbb{P}^{1} \times \mathbb{P}^{1}\right)$ cohomologue à $\omega_{1}$ qui vérifie

$$
f^{*} T_{P}=\alpha \cdot T_{P} \text { et Supp } T_{P}=J_{P} \times \mathbb{P}^{1},
$$

où $J_{P}$, l'ensemble de Julia de $P$, est un compact de $\mathbb{C}$. Le courant $T_{P}$ admet un potentiel partout continu.

(ii) La suite de fonctions $g_{j}(z, w)=\delta^{-j} \log \left\|Q^{j}(z, w)\right\|$ converge ponctuellement sur $K_{P} \times \mathbb{C}$ vers une fonction $g$ s.c.s. et localement bornée sur $K_{P} \times \mathbb{C}$ telle que

$$
g \circ f=\delta \cdot g
$$

où $K_{P}$ désigne l'ensemble de Julia rempli de $P$.

(iii) La suite de fonctions $\varphi_{j}(z)=\sum_{l=0}^{j-1} \log \left|A \circ P^{l}(z)\right|$ converge ponctuellement sur $K_{P}$ vers une fonction $\varphi$ s.c.s. telle que $\varphi \in L^{1}\left(\mu_{P}\right)$, où $\mu_{P}$ désigne la mesure de Green du polynôme $P$.

(iv) Lorsque $z$ est fixé dans $K_{P} \backslash\{\varphi=-\infty\}$, la fonction $w \mapsto g(z, w)$ est continue, sous-harmonique dans $\mathbb{C}$. C'est la fonction de Green avec pôle à l'infini du compact $K_{z}:=\{w \in \mathbb{C} / g(z, w)=0\}$. Elle admet 
le développement asymptotique

$$
g(z, w)=\log |w|+\varphi(z)+o_{z}(1) .
$$

Remarque 6.2. Notons que $(z, w) \in K_{P} \times \mathbb{C} \mapsto g(z, w)$ n'est, en général, pas continue(voir proposition 6.5).

\section{Démonstration.}

i) Le premier facteur est indépendant de $w$ et induit une application holomorphe $P: \mathbb{P}^{1} \rightarrow \mathbb{P}^{1}$ pour laquelle on sait construire une mesure de probabilité $\mu_{P}$ telle que $P^{*} \mu_{P}=\alpha \cdot \mu_{P}$ et qui admet un potentiel continu. Le courant $T_{P}=p_{1}^{*} \mu_{P}$ a toutes les propriétés annoncées, où $p_{1}: \mathbb{P}^{1} \times \mathbb{P}^{1} \rightarrow \mathbb{P}^{1}$ désigne la projection sur le premier facteur.

ii) Il existe $C>0$ telle que $\|Q(z, w)\| \leq C\|z\|^{\gamma}\|w\|^{\delta}$, où $\gamma=\operatorname{deg}_{z} Q$. On en déduit $g_{j+1}(z, w) \leq g_{j}(z, w)+\delta^{-(j+1)}\left(\log C+\gamma \log \left\|P^{j}(z)\right\|\right)$. Comme $K_{P}$ est un compact complètement invariant par $P$, on obtient sur $K_{P} \times \mathbb{C}$ une majoration $g_{j+1} \leq g_{j}+\delta^{-j} C^{\prime}$. La suite $\left(g_{j}\right)$ est donc une suite "quasidécroissante" de fonctions psh non-négatives, elle converge ainsi ponctuellement vers une fonction $g$ s.c.s. localement bornée sur $K_{P} \times \mathbb{C}$. La relation fonctionnelle résulte de ce que $g_{j} \circ f=\delta \cdot g_{j+1}$.

iii) On calcule par récurrence $f^{j}(z, w)=\left(P^{j}(z), Q^{j}(z, w)\right)$, où $Q^{j}$ est de terme dominant $A_{j}(z) w^{\delta^{j}}$ avec

$$
A_{j}(z)=\prod_{l=0}^{j-1}\left(A \circ P^{l}(z)\right)^{\delta^{j-l-1}} .
$$

La suite

$$
\varphi_{j}(z)=\frac{1}{\delta^{j}} \log \left|A_{j}(z)\right|=\sum_{l=0}^{j-1} \frac{1}{\delta^{l+1}} \log \left|A \circ P^{l}(z)\right|
$$

est "quasi-décroissante" sur le compact $K_{P}$ i.e. $\varphi_{j+1} \leq \varphi_{j}+\log C / \delta^{j}$ pour une constante $C>0$, car $A$ est bornée sur $K_{P}$ qui est totalement invariant. Elle converge donc vers une fonction $\varphi$ s.c.s. Cette fonction $\varphi$ n'est pas identiquement $-\infty$ puisque $P$ admet une infinité de points périodiques dont le cycle ne rencontre pas $A^{-1}(0)$. Enfin le théorème de convergence monotone donne

$$
\begin{aligned}
\int \varphi d \mu_{P} & =\lim _{j \rightarrow \infty} \int \varphi_{j} d \mu_{P} \\
& =\sum_{l \geq 0} \frac{1}{\delta^{l+1}}<\left(P^{l}\right)^{*} \log |A|, \mu_{P}> \\
& =\frac{1}{\delta-1} \int \log |A| d \mu_{P}>-\infty
\end{aligned}
$$

donc $\varphi \in L^{1}\left(\mu_{P}\right)$.

iv) Il existe une constante $C>0$ t.q. pour tout $(z, w) \in K_{P} \times \mathbb{C}$,

$$
|\|Q(z, w)\|-| A(z)\left|\cdot\|w\|^{\delta}\right| \leq C\|w\|^{\delta-1} .
$$

On en déduit $\forall j \in \mathbb{N}$,

$$
\left|g_{j+1}-g_{j}-\frac{1}{\delta^{j+1}} \log \right| A \circ P^{j}|| \leq \frac{1}{\delta^{j+1}} \log \left(1+\frac{C}{\left\|Q^{j}\right\| \cdot\left|A \circ P^{j}\right|}\right) .
$$


En minorant $\left\|Q^{j}\right\| \geq 1$ et en sommant l'inégalité précédente, on obtient $\forall j \in \mathbb{N}, \forall(z, w) \in K_{P} \times \mathbb{C}$,

$$
\left|g(z, w)-g_{j}(z, w)-\left(\varphi(z)-\varphi_{j}(z)\right)\right| \leq \sum_{l \geq j} \frac{1}{\delta^{l+1}} \log \left(1+\frac{C}{\left|A \circ P^{l}\right|}\right) .
$$

Fixons $z \in K_{P} \backslash\{\varphi=-\infty\}$. Comme la série du membre de droite converge, la suite $\left(g_{j}\right)$ converge uniformément vers $g$ sur $\{z\} \times \mathbb{C}$ et $w \mapsto g(z, w)$ est continue sur $\mathbb{C}$. On obtient également $\forall(z, w) \in K_{P} \times \mathbb{C}$,

$$
|g(z, w)-\log \|w\|-\varphi(z)| \leq \psi(z, w),
$$

avec

$$
\psi(z, w):=\sum_{l \geq 0} \frac{1}{\delta^{l+1}} \log \left(1+\frac{C}{\left|A \circ P^{l}\right|}\right) .
$$

Il s'ensuit, par convergence dominée,

$$
\lim _{w \rightarrow \infty} \psi(z, w)=\sum_{l \geq 0} \frac{1}{\delta^{l+1}} \lim _{w \rightarrow \infty} \log \left(1+\frac{C}{\left|A \circ P^{l}\right| \cdot\left\|Q^{l}\right\|}\right)=0,
$$

d'où $g(z, w)=\log \|w\|+\varphi(z)+o_{z}(1)$. Ainsi $K_{z}=\{w \in \mathbb{C} / g(z, w)=0\}$ est un compact de $\mathbb{C}, w \mapsto g(z, w)$ est sa fonction de Green avec pôle à l'infini et $K_{z}$ est de capacité logarithmique $e^{-\varphi(z)}$.

Théorème 6.3. Soit $f=(P(z), Q(z, w))$ vérifiant les hypothèses du théorème 6.1. On note $\mu_{P}$ la mesure de Green de $P$ et $\mu_{z}:=\Delta_{w} g(z, w)$.

(i) Le courant $\mu:=d d^{c}\left(g \cdot T_{P}\right)$ est une mesure de probabilité invariante dans $\mathbb{C}^{2}$ qui vérifie $f^{*} \mu=\alpha \delta \cdot \mu$. Elle agit sur une forme test $\chi$ par

$$
<\mu, \chi>=\int\left(\int \chi(z, w) d \mu_{z}(w)\right) d \mu_{P}(z) .
$$

(ii) La mesure $\mu$ ne charge pas les ensembles pluripolaires. Elle est mélangeante.

(iii) Le support de $\mu$ vérifie

$$
\text { Supp } \mu=\overline{\bigcup_{z \in J_{P} \backslash\{\varphi=-\infty\}}\{z\} \times \partial K_{z}} .
$$

Il est compact dans $\mathbb{C}^{2}$ si et seulement si $A^{-1}(0) \cap J_{P}=\emptyset$.

(iv) Les fonctions $\log ^{+}\left\|(D f)^{ \pm 1}\right\|$ sont dans $L^{1}(\mu)$. Ainsi $\mu$ admet deux exposants de Lyapunov $\lambda^{\prime}, \lambda^{\prime \prime}$. Ils sont donnés par

$$
\lambda^{\prime}=\lambda_{P}=\log \alpha+\sum_{P^{\prime}\left(z_{i}\right)=0} g_{P}\left(z_{i}\right) \geq \log \alpha>0
$$

et

$$
\lambda^{\prime \prime}=\log \delta+\int \sum_{\frac{\partial Q}{\partial w}\left(z, w_{i}(z)\right)=0} g\left(z, w_{i}(z)\right) d \mu_{P}(z) \geq \log \delta>0 .
$$


Remarque 6.4. La positivité des exposants de Lyapunov assure que les points périodiques répulsifs s'équidistribuent selon $\mu$. Lorsque le support de $\mu$ est un compact de $\mathbb{C}^{2}$, il suffit pour le montrer de reprendre les arguments donnés par Briend-Duval (voir [Br 97] et [Br-D 99]) dans le cas des endomorphismes de $\mathbb{P}^{k}$, f étant holomorphe au voisinage du support de $\mu$. La situation est plus délicate lorsque le support de $\mu$, non compact, contient des points d'indétermination à l'infini. Nous détaillerons ce résultat dans un prochain article.

\section{Démonstration.}

i) Le courant $\mu=d d^{c}\left(g \cdot T_{P}\right)$ est bien défini dans $\mathbb{C}^{2}$ car $g$ est localement bornée sur le support de $T_{P}$. C'est une mesure positive puisque $g_{j}$ est psh dans $\mathbb{C}^{2}$ et $\mu=\lim d d^{c}\left(g_{j} \cdot T_{P}\right)$. L'équation fonctionnelle $f^{*} \mu=\alpha \delta \cdot \mu$ résulte de celles satisfaites par $g$ et $T_{P}$. Enfin la masse de $\mu$ dans $\mathbb{C}^{2}$ est

$$
\|\mu\|_{\mathbb{C}^{2}}=\int_{z \in \mathbb{C}}\left(\int_{w \in \mathbb{C}} \Delta_{w} g(z, w)\right) d \mu_{P}(z)=1,
$$

car $\varphi \in L^{1}\left(\mu_{P}\right)$ et $\int_{w} \Delta_{w} g(z, w)=1$ pour $z \in J_{P} \backslash\{\varphi=-\infty\}$ (cf 6.1.iv).

ii) La proposition 4.12 assure que $\mu$ ne charge pas les ensembles pluripolaires. Comme le degré topologique de $f$ est $d_{t}(f)=\alpha \delta>\lambda_{1}(f)=$ $\max (\alpha, \delta)$, le résultat d'équidistribution de Russakovskii-Shiffman (voir théorème 4.6) et le lemme 4.11 montrent que $f$ est mélangeante

iii) La formule annoncée pour le support est une conséquence de (17). Lorsque $A^{-1}(0) \cap J_{P}=\emptyset$, la fonction $\varphi$ est uniformément minorée sur $J_{P}$ et on déduit de (16) que $g(z, w)-\log \|w\| \geq C_{1}$ pour une constante $C_{1}>0$. Cela prouve la compacité de $\operatorname{Supp}(\mu)$.

Si $A^{-1}(0) \cap J_{P} \neq \emptyset$ alors $\{\varphi=-\infty\} \cap J_{P} \neq \emptyset$. Soit $\left(x_{j}\right)$ une suite de points de $J_{P} \backslash\{\varphi=-\infty\}$ qui converge vers $x$ tel que $\varphi(x)=-\infty$. On a $\varphi\left(x_{j}\right) \rightarrow-\infty$ donc la suite de compacts $\partial K_{x_{j}} \subset \operatorname{Supp}(\mu)$ est non bornée -ils sont de capacité logarithmique $\exp \left(-\varphi\left(x_{j}\right)\right)$ - et $\operatorname{Supp}(\mu)$ n'est pas borné.

iv) Pour montrer que les fonctions $\log ^{+}\left\|(D f)^{ \pm 1}\right\|$ sont dans $L^{1}(\mu)$, il suffit de montrer que $\log ^{+}|w| \in L^{1}(\mu)$. Or pour $z \in J_{P} \backslash\{\varphi=-\infty\}$, la formule de représentation de Riesz s'écrit

$$
\forall \zeta \in \mathbb{C}, \int_{\mathbb{C}} \log |w-\zeta| \Delta_{w} g(z, w)=g(z, \zeta)-\varphi(z) .
$$

On peut approcher $\log ^{+}|w|$ par $d_{j}^{-1} \log \left|B_{j}(w)\right|$, où les $B_{j}$ sont des polynômes de degré $d_{j}$ dont les racines sont sur $\partial D=\{|w|=1\}$. La formule de Riesz donne alors

$$
\int_{\mathbb{C}} \log ^{+}|w| \Delta_{w} g(z, w) \leq \sup _{\zeta \in \partial D} g(z, \zeta)-\varphi(z) .
$$

Comme $g$ est majorée sur $J_{P} \times \partial D$, on en déduit

$$
\int_{\mathbb{C}^{2}} \log ^{+}|w| d \mu(z, w) \leq \int_{\mathbb{C}}-\varphi(z) d \mu_{P}(z)+C_{2}<+\infty,
$$

ainsi $\log ^{+}|w| \in L^{1}(\mu)$.

L'application $f$ admet donc deux exposants de Lyapunov relatifs à la mesure ergodique $\mu$. Ce sont deux réels $\lambda_{1} \geq \lambda_{2}$ caractérisés par l'existence, 
pour $\mu$-presque tout $m \in \mathbb{C}^{2}$ d'un sous-espace $E_{2}(m)$ de $\mathbb{C}^{2}$ tel que

$$
\begin{aligned}
& \lim _{j \rightarrow+\infty} \frac{1}{j} \log \left\|D f^{j} \cdot v\right\|=\lambda_{2}, \forall v \in E_{2}(m) \backslash\{0\}, \\
& \lim _{j \rightarrow+\infty} \frac{1}{j} \log \left\|D f^{j} \cdot v\right\|=\lambda_{1}, \forall v \in \mathbb{C}^{2} \backslash E_{2}(m) .
\end{aligned}
$$

On a de plus ( $\mathrm{cf}[\mathrm{Y}$ 95])

$$
\Lambda=\lambda_{1}+\lambda_{2}=\int \log |\operatorname{det} D f| d \mu .
$$

Puisque $f$ envoie une droite verticale $\{z\} \times \mathbb{C}$ sur la droite verticale $\{P(z)\} \times$ $\mathbb{C}$, l'un des exposants de Lyapunov, notons le $\lambda^{\prime}$, est égal à l'exposant de Lyapunov $\lambda_{P}$ de $P$ relatif à la mesure $\mu_{P}$ qui est ergodique pour $P$. La formule annoncée pour $\lambda^{\prime}$ est alors une conséquence simple de la formule de Riesz pour $g_{P}$, la fonction de Green de $P(\mathrm{cf}[\mathrm{P} 85])$. Il reste à calculer $\lambda^{\prime \prime}=\Lambda-\lambda_{P}$. On déduit de (17) et (18)

$$
\lambda^{\prime \prime}=\Lambda-\lambda_{P}=\int\left(\int \log \left|\frac{\partial Q}{\partial w}(z, w)\right| d \mu_{z}(w)\right) d \mu_{P}(z) .
$$

Or pour tout $z \in J_{P} \backslash\{\varphi=-\infty\}, g(z, w)=\varphi(z)+\int \log |\zeta-w| \mu_{z}(\zeta)$, et le terme dominant de $Q_{z}(w)=Q(z, w)$ est $A(z) w^{\delta}$. Si $w_{1}(z), \ldots, w_{\delta-1}(z)$ sont les racines de $\partial Q_{z} / \partial w(w)=0$ (comptées avec multiplicité), on obtient donc

$$
\int \log \left|\frac{\partial Q}{\partial w}(z, w)\right| d \mu_{z}(w)=\log \delta+\log |A(z)|+\sum_{i=1}^{\delta-1}\left(g\left(z, w_{i}(z)\right)-\varphi(z)\right) .
$$

Enfin l'égalité $(\delta-1) \int \varphi d \mu_{P}=\int \log |A| d \mu_{P}$ (cf démonstration de 6.1.iii) fournit la formule désirée pour $\lambda^{\prime \prime}$.

\subsection{Exemples.}

Nous précisons dans ce paragraphe la dynamique d'une classe particulière de produits croisés polynomiaux. Cela fournit en particulier des exemples d'applications polynomiales de $\mathbb{C}^{2}$ pour lesquelles l'ensemble $K_{f}$ des points d'orbite positive bornée n'est pas fermé.

On considère $f:(z, w) \in \mathbb{C}^{2} \rightarrow\left(P(z), A(z) w^{\delta}\right) \in \mathbb{C}^{2}$, avec $\delta \geq 2, \operatorname{deg} P=$ $\alpha \geq 2$. Comme précédemment $\varphi(z)=\sum_{l \geq 0} \frac{1}{\delta^{l+1}} \log \left|A \circ P^{l}(z)\right|$. On vérifie aisément les assertions suivantes.

- La fonction $g: K_{P} \times \mathbb{C} \rightarrow \mathbb{R}$ est donnée par $g(z, w)=\max (\varphi(z)+$ $\log |w|, 0)$.

- $K_{z}=\left\{w \in \mathbb{C} /|w| \leq e^{-\varphi(z)}\right\}$.

- Le second exposant de Lyapunov est $\lambda^{\prime \prime}=\log \delta$.

Il est possible de décrire précisément certains aspects de la dynamique en discutant selon que $A^{-1}(0)$ rencontre ou non $J_{P}\left(\right.$ resp. $\left.K_{P}^{\circ}\right)$. Nous ne mentionnerons que la

Proposition 6.5. Lorsque $A^{-1}(0) \cap J_{P} \neq \emptyset$, l'ensemble $\{\varphi=-\infty\} \cap J_{P}$ est dense dans $J_{P}$. La fonction $g_{\mid J_{P} \times \mathbb{C}}$ est partout discontinue. L'ensemble $K_{f}=\left\{(z, w) \in \mathbb{C}^{2} /\left(f^{j}(z, w)\right)_{j \geq 0}\right.$ bornée $\}$ des points d'orbite positive bornée n'est pas fermé. Son adhérence contient $J_{P} \times \mathbb{C}$. 
Démonstration. Soit $x$ un point de $J_{P}$ tel que $A(x)=0$. Les préimages de $x$ par $P^{j}$ sont denses dans $J_{P}$ et appartiennent à $\cup_{j \geq 1}\left\{\varphi_{j}=-\infty\right\} \subset$ $\{\varphi=-\infty\}$. La fonction $\varphi_{\mid J_{P}}$ ne peut donc pas être continue aux points de $J_{P} \backslash\{\varphi=-\infty\}$. Par suite, $g$ est nulle sur une famille de droites $\{z\} \times \mathbb{C}$ dense dans $J_{P} \times \mathbb{C}$ et $\left.g\right|_{J_{P} \times \mathbb{C}}$ est partout discontinue.

L'ensemble $K_{f}$ est un sous-ensemble de $K_{P} \times \mathbb{C}$. Si $\log |w|>-\varphi(z)$, l'équation fonctionnelle $g \circ f=\delta \cdot g$ assure que $\left|w_{j}\right| \rightarrow \infty$, l'orbite de $(z, w)$ est attirée par $\left\{w_{0}=0\right\}$, donc $(z, w) \notin K_{f}$.

Si $z \in K_{P}$ et $\varphi(z)+\log |w|<0$,

$$
\frac{1}{\delta^{j}} \log \left|w_{j}\right|=\log |w|+\sum_{l=0}^{j-1} \frac{1}{\delta^{l+1}} \log \left|A \circ P^{l}(z)\right|
$$

est strictement négatif pour $j$ assez grand. Donc $\left|w_{j}\right| \rightarrow 0$ et $(z, w) \in K_{f}$.

Ainsi $K_{f} \cap\left(J_{P} \times \mathbb{C}\right)$ est strictement inclus dans $J_{P} \times \mathbb{C}$ et contient une famille dense de droites $\{z\} \times \mathbb{C}$, il n'est donc pas fermé.

\section{Applications birationnelles de $\mathbb{P}^{1} \times \mathbb{P}^{1}$}

\subsection{Généralités.}

Définition 7.1. Une application $f: \mathbb{P}^{1} \times \mathbb{P}^{1} \rightarrow \mathbb{P}^{1} \times \mathbb{P}^{1}$ rationnelle est dite birationnelle s'il existe une application rationnelle $g: \mathbb{P}^{1} \times \mathbb{P}^{1} \rightarrow \mathbb{P}^{1} \times \mathbb{P}^{1}$ telle que $f \circ g$ est l'identité, en tant qu'application rationnelle. Dans ce cas on notera $f^{-1}$ l'application $g$.

On se restreindra dans toute la suite au cas où la plus grande valeur propre $\rho_{+}$de la matrice des degrés de $f$ satisfait $\rho_{+}>1$. Le cas d'égalité ne se produit que lorsque $f$ est un automorphisme linéaire de $\mathbb{P}^{1} \times \mathbb{P}^{1}$ ou lorsque $f$ est conjuguée à $(z, w) \mapsto\left(a z+b, R_{1}(z) w+R_{2}(z)\right)$ avec $a \neq 0$, et $R_{1}, R_{2} \in \mathbb{C}(z)$.

Nous utiliserons à plusieurs reprises l'observation suivante.

Observation 7.2. Soit $f$ une application birationnelle de $\mathbb{P}^{1} \times \mathbb{P}^{1}$. Alors

- $f$ réalise un biholomorphisme de $\mathbb{P}^{1} \times \mathbb{P}^{1} \backslash \mathcal{C}^{+}$sur $\mathbb{P}^{1} \times \mathbb{P}^{1} \backslash \mathcal{C}^{-}$,

- $f\left(\mathcal{C}^{+}\right)=I^{-}$,

où $\mathcal{C}^{+}, \mathcal{C}^{-}$désignent les ensembles critiques de $f, f^{-1}$.

Proposition 7.3. Soit $A \in \mathcal{M}_{2}(\mathbb{N})$ et $f \in \mathcal{M}_{A}$ une application birationnelle de $\mathbb{P}^{1} \times \mathbb{P}^{1}$. Alors $f^{-1} \in \mathcal{M}_{t}$, où ${ }^{t} A$ désigne la matrice transposée de $A$. En particulier $\lambda_{1}(f)=\lambda_{1}\left(f^{-1}\right)$.

Démonstration. Soit $\omega^{\prime}$ et $\omega^{\prime \prime}$ deux formes lisses fermées de bidegré $(1,1)$ sur $\mathbb{P}^{1} \times \mathbb{P}^{1}$. Les courants $f^{*} \omega^{\prime} \wedge \omega^{\prime \prime}$ et $\omega^{\prime} \wedge\left(f^{-1}\right)^{*} \omega^{\prime \prime}$ ne chargent pas les hypersurfaces, on a donc

$$
\begin{aligned}
\int_{\mathbb{P}^{1} \times \mathbb{P}^{1}} f^{*} \omega^{\prime} \wedge \omega^{\prime \prime} & =\int_{\mathbb{P}^{1} \times \mathbb{P}^{1} \backslash \mathcal{C}^{+}} f^{*} \omega^{\prime} \wedge \omega^{\prime \prime} \\
& =\int_{\mathbb{P}^{1} \times \mathbb{P}^{1} \backslash \mathcal{C}^{-}} \omega^{\prime} \wedge\left(f^{-1}\right)^{*} \omega^{\prime \prime} \\
& =\int_{\mathbb{P}^{1} \times \mathbb{P}^{1}} \omega^{\prime} \wedge\left(f^{-1}\right)^{*} \omega^{\prime \prime} .
\end{aligned}
$$


L'application $\left(\left[\omega^{\prime}\right],\left[\omega^{\prime \prime}\right]\right) \in H^{1,1}\left(\mathbb{P}^{1} \times \mathbb{P}^{1}, \mathbb{R}\right)^{2} \rightarrow \int_{\mathbb{P}^{1} \times \mathbb{P}^{1}} f^{*} \omega^{\prime} \wedge \omega^{\prime \prime}$ est une forme bilinéaire $\Phi_{f}$ dont l'action dans la base $\left(\left[\omega_{1}\right],\left[\omega_{2}\right]\right)$ est donnée par la matrice $A_{f}$. L'égalité ci-dessus signifie que $\Phi_{f^{-1}}$ est l'application duale de $\Phi_{f}$, on a donc $A_{f^{-1}}={ }^{t} A_{f}$.

Proposition 7.4. Soit $A \in \mathcal{M}_{2}(\mathbb{N})$ et $f \in \mathcal{M}_{A}$ une application birationnelle de $\mathbb{P}^{1} \times \mathbb{P}^{1}$. Les conditions suivantes sont équivalentes

i) $f$ est algébriquement stable ;

ii) $\lambda_{1}(f)=\rho_{+}$;

iii) $I_{\infty}^{+} \cap I_{\infty}^{-}=\emptyset$;

iv) $f^{-1}$ est algébriquement stable, où $I_{\infty}^{ \pm}=\cup_{j \geq 1} I_{f^{ \pm j}}$.

Démonstration. C'est une conséquence directe de 7.2 et 7.3.

Nous aurons besoin dans la suite de l'information suivante, un résultat similaire a été obtenu dans $\mathbb{P}^{2}$ par Diller [Di 98].

Proposition 7.5. Soit $f$ une application birationnelle algébriquement stable de $\mathbb{P}^{1} \times \mathbb{P}^{1}$ et vérifiant $\rho_{+}>1$. Soit $T^{+}$le courant de Green de $f$. Alors

$$
\nu\left(T^{+}, p\right)>0 \text { ssi } p \in I_{\infty}^{+} .
$$

Démonstration. Soit $G^{+}$un potentiel de $T^{+}$dans $\mathbb{C}^{2} \times \mathbb{C}^{2}$. La relation $G^{+}(\lambda z, \mu w)=a^{+} \log |\lambda|+b^{+} \log |\mu|+G^{+}(z, w)$ assure que $G^{+}$a un nombre de Lelong supérieur ou égal à $\min \left(a^{+}, b^{+}\right)>0$ en tout point de $\{0\} \times \mathbb{C} \cup$ $\mathbb{C} \times\{0\}$. Soit $p \in I_{f^{j}} \subset I_{\infty}^{+}$et $q \in \pi^{-1}(p)$. Soit $F$ un relèvement de $f$ dans $\mathbb{C}^{2} \times \mathbb{C}^{2}$ normalisé de telle sorte que $G^{+} \circ F=\rho_{+} \cdot G^{+}$. Comme $F^{j}(q) \in\{0\} \times \mathbb{C} \cup \mathbb{C} \times\{0\}$, on a

$$
\nu\left(G^{+}, q\right)=\frac{1}{\rho_{+}^{j}} \nu\left(G^{+} \circ F^{j}, q\right) \geq \frac{1}{\rho_{+}^{j}} \nu\left(G^{+}, F^{j}(q)\right) \geq \frac{\min \left(a^{+}, b^{+}\right)}{\rho_{+}^{j}}>0 .
$$

Ainsi $\nu\left(T^{+}, p\right)>0$.

Réciproquement, soit $p \in \mathbb{P}^{1} \times \mathbb{P}^{1} \backslash I_{\infty}^{+}$. Supposons que pour tout $j \geq 0$, $f^{j}(p) \notin \mathcal{C}_{f}$. Les nombres de Lelong sont invariants par biholomorphisme (voir [S 74]), donc on a pour tout $j \in \mathbb{N}$

$$
\nu\left(T^{+}, p\right)=\frac{1}{\rho_{+}^{j}} \nu\left(\left(f^{j}\right)^{*} T^{+}, p\right)=\frac{1}{\rho_{+}^{j}} \nu\left(T^{+}, f^{j}(p)\right) \leq \frac{C}{\rho_{+}^{j}}
$$

avec $C=\max \left\{\nu\left(T^{+}, z\right), z \in \mathbb{P}^{1} \times \mathbb{P}^{1}\right\}$. Donc $\nu\left(T^{+}, p\right)=0$.

Quitte à remplacer $f$ par un itéré on peut donc supposer que $p \in \mathcal{C}_{f}$. Soit $V$ une composante irréductible de $\mathcal{C}_{f}$ contenant $p$. Comme $T^{+}$ne charge pas $V$ (théorème 2.8.), il existe $q \in V$ tel que $\nu\left(T^{+}, q\right)=0$. De plus, il existe une constante $C_{f}>0$ telle que $\nu\left(f^{*} T^{+}, p\right) \leq C_{f} \times \nu\left(T^{+}, f(p)\right)(\mathrm{cf}[$ Fa 99] et [Ki 00]). Donc

$$
\nu\left(T^{+}, p\right)=\frac{1}{\rho_{+}} \nu\left(f^{*} T^{+}, p\right) \leq \frac{C_{f}}{\rho_{+}} \nu\left(T^{+}, f(p)\right) \leq \frac{C_{f}}{\rho_{+}} \nu\left(f^{*} T^{+}, q\right)=0,
$$

puisque $f(q)=f(p)$ est un point de $I^{-}$(cf observation 7.2). 


\subsection{Convergence vers $T^{+}$et points exceptionnels.}

Soit $f$ une application birationnelle de $\mathbb{P}^{1} \times \mathbb{P}^{1}$ algébriquement stable telle que $\rho_{+}>1$. Le but de ce paragraphe est de caractériser le convexe compact $\mathcal{K}_{f}$ des courants positifs fermés $T$ de bidegré $(1,1)$ et de masse 1 qui vérifient l'équation fonctionnelle $f^{*} T=\rho_{+} T$. Les éléments extrémaux de $\mathcal{K}_{f}$ sont $T^{+}$et certains courants d'intégration sur des courbes "exceptionnelles" de l'ensemble critique. Notre principal résultat est le théorème de convergence suivant.

Théorème 7.6. [Convergence vers $T^{+}$]

Soit $f$ une application birationnelle de $\mathbb{P}^{1} \times \mathbb{P}^{1}$ algébriquement stable t.q. $\rho_{+}>1$. Soit $T^{+}=\mathcal{L}\left(G^{+}\right)$le courant de Green de $f$. Il est cohomologue à $\omega^{+}:=a^{+} \omega_{1}+b^{+} \omega_{2}$ pour des constantes $a^{+}, b^{+}>0$.

Alors il existe un ensemble fini $\mathcal{E} x$, tel que pour tout $S \in \mathcal{T}\left(\mathbb{P}^{1} \times \mathbb{P}^{1}\right)$, les conditions suivantes sont équivalentes.

(1) Pour tout $p \in \mathcal{E} x, \nu(p, S)=0$.

(2) Il existe une constante $c>0$ ne dépendant que de la classe de cohomologie de $S$ t.q.

$$
\frac{1}{\rho_{+}^{j}}\left(f^{j}\right)^{*}(S) \longrightarrow c T^{+}
$$

De plus si $V$ est un voisinage fixé de $\mathcal{E} x$, la convergence dans (19) est uniforme dans le compact des courants de masse 1 et dont le support évite $V$.

La démonstration s'appuie sur des estimations volumiques, i.e. sur une minoration fine de la suite $\operatorname{Vol}\left(f^{j}(E)\right)$ pour un borélien $E$ fixé. Elle se situe dans l'esprit de celle initiée par Bedford-Smillie ([B-S 91]), Fornaess-Sibony ([F-S 92]) dans le cas des applications de Hénon, et de Diller ([Di 96])) dans celui des applications birationnelles de $\mathbb{P}^{2}$. Notons qu'un résultat similaire se démontre pour les applications birationnelles algébriquement stables de $\mathbb{P}^{2}$ et constitue la version aboutie des procédés de convergence développés jusqu'à présent, en particulier dans le cas des applications de Hénon.

Commençons par quelques définitions. Nous noterons $S A_{f}$ l'ensemble des points périodiques superattractifs de $f$, i.e. ceux dont les deux valeurs propres s'annulent. Cet ensemble est fini car le nombre de cycles superattractifs est majoré par le nombre de composantes irréductibles de l'ensemble critique $\mathcal{C}_{f}$. On notera $\Omega\left(S A_{f}\right)=\cup_{p \in S A_{f}} \Omega(p)$ la réunion des bassins d'attractions des cycles superattractifs.

Soit $p \in S A_{f}$ un point périodique que l'on peut supposer fixe quitte à prendre un itéré de $f$. Comme $f$ est birationnelle, il découle de la proposition 2.1. de [Fa 00] que le germe défini par $f$ en $p$ est rigide, c'est-à-dire que $\cup_{n \geq 0} \mathcal{C}_{f^{n}}$ est un germe de courbe analytique à croisements normaux. L'article [Fa 00] est consacré à la classification de ce type de germes. On en déduit que $(f, p)$ est conjuguée à l'une des formes normales suivantes.

- Classe 1:

$$
f_{1}(z, w)=\left(z^{a_{1}}, \lambda w z^{a_{2}}+P(z)\right)
$$

avec $a_{1} \geq 2, \lambda \neq 0, a_{2} \geq 1$ et $P$ est un polynôme s'annulant en 0 .

- Classe 2:

$$
f_{2}(z, w)=\left(z^{a_{1}} w^{a_{2}}, z^{a_{3}} w^{a_{4}}\right)
$$


avec $\left|a_{1} a_{4}-a_{2} a_{3}\right|=1$.

- Classe 3:

$$
\begin{aligned}
& f_{3}(z, w)=\left(\left(z^{a_{1}} w^{a_{2}}\right)^{u}\left(1+\lambda z^{b_{1}} w^{b_{2}}+P\left(z^{a_{1}} w^{a_{2}}\right)\right)^{-a_{2}}\right. \\
&\left.\left(z^{a_{1}} w^{a_{2}}\right)^{v}\left(1+\lambda z^{b_{1}} w^{b_{2}}+P\left(z^{a_{1}} w^{a_{2}}\right)\right)^{a_{1}}\right)
\end{aligned}
$$

avec $a_{1}+a_{2}, b_{1}+b_{2} \geq 1, u, v \geq 1, \lambda \neq 0$ et $\left|a_{1} b_{2}-a_{2} b_{1}\right|=1$ et $P$ est un polynôme s'annulant en 0 .

Remarque 7.7. Notons que lorsque $p \in S A_{f}$ est fixé, le germe $\left(\mathcal{C}_{f}, p\right)$ n'est pas toujours totalement invariant. Par contre $\left(\mathcal{C}_{f^{2}}, p\right)$ l'est et pour tout $j \geq 2,\left(\mathcal{C}_{f^{j}}, p\right)=\left(\mathcal{C}_{f^{2}}, p\right)$. Par la suite nous considérerons ainsi les itérés de $f^{2}$.

Définition 7.8. Soit $p \in S A_{f}$ de période $n$. On définit le réel $\rho(p)$ par

- $\rho(p)^{n}:=a_{1}$ si $(f, p)$ est de classe 1 ;

- $\rho(p)^{n}:=2^{-1}\left(a_{1}+a_{4}+\sqrt{\left(a_{1}-a_{4}\right)^{2}+4 a_{2} a_{3}}\right)$ si $(f, p)$ est de classe 2

;

- $\rho(p)^{n}:=a_{1} u+a_{2} v$ si $(f, p)$ est de classe 3.

Le réel $\rho(p)^{n}$ est le rayon spectral de l'action de $f^{n}$ sur l'espace des $\mathbb{R}$-cycles engendrés par les composantes de $\mathcal{C}_{f^{2 n}}$ contenant $p$.

Notons que $\rho(p)>1$ dans chacun des trois cas car $f$ est superattractive. Le réel $\rho(p)$ contrôle précisément la décroissance du volume $\operatorname{Vol}\left(f^{j}(E)\right)$ lorsque $E \subset \Omega(p)$ (voir proposition 7.12).

Lemme 7.9. Soit $f$ une application birationnelle de $\mathbb{P}^{1} \times \mathbb{P}^{1}$ algébriquement stable t.q. $\rho_{+}>1$ et $p \in S A_{f}$ un point périodique. Alors $\rho(p) \leq \rho_{+}$.

De plus $\rho(p)=\rho_{+}$ssi il existe un $\mathbb{R}$-cycle $[V]$ contenant $p$, cohomologue $\grave{a} T^{+}$et à coefficients réels positifs, t.q. $f^{*}[V]=\rho_{+}[V]$.

Démonstration. Notons $n$ la période du point $p$. Puisque $f$ n'est pas un produit croisé (nous avons supposé $\rho_{+}>1$ ), il existe une constante $c>0$ telle que $\forall S \in \mathcal{T}\left(\mathbb{P}^{1} \times \mathbb{P}^{1}\right),\left\|\left(f^{j}\right)^{*}(T)\right\| \leq c \rho_{+}^{j}\|T\|$.

Lorsque $f$ est de classe 1 , le germe $\mathcal{C}_{f^{n}}$ est irréductible en $p$. Soit $V$ la composante irréductible (globale) de $\mathcal{C}_{f^{n}}$ contenant $p$. Au voisinage de $p$,

$$
\left(\left(f^{n}\right)^{*}[V], p\right)=\left(\rho(p)^{n}[V], p\right) .
$$

Globalement on obtient donc $\left(f^{n j}\right)^{*}[V] \geq \rho(p)^{n j}[V]$ pour tout $j \in \mathbb{N}$, d'où $\rho(p) \leq \rho_{+}$. Lorsque $\rho(p)=\rho_{+}$, on a $\left(f^{n}\right)^{*}[V] \geq \rho_{+}^{n}[V]$, ce qui force $\left(f^{n}\right)^{*}[V]=\rho_{+}^{n}[V]$. On conclut en remarquant que

$$
f^{*}\left(\sum_{k=0}^{n-1} \rho_{+}^{n-k}\left(f^{k}\right)^{*}[V]\right)=\rho_{+}\left(\sum_{k=0}^{n-1} \rho_{+}^{n-k}\left(f^{k}\right)^{*}[V]\right) .
$$

Si $f$ est de classe 2 ou 3 , notons $V_{1}$ et $V_{2}$ les deux composantes irréductibles locales du germe défini par $\mathcal{C}_{f} 2 n$ au voisinage de $p$. Supposons que $V_{1}$ et $V_{2}$ appartiennent à la même courbe irréductible $V \subset \mathbb{P}^{1} \times \mathbb{P}^{1}$. Après un nombre fini d'éclatements de points au dessus de $I_{f 2 n}$, la transformée stricte de $V$ devient une courbe rationnelle lisse (voir e.g. [Be 83]), ce qui est contradictoire. Donc $V_{1}$ et $V_{2}$ définissent globalement deux courbes irréductibles distinctes. 
Définissons le cycle $[V]:=a_{3}\left[V_{1}\right]+\left(\rho(p)^{n}-a_{1}\right)\left[V_{2}\right]$ si $f$ est de classe

2 (le vecteur $\left(a_{3}, \rho(p)^{n}-a_{1}\right)$ est propre pour la matrice $\left[\begin{array}{ll}a_{1} & a_{3} \\ a_{2} & a_{4}\end{array}\right]$ ), et $[V]:=a_{1}\left[V_{1}\right]+a_{2}\left[V_{2}\right]$ si $f$ est de classe 3 . Ce sont des $\mathbb{R}$-cycles à coefficients positifs. Pour tout $j \in \mathbf{N}$, on a localement

$$
\left(\left(f^{n j}\right)^{*}[V], p\right)=\left(\rho(p)^{n}[V], p\right) .
$$

Comme précédemment, on en déduit $\rho(p) \leq \rho_{+}$et, lorsque $\rho(p)=\rho_{+}$, $\left(f^{n}\right)^{*}[V]=\rho_{+}[V]$.

Définition 7.10. [Points exceptionnels]

Soit $A \in \mathcal{M}_{2}(\mathbb{N})$ avec $\beta \gamma \neq 0$, et $f \in \mathcal{M}_{A}$ une application birationnelle algébriquement stable de $\mathbb{P}^{1} \times \mathbb{P}^{1}$ qui n'est pas linéaire (i.e. $\rho_{+}>1$ ).

Un point $p \in S A_{f}$ est dit exceptionnel si $\rho(p)=\rho_{+}$. On notera $\mathcal{E} x$ l'ensemble des points exceptionnels de $f$.

Le théorème suivant donne une estimation précise de la décroissance de $\operatorname{Vol}\left(f^{j}(E)\right)$ lorsque $j$ tend vers l'infini. C'est le point clé de la démonstration du résultat de convergence des courants (théorème 7.6).

Théorème 7.11. [Estimations volumiques]

Soit $f$ une application birationnelle de $\mathbb{P}^{1} \times \mathbb{P}^{1}$ algébriquement stable t.q. $\rho_{+}>1$. On note $\Omega(p)$ le bassin d'attraction d'un point $p \in S A_{f}$. Pour $r>0$ assez petit, on définit $\Omega_{r}(p):=\{z \in \Omega(p) / \operatorname{dist}(z, \partial \Omega(p))>r\}$.

Fixons $\lambda>1$ et $\rho_{0}>1$ t.q. $\rho>\rho_{0} \geq \sup \left\{\rho(p), p \in S A_{f} \backslash \mathcal{E} x\right\}$. Alors, il existe $C_{1}, C_{2}>0$ t.q.

(1) pour tout borélien $E \subset \mathbb{P}^{1} \times \mathbb{P}^{1} \backslash \bigcup_{p \in S A_{f}} \Omega(p)$, et $\forall j \geq 0$,

$$
\operatorname{Vol}\left(f^{j}(E)\right) \geq\left(C_{1} \operatorname{Vol}(E)\right)^{C_{2} \lambda^{j}}
$$

(2) pour tout borélien $E \subset \bigcup_{p \in S A_{f} \backslash \mathcal{E} x} \Omega_{r}(p)$, et $\forall j \geq 0$,

$$
\operatorname{Vol}\left(f^{j}(E)\right) \geq\left(C_{1} x(E)\right)^{C_{2} \rho_{0}^{j}} ;
$$

(3) pour tout borélien $E \subset \bigcup_{p \in \mathcal{E} x} \Omega_{r}(p)$, et $\forall j \geq 0$,

$$
\operatorname{Vol}\left(f^{j}(E)\right) \geq\left(C_{1} x(E)\right)^{C_{2} \rho_{+}^{j}} ;
$$

avec $-x(E) \log x(E)=\operatorname{Vol}(E)$.

Nous commençons par établir la

Proposition 7.12. Soit $p \in S A_{f}$ et $B \subset \Omega(p)$ un petit voisinage de $p$.

Il existe des constantes $D_{1}, D_{2}>0$ t.q. pour tout borélien $E \subset B$ et pour tout $j \in \mathbf{N}$,

$$
\operatorname{Vol}\left(f^{j}(E)\right) \geq\left(D_{1} x(E)\right)^{D_{2} \rho(p)^{j}},
$$

où $x(E)$ est défini par la relation $-x(E) \log x(E)=\operatorname{Vol}(E)$.

Démonstration. La preuve est similaire à celle du lemme 3.3. Il nous faut estimer $\operatorname{Vol}\left\{\left|J f^{j}\right|^{2} \leq t\right\}$ pour $t \ll 1$ au voisinage de $p$. Pour cela on calcule explicitemement $J f$ à l'aide de la forme normale de $f$.

Classe 1:

$$
|J f|=\left|\lambda a_{1}\right||z|^{a_{1}+a_{2}-1} .
$$


Classe 2:

$$
|J f|=|z|^{a_{1}+a_{3}-1}|w|^{a_{2}+a_{4}-1} .
$$

Classe 3:

$$
|J f|=|\lambda \rho(p)||z|^{a_{1}(u+v)+b_{1}-1}|w|^{a_{2}(u+v)+b_{2}-1}(1+\psi)^{a_{1}-a_{2}},
$$

avec $\psi(z, w)=\lambda z^{b_{1}} w^{b_{2}}+P\left(z^{a_{1}} w^{a_{2}}\right)$.

On remplace alors dans les formules ci-dessus $f$ par son itéré $f^{j}$ et on en déduit une minoration de $|J f|^{j}$. Dans le cas des classes 1 et 2 , le calcul se fait simplement. Pour $f$ de la classe 3, on observe que

$$
\left.\left.f^{j+1}=\left(\left(z^{a_{1}} w^{a_{2}}\right)^{u \rho(p)^{j}}\left(1+\psi \circ f^{j}\right)\right)^{-a_{2}},\left(z^{a_{1}} w^{a_{2}}\right)^{v \rho(p)^{j}}\left(1+\psi \circ f^{j}\right)\right)^{a_{1}}\right) .
$$

On en déduit l'existence d'une constante $C>0$ t.q. pour tout $j \geq 0$

Classe 1:

avec $D=\left|\lambda a_{1}\right|$.

$$
|J f|^{j} \geq D^{j}|z|^{C \rho(p)^{j}}
$$

Classe 2:

$$
|J f|^{j} \geq|z w|^{C \rho(p)^{j}}
$$

Classe 3:

$$
|J f|^{j} \geq D^{j}|z|^{C \rho(p)^{j}},
$$

avec $D=\left|\lambda\left(a_{1} u+a_{2} v\right)\right|$.

On peut alors reprendre la démonstration du lemme 3.3 pour conclure.

Démonstration du théorème 7.11. La première assertion est une adaptation immédiate du théorème 3.11 de [Di 96] que nous laissons au lecteur.

Pour prouver la seconde et la troisième, on vérifie que pour $r \ll 1$ et $p \in S A_{f}$ fixés, et pour tout borélien $E \subset \Omega_{r}(p)$ et $j \in \mathbb{N}$ on a

$$
\operatorname{Vol}\left(f^{j}(E)\right) \geq\left(C_{1} x(E)\right)^{C_{2} \rho(p)^{j}} .
$$

On choisit $N \in \mathbb{N}$ assez grand pour que $f^{N}\left(\Omega_{r}(p)\right) \subset B$, où $B$ est un voisinage de $p$ sur lequel les estimations de la proposition 7.12 sont valides.

On peut de plus trouver $C>0$ t.q. pour tout borélien $E \subset \mathbb{P}^{1} \times \mathbb{P}^{1}$

$$
\operatorname{Vol}\left(f^{N}(E)\right) \geq(\operatorname{Vol}(E))^{C}
$$

(voir e.g. corollary 3.13 de [Di 96]). On a donc, pour $j \geq N$,

$$
\operatorname{Vol}\left(f^{j}(E)\right) \geq\left(\operatorname{Vol}\left(f^{j-N}(E)\right)^{C} \geq\left(D_{1} x(E)\right)^{C D_{2} \rho(p)^{j-N}},\right.
$$

ce qui achève la démonstration.

Démonstration du théorème 7.6. Soit $S \in \mathcal{T}\left(\mathbb{P}^{1} \times \mathbb{P}^{1}\right)$. Supposons que $\nu(p, S)>0$ pour un point $p \in \mathcal{E} x$. Quitte à changer $f$ en $f^{l}$, on peut supposer $f(p)=p$ et $f(V)=p$, où $V$ est le $\mathbb{R}$-cycle défini par le lemme 7.9. Soit $q \in V \backslash I_{f}$, on a

$$
\nu\left(f^{*} S, q\right) \geq \nu(S, f(q))=\nu(S, p)>0 .
$$

Ainsi $f^{*} S \geq c \cdot[V]$ pour une constante $c>0$. Mais alors $\forall j \in \mathbb{N}$,

$$
\frac{1}{\rho_{+}^{j}}\left(f^{j}\right)^{*} S \geq c \frac{1}{\rho_{+}^{j}}\left(f^{j-1}\right)^{*}[V]=\frac{c}{\rho_{+}}[V],
$$

donc $\rho_{+}^{-j}\left(f^{j}\right)^{*} S$ ne peut pas converger vers un multiple de $T^{+}$. 
Réciproquement, soit $S \in \mathcal{T}\left(\mathbb{P}^{1} \times \mathbb{P}^{1}\right)$ un courant cohomologue à $a_{S} \omega_{1}+$ $b_{S} \omega_{2}$ t.q. $\nu(p, S)=0$ pour tout point $p \in \mathcal{E} x$. Soit $\varphi=\mathcal{L}^{-1}(S)$ un potentiel normalisé de $S$ dans $\mathbb{C}^{2} \times \mathbb{C}^{2}$. La fonction psh $u_{j}:=\rho_{+}^{-j} \varphi \circ F^{j}$ est un potentiel de $\rho_{+}^{-j}\left(f^{j}\right)^{*}(S)$. On va prouver que $u_{j} \rightarrow c G^{+}$dans $L_{l o c}^{1}\left(\mathbb{C}^{4}\right)$ pour une constante $c>0$. La condition d'homogénéité sur $\varphi$ assure, $\forall(z, w) \in \mathbb{C}^{2} \times \mathbb{C}^{2}$,

$$
\varphi(z, w) \leq a_{S} \log |z|+b_{S} \log |w| .
$$

On en déduit $\forall j \geq 0, \forall(z, w) \in \mathbb{C}^{2} \times \mathbb{C}^{2}$,

$$
u_{j} \leq \rho_{+}^{-j}\left(a_{S} \log \left|P^{j}\right|+b_{S} \log \left|Q^{j}\right|\right) .
$$

Nous prouvons ci-après le

Lemme 7.13. Posons

$$
E_{\varepsilon}^{j}:=\left\{([z],[w]) \text { t.q. } u_{j}-\rho_{+}^{-j}\left(a_{S} \log \left|P^{j}\right|+b_{S} \log \left|Q^{j}\right|\right)<-\varepsilon\right\} \subset \mathbb{P}^{1} \times \mathbb{P}^{1} .
$$

Alors pour tout $\varepsilon>0, \lim _{j \rightarrow \infty} \operatorname{Vol}\left(E_{\varepsilon}^{j}\right)=0$.

On peut écrire $\rho_{+}^{-j} A^{j} \cdot{ }^{t}\left(a_{s}, b_{s}\right)=c^{t}\left(a^{+}, b^{+}\right)+{ }^{t}\left(a^{j}, b^{j}\right)$ avec $c>0$, où le dernier terme $\left(a^{j}, b^{j}\right)$ décroit exponentiellement vite vers zéro à la vitesse $\left(\rho_{-} / \rho_{+}\right)^{j}$. On a donc,

$$
\lim _{j \rightarrow \infty} \rho_{+}^{-j}\left(a_{S} \log \left|P^{j}\right|+b_{S} \log \left|Q^{j}\right|\right)=c G^{+} .
$$

Les $\left\{u_{j}\right\}$ forment une suite de fonctions psh localement uniformément majorées. On peut en extraire une sous-suite convergeant dans $L_{l o c}^{1}$ vers une fonction $u$ psh ou identiquement égale à $-\infty$. Le lemme précédent implique alors que $u=c G^{+}$presque partout, ce qui conclut la preuve.

Soit $S_{k}$ une suite de courants de masse uniformément bornée par 1 et dont le support évite un voisinage $V$ fixé de $\mathcal{E} x$. Alors en terme de classe de cohomologie, et pour des constantes $c_{k}>0$, on a $\rho_{+}^{-j}\left[\left(f^{j}\right)^{*} S_{k}\right] \rightarrow c_{k}\left[T^{+}\right]$ uniformément en $k$. D'autre part, une conséquence standard des inégalités de Harnack donne l'existence d'une constante $C>0$ t.q. les potentiels $\varphi_{k}:=\mathcal{L}^{-1}\left(S_{k}\right)$ satisfont $\varphi_{k} \geq c_{k} G^{+}-C$ dans $\pi^{-1}(V)$. En reprenant la démonstration précédente, on en déduit que $\rho_{+}^{-j} \varphi_{k} \circ F^{j} \rightarrow c_{k} G^{+}$uniformément en $k$.

Démonstration du lemme 7.13. Pour estimer $\operatorname{Vol}\left(E_{\varepsilon}^{j}\right)$, on remarque tout d'abord que $\forall \varepsilon>0, \forall j \geq 0$,

$$
f^{j}\left(E_{\varepsilon}^{j}\right) \subset E_{\varepsilon \rho_{+}^{j}}^{0},
$$

et on utilise les estimées volumiques précédentes (théorème 7.11).

Fixons $r>0$ arbitrairement petit, et notons

$$
F_{r}:=\left(\mathbb{P}^{1} \times \mathbb{P}^{1} \backslash \cup_{p \in S A_{f}} \Omega(p)\right) \bigcup_{p \in S A_{f} \backslash \mathcal{E} x} \Omega_{r}(p) .
$$

On va estimer tout d'abord $\operatorname{Vol}\left(E_{\varepsilon}^{j} \cap F_{r}\right)$. Il est classique (voir e.g. [Ki 00]) qu'il existe des constantes $D_{1}, D_{2}>0$ t.q. $\forall t>1$

$$
\operatorname{Vol}\left(E_{t}^{0}\right) \leq D_{1} \exp \left(-D_{2} t\right)
$$


Combinons les deux premières assertions du théorème 7.11. On peut trouver des constantes $C_{1}, C_{2}>0$ t.q. $\forall j \geq 0$,

$$
\begin{aligned}
\left(C_{1} x\left(E_{\varepsilon}^{j} \cap F_{r}\right)\right)^{C_{2} \rho_{0}^{j}} & \leq \operatorname{Vol}\left(f^{j}\left(E_{\varepsilon}^{j}\right) \cap F_{r}\right) \\
& \leq \operatorname{Vol}\left(E_{\varepsilon \rho_{+}^{j}}^{0}\right) \leq D_{1} \exp \left(-D_{2} \varepsilon \rho_{+}^{j}\right) .
\end{aligned}
$$

On en déduit que pour des constantes $C, C^{\prime}>0$

$$
x\left(E_{\varepsilon}^{j} \cap F_{r}\right) \leq C \exp \left(-C^{\prime}\left(\rho_{+} / \rho_{0}\right)^{j}\right) \underset{j \rightarrow \infty}{\longrightarrow} 0 .
$$

En notant $\Omega(\mathcal{E} x)=\cup_{p \in \mathcal{E} x} \Omega(p)$, et en faisant tendre $r$ vers 0 , on en déduit que $\lim _{j \rightarrow \infty} \operatorname{Vol}\left(E_{\varepsilon}^{j} \backslash \Omega(\mathcal{E} x)\right)=0$.

On va estimer maintenant $\operatorname{Vol}\left(E_{\varepsilon}^{j} \cap \Omega(\mathcal{E} x)\right)$. Pour cela on fixe $r>0$ assez petit et on étudie $\operatorname{Vol}\left(E_{\varepsilon}^{j} \cap \Omega_{r}(\mathcal{E} x)\right)$. On notera aussi $B_{\eta}(\mathcal{E} x)$ l'union des boules centrées sur $\mathcal{E} x$ et de rayon $\eta$. Par hypothèse, pour tout $p \in \mathcal{E} x$ on a $\nu(p, S)=0$. Le volume $\operatorname{Vol}\left(E_{t}^{0}\right)$ pour $t \gg 1$ est donc extrèmement petit au voisinage de $\mathcal{E} x$. Plus précisément (voir [Ki 00]), pour tout $R \gg 0$ fixé, il existe $\eta>0$ petit et $D_{3}>0$ t.q. pour tout $t \geq 1$,

$$
\text { Vol } \left.\left(E_{t}^{0} \cap B_{\eta}(\mathcal{E} x)\right)\right) \leq D_{3} \exp (-R t) .
$$

On peut appliquer la troisième assertion du théorème 7.11. Pour tout $j \in \mathbb{N}$ assez grand t.q. $f^{j} \Omega_{r}(\mathcal{E} x) \subset B_{\eta}(\mathcal{E} x)$ on obtient:

$$
\begin{aligned}
\left(C_{1} x\left(E_{\varepsilon}^{j} \cap \Omega_{r}(\mathcal{E} x)\right)\right)^{C_{2} \rho_{+}^{j}} & \leq \operatorname{Vol}\left(f^{j}\left(E_{\varepsilon}^{j}\right) \cap \Omega_{r}(\mathcal{E} x)\right) \\
& \leq \operatorname{Vol}\left(E_{\varepsilon \rho_{+}^{0}}^{0} \cap f^{j}\left(\Omega_{r}(\mathcal{E} x)\right)\right) \\
& \leq \operatorname{Vol}\left(E_{\varepsilon \rho_{+}^{0}}^{0} \cap B_{\eta}(\mathcal{E} x)\right) \leq D_{3} \exp \left(-R \varepsilon \rho_{+}^{j}\right) .
\end{aligned}
$$

Cela prouve que

$$
\varlimsup_{j \rightarrow \infty} x\left(E_{\varepsilon}^{j} \cap \Omega_{r}(\mathcal{E} x)\right) \leq C_{1}^{-1} \exp \left(-\varepsilon C_{2}^{-1} R\right)
$$

et on conclut en faisant tendre $R$ vers l'infini.

Le résultat suivant est une conséquence du théorème de convergence.

Proposition 7.14. Le courant de Green $T^{+}$est extrémal dans $\mathcal{T}\left(\mathbb{P}^{1} \times \mathbb{P}^{1}\right)$.

Démonstration. Soit $S \in \mathcal{T}\left(\mathbb{P}^{1} \times \mathbb{P}^{1}\right)$ tel que $S \leq T^{+}$. On veut montrer que $S$ est proportionnel à $T^{+}$. Considérons $S_{j}$ l'extension triviale de $\rho_{+}^{j}\left(f_{\mathbb{P}^{1} \times \mathbb{P}^{1} \backslash \mathcal{C}_{f^{-j}}}^{j}\right)^{*}(S)$ à travers $\mathcal{C}_{f^{-j}}$. Le courant $T^{+}$vérifie $\rho_{+}^{j}\left(f^{-j}\right)^{*} T^{+}=T^{+}$ dans $\mathbb{P}^{1} \times \mathbb{P}^{1} \backslash \mathcal{C}_{f^{-j}}$, on en déduit l'inégalité $S_{j} \leq T^{+}$valable dans tout $\mathbb{P}^{1} \times \mathbb{P}^{1}$ puisque $T^{+}$et $S_{j}$ ne chargent pas $\mathcal{C}_{f^{-j}}$.

Soit à présent $S_{j}^{\prime}=\left(f^{j}\right)^{*} S_{j} / \rho_{+}^{j}$. L'invariance de $T^{+}$donne $S_{j}^{\prime} \leq T^{+}$donc $S_{j}^{\prime}$ ne charge pas $\mathcal{C}_{f^{j}} \cup \mathcal{C}_{f^{-j}}$ (théorème 2.8). Mais on a clairement $S_{j}^{\prime}=S$ hors de $\mathcal{C}_{f^{j}} \cup \mathcal{C}_{f^{-j}}$, comme $S$ ne charge pas non plus les hypersurfaces, on en déduit que $S_{j}^{\prime}=S$ dans $\mathbb{P}^{1} \times \mathbb{P}^{1}$.

Notons $\theta_{1}$ et $\theta_{2}$ deux générateurs de $H^{1,1}\left(\mathbb{P}^{1} \times \mathbb{P}^{1}, \mathbb{R}\right)$ correspondants aux deux valeurs propres $\rho_{+}$et $\rho_{-}$de $A$. La classe de $S_{j}$ se décompose en 
$\left[S_{j}\right]=x_{j} \theta_{1}+y_{j} \theta_{2}$. On en déduit que

$$
[S]=\left[\frac{1}{\rho_{+}^{j}}\left(f^{j}\right)^{*} S_{j}\right]=x_{j} \theta_{1}+\left(\frac{\rho_{-}}{\rho_{+}}\right)^{j} y_{j} \theta_{2} .
$$

Puisque $f$ n'est ni linéaire, ni un produit croisé, on a $\left|\rho_{-}\right|<\rho_{+}$(cf lemme 2.1). Il s'ensuit que pour tout $j$, on a $y_{j}=0$ et $x_{j}=x$. Ainsi $S$ est cohomologue à $x T^{+}$avec $0 \leq x \leq 1$.

De $S_{j} \leq T^{+}$on déduit que $\operatorname{Supp} S_{j} \cap \bar{V}=\emptyset$, où $V$ désigne un voisinage assez petit de l'ensemble des points périodiques superattractifs de $f$. Le théorème 7.6 assure alors que $\left(f^{j}\right)^{*}\left(S_{j} / x\right) / \rho_{+}^{j}$ converge vers $T^{+}$, d'où $S=x T^{+}$.

\subsection{Applications birationnelles polynomiales dans $\mathbb{C}^{2}$.}

Soit $A \in \mathcal{M}_{2}(\mathbb{N})$ et $f \in \mathcal{M}_{A}$ une application birationnelle de $\mathbb{P}^{1} \times \mathbb{P}^{1}$. Il est naturel (cf proposition 1.5.2) de se restreindre aux applications telles que $I_{P} \cap I_{Q}=\emptyset$. Dans ce cas, la proposition 4.7 permet d'exprimer le degré topologique $d_{t}(f)=\alpha \delta+\beta \gamma=1$. On a donc $\beta \gamma=0$ ou $\alpha \delta=0$. Lorsque $\beta=0$ (le cas $\gamma=0$ se traite par symétrie), on obtient $\alpha=\delta=1$; le premier facteur est donc linéaire et la dynamique de ce produit croisé est assez simple à étudier. Dans la suite nous nous intéressons au cas $\alpha=0$ (le cas $\delta=0$ se traite similairement). On a $\beta=\gamma=1$ et on peut conjuguer $f$ linéairement pour se ramener à l'étude de

$$
f:([z],[w]) \mapsto\left([w],\left[C(w) z_{1}+D(w) z_{0}: A(w) z_{1}+B(w) z_{0}\right]\right),
$$

où $A, B, C, D$ sont des polynômes homogènes de degré $\delta$ en $w=\left(w_{0}, w_{1}\right)$.

Nous considérons ici le cas des applications qui sont polynomiales dans $\mathbb{C}^{2}$. Pour une application de la forme précédente, cela revient à imposer $C \equiv D \equiv 0$, i.e.

$$
f:(z, w) \in \mathbb{C}^{2} \mapsto(w, A(w) z+B(w)) \in \mathbb{C}^{2},
$$

avec $A, B \in \mathbb{C}[w]$ et $A \not \equiv 0$. Lorsque $A$ est un polynôme constant, on dira que $f$ est une application de Hénon.

Plus généralement, on introduit le semi-groupe suivant.

Définition 7.15. Soit $\mathcal{H}$ le semi-groupe des applications birationnelles polynomiales $f: \mathbb{C}^{2} \rightarrow \mathbb{C}^{2}$ s'écrivant sous la forme $f=h_{s} \circ \cdots \circ h_{1}$ avec

$$
h_{i}:(z, w) \mapsto\left(w, A_{i}(w) z+B_{i}(w)\right),
$$

où $A_{i}, B_{i} \in \mathbb{C}[w], A_{i} \not \equiv 0$, et $\delta_{i}:=\max \left\{1+\operatorname{deg}\left(A_{i}\right), \operatorname{deg}\left(B_{i}\right)\right\} \geq 2$.

Le théorème de Jung (voir théorème 2.6 de [F-M 89]) permet de montrer que tout automorphisme polynomial de $\mathbb{C}^{2}$ est conjugué par un automorphisme polynomial soit à une composée d'applications de Hénon, soit à une application élémentaire $(z, w) \mapsto(a z+b, c w+B(z))$ avec $a, c \in \mathbb{C}^{*}, b \in \mathbb{C}$, et $B \in \mathbb{C}[z]$, soit à un automorphisme affine.

Question. Toute application birationnelle polynomiale de $\mathbb{C}^{2}$ est-elle conjuguée par un automorphisme polynomial de $\mathbb{C}^{2}$ à l'une des applications suivantes?

1. un élément de $\mathcal{H}$; 
2. une application élémentaire de la forme $(z, w) \mapsto(a z+b, A(z) w+B(z))$ avec $a \in \mathbb{C}^{*}, b \in \mathbb{C}, A, B \in \mathbb{C}[z]$, et $A \not \equiv 0$.

3. un automorphisme affine.

La proposition suivante indique que tout élément de $\mathcal{H}$ se compactifie de manière algébriquement stable soit dans $\mathbb{P}^{2}$, soit dans $\mathbb{P}^{1} \times \mathbb{P}^{1}$.

Proposition 7.16. Soit $f=h_{1} \circ \cdots \circ h_{s} \in \mathcal{H}$ avec

$$
h_{i}:(z, w) \mapsto\left(w, A_{i}(w) z+B(w)\right),
$$

où $A_{i}, B_{i} \in \mathbb{C}[w]$ et $A_{i} \not \equiv 0$, et $\delta_{i}:=\max \left\{1+\operatorname{deg} A_{i}, \operatorname{deg} B_{i}\right\} \geq 2$. Notons $\delta$ le degré total de $f ; \rho_{+}$le rayon spectral de la matrice des degrés en $z, w$ de $f$ et $(a, b)$ le vecteur propre associé, à coefficients réels positifs normalisés par $a+b=1$.

(1) Si $\operatorname{deg} A_{s} \leq \operatorname{deg} B_{s}-1, f$ se compactifie en une application algébriquement stable de $\mathbb{P}^{2}$. L'hyperplan à l'infini $\{t=0\}$ est contracté par $f$ sur le point $q=[0: 1: 0]$ qui est superattractif. Soit $\Omega_{q} \subset \mathbb{C}^{2}$ son bassin d'attraction.

Le courant positif invariant $T^{+}:=\lim _{j \rightarrow \infty} \delta^{-j}\left(f^{j}\right)^{*} \omega_{F S}$ admet pour potentiel dans $\mathbb{C}^{2}$ la fonction $g^{+}:=\lim _{j \rightarrow \infty} \delta^{-j} \log ^{+}\left\|f^{j}\right\|$. Celle-ci est psh, continue, et $\left\{g^{+}>0\right\}=\Omega_{q}$.

(2) $S i \operatorname{deg} A_{s} \geq \operatorname{deg} B_{s}$, $f$ se compactifie en une application algébriquement stable de $\mathbb{P}^{1} \times \mathbb{P}^{1}$. Les deux droites à l'infini $\left\{z_{0} w_{0}=0\right\}$ sont contractées par $f^{2}$ sur le point $q=([0: 1],[0: 1])$ qui est superattractif. Soit $\Omega_{q} \subset \mathbb{C}^{2}$ son bassin d'attraction.

Le courant positif invariant $T^{+}:=\lim _{j \rightarrow \infty} \rho_{+}^{-j}\left(f^{j}\right)^{*}\left(a \omega_{1}+b \omega_{2}\right) a d-$ met pour potentiel dans $\mathbb{C}^{2}$ la fonction $g^{+}:=\lim _{j \rightarrow \infty} \rho_{+}^{-j} \log ^{+}\left\|f^{j}\right\|$. Celle-ci est psh, continue, et $\left\{g^{+}>0\right\}=\Omega_{q}$.

Comme $f$ est birationnelle, on peut également considérer $T^{-}$le courant invariant associé à son inverse. Le courant $T^{+}$admet un potentiel continu hors des points de $I_{\infty}^{+}=I_{f^{2}}$ qui sont en nombre fini, on peut donc définir le produit $T^{+} \wedge T^{-}$en raffinant la méthode de Bedford-Taylor [B-T 82] (voir [De 93] et [F-S 95b]).

Théorème 7.17. Soit $f \in \mathcal{H}$.

La mesure $\mu:=T^{+} \wedge T^{-} /\left\|T^{+} \wedge T^{-}\right\|$est une mesure de probabilité dans $\mathbb{C}^{2}$ qui ne charge pas l'infini et vérifie $f^{*} \mu=f_{*} \mu=\mu$.

La mesure $\mu$ est mélangeante.

Exemples 7.18. Nous nous intéressons ici au cas particulier $f(z, w)=$ $(w, A(w) z)$ avec $\operatorname{deg} A \geq 1)$. Considérons son extension méromorphe $\widetilde{f}$ dans $\mathbb{P}^{1} \times \mathbb{P}^{1}$. L'ensemble d'indétermination de $f$ est

$$
I^{+}=\{([1: 0],[0: 1]) ;([0: 1],[1, \zeta]) \text { où } A(\zeta)=0\}
$$

et on a

$$
I_{\infty}^{+}=I_{f^{2}}=I^{+} \cup\{([0: 1],[1: 0])\} .
$$

L'ensemble d'indétermination de l'inverse $\tilde{f}^{-1}$ est

$$
I^{-}=\{([0: 1],[0: 1]) ;([1: \zeta],[1: 0]) \text { où } A(\zeta)=0\} \text {. }
$$


L'ensemble $I_{\infty}^{-}$est constitué du point à l'infini $q=([0: 1],[0: 1])$ et de la réunion des orbites -sous l'action de $f$-des points $(\zeta, 0)$, où $\zeta \in A^{-1}(0)$. On obtient donc

$$
I_{\infty}^{-}=\left\{q ;\left(\lambda^{j} \zeta, 0\right),\left(0, \lambda^{j+1} \zeta\right) \text { avec } \zeta \in A^{-1}(0) \text { et } j \geq 0\right\},
$$

où on a posé $\lambda=A(0)$.

Lorsque $|\lambda|<1$, les points de $I_{f}$ sont envoyés par $f^{-1}$ sur le 2 -cycle $\{([1: 0],[0: 1]) ;([0: 1],[1: 0])\}$ qui est attractif pour $f^{-1}$. L'application $f$ est donc normale et le support de la mesure $\mu$ est compact dans $\mathbb{C}^{2}$.

Lorsque $|\lambda|=1$, on a $E^{+} \cap E^{-}=\emptyset$. Notons que la fonction de Green $g^{-}$ est alors discontinue sur les cercles $\{|z|=|\zeta|\} \times\{0\}$ et $\{0\} \times\{|w|=|\zeta|\}$ si $\lambda$ est d'argument irrationnel.

Lorsque $|\lambda|>1, E^{+} \cap E^{-}$est non vide, constitué du 2 -cycle $\{([0: 1],[1$ : $0]) ;([1: 0],[0: 1])\}$ qui est semi-répulsif pour $\tilde{f}^{-1}$ (de valeurs propres 0 et $\lambda)$. Nous pensons que le support de la mesure mélangeante $\mu$ n'est pas compact dans $\mathbb{C}^{2}$ lorsque $|\lambda| \geq 1$.

Démonstration de la proposition 7.16 Notons $f_{s}=\left(P_{s}, Q_{s}\right)=h_{1} \circ \cdots \circ$ $h_{s}$, nous allons démontrer par récurrence sur $s \geq 1$ que

1) si $\operatorname{deg} A_{s}<\operatorname{deg} B_{s}$, alors $\operatorname{deg}_{w} Q_{s}=\operatorname{deg} Q_{s}>\operatorname{deg} P_{s}=\operatorname{deg}_{w} P_{s}$;

2) si $\operatorname{deg} A_{s} \geq \operatorname{deg} B_{s}$, alors $\operatorname{deg}_{z} Q_{s}+\operatorname{deg}_{w} Q_{s}=\operatorname{deg} Q_{s}>\operatorname{deg} P_{s}=$ $\operatorname{deg}_{z} P_{s}+\operatorname{deg}_{w} P_{s}$.

L'assertion est claire pour $s=1$. Supposons la vérifiée au rang $s-1$ et écrivons les relations de récurrence

$$
\begin{aligned}
P_{s}(z, w) & =P_{s-1}\left(w, A_{s}(w) z+B_{s}(w)\right) \\
Q_{s}(z, w) & =Q_{s-1}\left(w, A_{s}(w) z+B_{s}(w)\right) .
\end{aligned}
$$

Il suffit alors d'examiner les quatres cas qui se présentent, selon que $f_{s-1}$ et $h_{s}$ sont de "type $\mathbb{P}^{2}$ " ou de "type $\mathbb{P}^{1} \times \mathbb{P}^{1}$ ".

Les assertions concernant le comportement de $f_{s}$ à l'infini en résultent, celles concernant le courant de Green découlent du théorème 5.2 lorsque $f$ admet une extension algébriquement stable dans $\mathbb{P}^{1} \times \mathbb{P}^{1}$, elles s'obtiennent de manière analogue -le contrôle de la croissance est plus simple à obtenirlorsque $f$ admet une extension algébriquement stable dans $\mathbb{P}^{2}$.

Démonstration du théorème 7.17. Supposons que $f$ se compactifie de manière algébriquement stable dans $\mathbb{P}^{1} \times \mathbb{P}^{1}$ (le cas $\mathbb{P}^{2}$ est analogue).

Le courant $T^{+}$admet un potentiel continu dans $\mathbb{C}^{2}$, comme de plus $T^{-}$ne charge pas les hypersurfaces, la mesure $\left.\mu\right|_{\mathbb{C}^{2}}$ ne charge pas les hypersurfaces (voir proposition 4.10). En particulier elle ne charge ni $\mathcal{C}^{+}$ni $\mathcal{C}^{-}$, ce qui assure $\left.f^{*} \mu\right|_{\mathbb{C}^{2}}=\left.f_{*} \mu\right|_{\mathbb{C}^{2}}=\left.\mu\right|_{\mathbb{C}^{2}}$.

Montrons à présent que $\mu$ ne charge pas les points d'indétermination de $f^{2}$, ce qui assurera que $\left.\mu\right|_{\mathbb{C}^{2}}$ est une mesure de probabilité puisque le support de $T^{+}$ne rencontre l'infini qu'aux points de $I_{\infty}^{+}=I_{f^{2}}$. Soit $p \in I_{\infty}^{+}$, on se place dans une carte locale avec $p=0$ et on note $G^{+}$un potentiel continu de $T^{+}$dans $B_{0} \backslash\{0\}$, où $B_{0}$ est une boule centrée en $p=0$. La fonction $G^{+}$est une fonction psh semi-exhaustive dans $B_{0}$ (voir definition $4.1 \mathrm{p} .137$ [De 93]) et la masse de $\mu$ en $p=0$ est un multiple du nombre de Lelong $\nu\left(T^{-}, G^{+}\right)$de $T^{-}$par rapport à la fonction $G^{+}$(definition 4.2 p.137 [De 
93]). La proposition 5.6 assure

$$
\limsup _{z \rightarrow 0} \frac{G^{+}(z)}{\log |z|} \leq c<+\infty .
$$

Le théorème de comparaison des nombres de Lelong généralisés de Demailly (First comparison theorem p.148 [De 93]) donne alors $\nu\left(T^{-}, G^{+}\right) \leq$ $c \cdot \nu\left(T^{-}, 0\right)$. Comme $f$ est algébriquement stable, on a $I_{\infty}^{+} \cap I_{\infty}^{-}=\emptyset$ (proposition 7.4), d'où $\nu\left(T^{-}, 0\right)=0$ (proposition 7.5). Il s'ensuit que $\mu$ ne charge pas le point $p=0$.

Nous en déduisons à présent le mélange de $\mu$ en reprenant la démonstration proposée par Sibony dans le cas des automorphismes polynomiaux réguliers de $\mathbb{C}^{k}$ (théorème 7.1 p.52 [Si 99]). Nous renvoyons à [Wa 82] pour les définitions concernant le mélange (fort). Si $\psi$ et $\chi$ sont des fonctions test, il s'agit de montrer que

$$
\int \psi\left(f^{-j}\right) \chi d \mu \rightarrow\left(\int \psi d \mu\right)\left(\int \chi d \mu\right)
$$

Puisque $\mu$ ne charge pas les points de $I_{\infty}^{+}$, ni les droites à l'infini, on peut supposer que les supports de $\psi$ et $\chi$ sont inclus dans $\mathbb{C}^{2}$. Enfin on ne perd rien à supposer que $0 \leq \psi, \chi \leq 1$. Considérons $R_{j}:=\left(f^{-j}\right)^{*}\left(\psi T^{-}\right) / \rho_{+}^{j}$. La proposition 7.19 qui suit montre que $R_{j}$ converge vers $c_{\psi} \cdot T^{-}$avec $c_{\psi}=$ $\int \psi d \mu$ et $d R_{j}, d d^{c} R_{j}$ convergent vers 0 en masse. Il s'ensuit que $\psi\left(f^{-j}\right) T^{+} \wedge$ $T^{-}$converge vers $c_{\psi} T^{+} \wedge T^{-}$. Soit en effet $\theta$ une fonction test à support dans une boule de $\mathbb{C}^{2}$, on note $G^{+}$un potentiel continu de $T^{+}$dans cette boule. Alors

$$
\begin{aligned}
& <\psi\left(f^{-j}\right) T^{-} \wedge T^{+}, \theta>=<\frac{1}{\rho_{+}^{j}}\left(f^{-j}\right)^{*}\left(\psi T^{-}\right) \wedge T^{+}, \theta> \\
& =<d d^{c}\left(\frac{1}{\rho_{+}^{j}}\left(f^{-j}\right)^{*}\left(\psi T^{-}\right) \theta\right), G^{+}> \\
& =<R_{j} \wedge d d^{c} \theta, G^{+}>+<\theta d d^{c} R_{j}, G^{+}>+2<d R_{j} \wedge d^{c} \theta, G^{+}>.
\end{aligned}
$$

Le premier terme converge vers $\left\langle c_{\psi} \cdot T^{-} \wedge d d^{c} \theta, G^{+}\right\rangle=\left\langle c_{\psi} \cdot T^{+} \wedge T^{-}, \theta\right\rangle$ puisque $G^{+}$est continue au voisinage du support de $\theta$ et les deux derniers convergent vers 0 puisque $\left\|d R_{j}\right\|$ et $\left\|d d^{c} R_{j}\right\|$ convergent vers 0 . Ainsi

$$
\int \psi\left(f^{j}\right) \chi d \mu=<\psi\left(f^{j}\right) T^{+} \wedge T^{-}, \frac{\chi}{\left\|T^{+} \wedge T^{-}\right\|}>\longrightarrow<\mu, \psi><\mu, \chi>.
$$

Proposition 7.19. Soit $f \in \mathcal{H}$ et $\psi$ une fonction test.

La suite de courants

$$
R_{j}:=\frac{1}{\rho_{+}^{j}}\left(f^{-j}\right)^{*}\left(\psi T^{-}\right)=\left(\psi \circ f^{-j}\right) T^{-}
$$

converge vers $c_{\psi} \cdot T^{-}$, où $c_{\psi}=<\mu, \psi>=\int \psi T^{+} \wedge T^{-} /\left\|T^{+} \wedge T^{-}\right\|$. De plus $d R_{j}$ et $d d^{c} R_{j}$ convergent vers 0 en masse. 
Démonstration. La démonstration est une adaptation immédiate de celle de Sibony, proposition II.6.1 [Si 99].

\section{REFERENCES}

Be 83 A.BEAUVILLE: Complex algebraic surfaces, Cambridge univ. press, 1983.

Br 97 J.-Y.BRIEND: Exposants de Lyapunov et points péridodiques d'endomorphismes holomorphes de $\mathbb{C P}^{k}$. Thèse, Toulouse, 1997.

Br-D 99 J.-Y.BRIEND \& D.DUVAL: Exposants de Liapunoff et distribution des points périodiques d'un endomorphisme de $\mathbb{P}^{k}$. Acta Math. 182 (1999), no. 2, 143-157.

B-S 91 E.BEDFORD \& J.SMILLIE: Polynomial diffeomorphisms of $\mathbb{C}^{2}$ : Currents, equilibrium measure and hyperbolicity. Invent. Math. 103 (1991), 69-99.

B-S 92 E.BEDFORD \& J.SMILLIE: Polynomial diffeomorphisms of $\mathbb{C}^{2}$ III: Ergodicity, exponents and entropy of the equilibrium measure. Math. Ann. 294 (1992), 395-420.

B-T 82 E.BEDFORD \& B.-A.TAYLOR: A new capacity for plurisubharmonic functions. Acta Math. 149 (1982), 1-40.

De 93 J.-P.DEMAILLY: Monge-Ampère Operators, Lelong numbers and intersection theory, in Complex Analysis and Geometry. Ed. A.Ancona and A.Silva. Plenum Press (1993), 115-193.

Di 96 J.DILLER: Dynamics of birational maps of $\mathbb{P}^{2}$. Indiana Univ. Math. J. 45 (1996), 721-772.

Di 98 J.DILLER: dynamics of birational maps of $\mathbb{P}^{2}$ II: limits of non-closed currents and the invariant measure. Preprint (1998).

Fa 99 C.FAVRE: Pull-back and Lelong number of currents. Bull. Soc. Math. France 127 (1999), no. 3, 445-458.

Fa 00 C.FAVRE: Classification of two-dimensional rigid germs and Kato surfaces. J. Math. Pures Appl. (9) 79 (2000), no. 5, 475-514.

F 91 S.FRIEDLAND: Entropy of polynomial and rational maps. Ann. of Math. 133 (1991), 359-368.

F-M 89 S.FRIEDLAND \& J.MILNOR: Dynamical properties of plane automorphisms. Erg. Th. and Dynam. Syst. 9 (1989). 67-99.

F-S 92 J.-E.FORNAESS \& N.SIBONY: Complex Henon mappings in $\mathbb{C}^{2}$ and Fatou Bieberbach domains. Duke Math. J. 65 (1992), 345-380.

F-S 94 J.-E.FORNAESS \& N.SIBONY: Complex dynamics in higher dimension I. Astérisque 222 (1994), 201-213.

F-S 95 J.-E.FORNAESS \& N.SIBONY: Complex dynamics in higher dimension II. In Annals of Mathematics Studies 137. Princeton university press (1995), $135-182$.

F-S 95b J.-E.FORNAESS \& N.SIBONY: Oka's inequality for currents, Math. Ann. 301 (1995), 399-419.

G-97 V.GUEDJ: Representation theorems for positive closed $(1,1)$-currents on flag manifolds of $G L_{m}(\mathbb{C})$, preprint (1997).

G-S 99 V.GUEDJ \& N.SIBONY: Dynamics of polynomial automorphisms of $\mathbb{C}^{k}$, preprint.

He 96 S.-M.HEINEMANN: Julia sets for holomorphic endomorphisms of $\mathbb{C}^{n}$. Ergodic Theory Dyn. Syst. 16 (1996), 1275-1296.

Jo 99 M.JONSSON: Dynamics of polynomial skew products on $\mathbb{C}^{2}$. Math. Ann. 314 (1999), no. 3, 403-447.

Jo 00 M.JONSSON: Ergodic properties of fibered rational maps, Ark. Mat. 38 (2000), no. 2, 281-317.

K-H 95 A.KATOK \& HASSELBLATT: Introduction to the modern theory of dynamical systems. Cambridge Univ. Press (1995).

Ki 00 C.KISELMAN: Ensembles de sous-niveau et images inverses des fonctions plurisousharmoniques. Bull. Sci. Math. 124 (2000), no. 1, 75-92 
P-S 91 T.PETERNELL \& M.SCHNEIDER: Compactifications of $\mathbb{C}^{n}$ : A Survey. In Proc. of Symp. in Pure Mathematics vol. 52, part 2. Several Complex Variables and Geometry (1991), 455-466.

P 85 F.PRZYTYCKI: Hausdorff dimension of harmonic measure on the boundary of an attractive basin for a holomorphic map. Inv. Math. 80 (1985), 161-179.

R-S 97 A.RUSSAKOVSKII \& B.SHIFFMAN: Value distribution for sequences of rational mappings and complex dynamics. Indiana Univ. Math. J. 46 (1997), 897-932.

Se 97 O.SESTER: Etude dynamique des polynômes fibrés. Thèse de doctorat, Université Paris-sud (1997).

Si 85 N.SIBONY: Quelques problèmes de prolongement de courants en Analyse Complexe. Duke Math. J. 52 (1985),157-197.

Si 99 N.SIBONY: Dynamique des applications rationnelles de $\mathbb{P}^{k}$. Panorama et Syntheses, 8, Soc. Math. France, Paris, 1999.

Sk 82 H.SKODA: Prolongement des courants positifs fermés de masse finie. Inv. Math. 66 (1982), 361-376.

S 74 Y.-T.SIU: Analyticity of sets associated to Lelong numbers and extension of closed positive currents. Invent. Math. 27 (1974), 53-156.

Wa 82 P.WALTERS: An introduction to ergodic theory. Springer Verlag 1982.

Y 95 L.-S.YOUNG: Ergodic theory of differentiable dynamical systems. In B. Branner, P.Hjorth ed., Real and complex dynamical systems. Kluwer Academic Publishers (1995), 293-336.

Mathematiques, Bat. 425, Université de Paris-Sud, F-94105 Orsay Cedex, FRANCE

E-mail address: Charles.Favre@math.u-psud.fr

Université Paris 7, UFR de Mathématiques, Equipe Géométrie et Dynamique, Case 7012, 2, Place Jussieu, 75251 Paris, Cedex 05

E-mail address: favre@math.jussieu.fr

Laboratoire Emile Picard, Université Paul Sabatier, 118 route de NarBonne, 31062 Toulouse Cedex 4, France

E-mail address: guedj@picard.ups-tlse.fr 